\title{
A UNIFIED APPROACH ON SPRINGER FIBERS IN THE HOOK, TWO-ROW AND TWO-COLUMN CASES
}

\author{
LUCAS FRESSE
}

\begin{abstract}
We consider the Springer fiber over a nilpotent endomorphism. Fix a Jordan basis and consider the standard torus relative to this. We deal with the problem to describe the flags fixed by the torus which belong to a given component of the Springer fiber. We solve the problem in the hook, two-row and two-column cases. We provide two main characterizations which are common to the three cases, and which involve dominance relations between Young diagrams and combinatorial algorithms. Then, for these three cases, we deduce topological properties of the components and their intersections.
\end{abstract}

\section{INTRODUCTION}

Let $G$ be a connected reductive algebraic group over $\mathbb{C}$ and let $\mathfrak{g}$ be its Lie algebra. The set $\mathcal{B}$ of the Borel subalgebras $\mathfrak{b} \subset \mathfrak{g}$ is a projective algebraic variety, called the flag variety. For a nilpotent element $u \in \mathfrak{g}$, the set

$$
\mathcal{B}_{u}=\{\mathfrak{b} \in \mathcal{B}: u \in \mathfrak{b}\}
$$

is a closed subvariety of $\mathcal{B}$. The variety $\mathcal{B}_{u}$ is called a Springer fiber since it is the fiber over $u$ of the Springer resolution $\mathcal{X} \rightarrow \mathcal{N},(\mathfrak{b}, u) \mapsto u$, where $\mathcal{N} \subset \mathfrak{g}$ is the subset of nilpotent elements and $\mathcal{X}=\{(\mathfrak{b}, u) \in \mathcal{B} \times \mathcal{N}: u \in \mathfrak{b}\}$ (see [15]).

The study of Springer fibers involves different fields as algebraic geometry, representation theory and combinatorics. The origin of the study dates back to the geometric realization, due to T.A. Springer, of the irreducible representations of the Weyl groups in the cohomology of Springer fibers (see [16]). D. Kazhdan and G. Lusztig gave a topological construction of Springer representations (see 7]) and conjectured a link between the configuration of the irreducible components of Springer fibers and the construction of bases for the representations of the Hecke algebra (see [6. §6.3]). These have been strong motivations which have made Springer fibers be an important topic of study in modern algebra.

However, up to now, few questions have been solved. Even for the type $A$, advances have been done only in few particular cases. One of the major difficulties seems to be that the geometry of $\mathcal{B}_{u}$ strongly depends on the Jordan form of $u$, and the study in each case is very specific.

Throughout this article, we study the Springer fibers for $G=G L\left(\mathbb{C}^{n}\right)$ : we set $V=\mathbb{C}^{n}$, and $u: V \rightarrow V$ is a nilpotent endomorphism. We identify $\mathcal{B}$ with the variety of complete flags, i.e. chains of subspaces $\left(0=V_{0} \subset V_{1} \subset \ldots \subset V_{n}=V\right)$ with $\operatorname{dim} V_{i}=i$ for every $i=0, \ldots, n$. We identify $\mathcal{B}_{u}$ with the closed subvariety of complete flags $\left(V_{0}, \ldots, V_{n}\right) \in \mathcal{B}$ which are stable by $u$, i.e. $u\left(V_{i}\right) \subset V_{i}$ for every $i=0, \ldots, n$. 
It is known from N. Spaltenstein [13, [14] and R. Steinberg [17] that the irreducible components $\mathcal{K}^{T} \subset \mathcal{B}_{u}$ are parameterized by a set of standard tableaux $T$. We recall Spaltenstein's construction in 1.1 .

We study the components from the point of view of a set of special flags $F_{\tau} \in \mathcal{B}_{u}$ which are parameterized by a set of row-standard tableaux $\tau$, and depend on the initial choice of a Jordan basis. Formally, they are the elements in $\mathcal{B}_{u}$ which are fixed by the standard torus relative to the basis, for its linear action on flags.

The main part of this article concerns the problem to determine whether the flag $F_{\tau}$ lies in a given component $\mathcal{K}^{T}$, and to interpret this problem into a combinatorial one involving the pair $(\tau, T)$. We formulate the problem in an intrinsic way in the sequel of the present section.

First, we establish necessary or sufficient criteria in the general case (sections 2 , 3 and (4).

Next, we give the full answer in three particular cases: the hook, two-row and two-column cases. We provide two main characterizations, which are common to the three cases. The first one involves a series of dominance relations between Young diagrams (section 5). The second one relies on an algorithm, whose aim is to construct the tableau $\tau$ according to certain rules depending on $(\tau, T)$ : if the construction succeeds, then the pair $(\tau, T)$ is said to be constructible, and we show that $F_{\tau} \in \mathcal{K}^{T}$ exactly in this case (section 6).

Finally, in these three cases, we provide a connection between the combinatorics involved in our criteria and the existing combinatorics involved in the previous studies of the components of the Springer fibers in [5], 10, 11, 12, 18] (section 7), and we deduce topological properties of the components and their intersections, especially the intersections in codimension one, which play a crucial role in Kazhdan-Lusztig's conjecture (section 8). Especially we provide a new characterization of the pairs of components intersecting in codimension one in the two-row case (Theorem 29). In the three cases, we give a simple necessary condition for having an intersection in codimension one (Theorem 41).

1.1. Spaltenstein's construction of the components of $\mathcal{B}_{u}$. Recall that a Young diagram is a set of empty boxes displayed along left-justified rows, whose length decreases to the bottom. If $Y$ is a Young diagram with $n$ boxes, a standard tableau of shape $Y$ is a numbering of $Y$ by $1, \ldots, n$ such that numbers in the rows increase to the right and numbers in the columns increase to the bottom.

Up to isomorphism, the Springer fiber $\mathcal{B}_{u}$ only depends on the Jordan form of $u$, which can be represented by a Young diagram: let $\lambda_{1} \geq \ldots \geq \lambda_{r}$ be the sizes of the Jordan blocks of $u$, then we denote by $Y(u)$ the Young diagram of rows of lengths $\lambda_{1}, \ldots, \lambda_{r}$. Let $\lambda_{1}^{*} \geq \ldots \geq \lambda_{s}^{*}$ be the lengths of the columns of $Y(u)$. We know that (see [14, §II.5.5])

$$
\operatorname{dim} \mathcal{B}_{u}=\sum_{j=1}^{s} \frac{1}{2} \lambda_{j}^{*}\left(\lambda_{j}^{*}-1\right) .
$$

Let us recall from [14 that the irreducible components of $\mathcal{B}_{u}$ are parameterized by the standard tableaux of shape $Y(u)$. For $T$ standard, the shape of the subtableau $T[1, \ldots, i]$ of entries $1, \ldots, i$ is a subdiagram $Y_{i}^{T} \subset Y(u)$. In this manner we associate to $T$ an increasing sequence of subdiagrams $Y_{1}^{T} \subset \ldots \subset Y_{n}^{T}=Y(u)$. On the other hand, let $F=\left(V_{0}, \ldots, V_{n}\right) \in \mathcal{B}_{u}$. The restriction of $u$ to $V_{i}$ is a 
nilpotent endomorphism of $V_{i}$, whose Jordan form is represented by the Young diagram $Y\left(u_{\mid V_{i}}\right) \subset Y(u)$. Thus, we associate to the flag $F$ an increasing sequence of subdiagrams $Y\left(u_{\mid V_{1}}\right) \subset \ldots \subset Y\left(u_{\mid V_{n}}\right)=Y(u)$. Then, we define

$$
\mathcal{B}_{u}^{T}=\left\{F=\left(V_{0}, \ldots, V_{n}\right) \in \mathcal{B}_{u}: Y\left(u_{\mid V_{i}}\right)=Y_{i}^{T} \forall i=1, \ldots, n\right\} .
$$

By [14, §II.5], the $\mathcal{B}_{u}^{T}$ 's form a partition of $\mathcal{B}_{u}$ into locally closed, irreducible, nonsingular subsets, and $\operatorname{dim} \mathcal{B}_{u}^{T}=\operatorname{dim} \mathcal{B}_{u}$ for every $T$. Set

$$
\mathcal{K}^{T}=\overline{\mathcal{B}_{u}^{T}}
$$

the Zariski closure. It follows that $\mathcal{K}^{T}$ is an irreducible component of the variety $\mathcal{B}_{u}$, and each component of $\mathcal{B}_{u}$ is obtained in that way. In particular $\operatorname{dim} \mathcal{K}^{T}=\operatorname{dim} \mathcal{B}_{u}$ for every $T$.

1.2. Flags $F_{\tau} \in \mathcal{B}_{u}$ and statement of the problem. If $Y$ is a Young diagram with $n$ boxes, then we call row-standard tableau of shape $Y$ a numbering of $Y$ by $1, . ., n$ such that numbers in the rows increase to the right.

We parameterize some special elements $F_{\tau} \in \mathcal{B}_{u}$ with the row-standard tableaux of shape $Y(u)$. To do this, we fix a Jordan basis of $u$. Since the lengths of the rows of $Y(u)$ correspond to the sizes of the Jordan blocks of $u$, we can index the basis $\left(e_{x}\right)_{x \in Y(u)}$ on the boxes of $Y(u)$, in such a way that the following is true:

- $u\left(e_{x}\right)=0$ when $x$ lies in the first column of $Y(u)$,

- $u\left(e_{x}\right)=e_{x^{\prime}}$, where $x^{\prime}$ is the box just on the left of $x$, otherwise.

Let $\tau$ be a row-standard tableau of shape $Y(u)$. For $i=1, \ldots, n$, the numbers $1, \ldots, i$ correspond to boxes $x_{1}, \ldots, x_{i} \in Y(u)$ according to the numbering of $Y(u)$ given by $\tau$. Then we set $V_{i}=\left\langle e_{x_{1}}, \ldots, e_{x_{i}}\right\rangle$, and we define

$$
F_{\tau}=\left(V_{0}=0, V_{1}, \ldots, V_{n}\right) \text {. }
$$

As $\tau$ is row-increasing, it is clear by construction that $F_{\tau} \in \mathcal{B}_{u}$. Moreover, it is easy to see that the flags $F_{\tau}$, for $\tau$ running over the set of row-standard tableaux of shape $Y(u)$, are all the elements of $\mathcal{B}_{u}$ which are fixed by the action on flags of the torus of the automorphisms diagonal in the Jordan basis.

In this article, we deal with the following problem: let $\tau$ be a row-standard tableau and let $T$ be a standard tableau of shape $Y(u)$, when does the flag $F_{\tau}$ belong to the component $\mathcal{K}^{T}$ ?

1.3. Orbits $\mathcal{Z}_{\tau} \subset \mathcal{B}_{u}$ and an intrinsic statement of the problem. The definition of the flags $F_{\tau}$ depends on the choice of a Jordan basis of $u$. We relate these flags to a notion of special orbits which is intrinsic.

Let $\tau$ be a row-standard tableau of shape $Y(u)$. A basis $\left(e_{i}\right)_{i=1, \ldots, n}$ of $V$ is said to be a $\tau$-basis if we have

- $u\left(e_{i}\right)=0$ when $i$ is in the first column of $\tau$,

- $u\left(e_{i}\right)=e_{j}$, where $j$ is the number just on the left of $i$ in $\tau$, otherwise.

For $\underline{e}=\left(e_{i}\right)_{i=1, \ldots, n}$ a $\tau$-basis, we denote by $F(\underline{e})$ the flag defined by

$$
F(\underline{e})=\left(V_{0}=0, V_{1}, \ldots, V_{n}\right)
$$

with $V_{i}=\left\langle e_{1}, \ldots, e_{i}\right\rangle$. It is clear that $F(\underline{e}) \in \mathcal{B}_{u}$. Finally we denote by $\mathcal{Z}_{\tau}$ the set of flags $F(\underline{e})$ for $\underline{e}$ running over the set of $\tau$-bases.

Let $Z(u)=\{g \in G L(V): g u=u g\}$. The group $Z(u)$ is connected, and its natural action on flags leaves $\mathcal{B}_{u}$ and every component of $\mathcal{B}_{u}$ stable. Observe that 
$Z(u)$ acts transitively on the set of $\tau$-bases. It follows that $\mathcal{Z}_{\tau}$ is a $Z(u)$-orbit of $\mathcal{B}_{u}$. In addition, the flag $F_{\tau}$ belongs to the set $\mathcal{Z}_{\tau}$. Indeed, if we write $e_{i}:=e_{x_{i}}$ for every $i=1, \ldots, n$, where $e_{x_{i}}$ is the basic vector involved in the definition of $F_{\tau}$, then we get a $\tau$-basis $\underline{e}=\left(e_{1}, \ldots, e_{n}\right)$ such that $F_{\tau}=F(\underline{e})$. Thus, we have shown:

Proposition 1. (a) The subset $\mathcal{Z}_{\tau} \subset \mathcal{B}_{u}$ is the $Z(u)$-orbit of the flag $F_{\tau}$.

(b) Let $\mathcal{K}^{T} \subset \mathcal{B}_{u}$ be an irreducible component. The following conditions are equivalent: (i) $\mathcal{Z}_{\tau} \cap \mathcal{K}^{T} \neq \emptyset$; (ii) $\mathcal{Z}_{\tau} \subset \mathcal{K}^{T}$; (iii) $F_{\tau} \in \mathcal{K}^{T}$.

Let us introduce an equivalence relation on row-standard tableaux: we write $\tau \equiv \tau^{\prime}$ if $\tau^{\prime}$ can be obtained from $\tau$ by switching one or several couples of rows of same length. Observe that, for $\tau \equiv \tau^{\prime}, \tau$-bases coincide with $\tau^{\prime}$-bases, which implies $\mathcal{Z}_{\tau}=\mathcal{Z}_{\tau^{\prime}}$. On the other hand, suppose that $i$ is on the left of $j$ in $\tau$, and let $F=\left(V_{0}, \ldots, V_{n}\right) \in \mathcal{Z}_{\tau}$. Then $i$ is minimal such that $u\left(V_{j}\right) \subset V_{i}+u\left(V_{j-1}\right)$. It follows that $\mathcal{Z}_{\tau} \cap \mathcal{Z}_{\tau^{\prime}}=\emptyset$ whenever $\tau \not \equiv \tau^{\prime}$. We have then proved:

Proposition 2. We have $\mathcal{Z}_{\tau}=\mathcal{Z}_{\tau^{\prime}}$ if and only if $\tau \equiv \tau^{\prime}$.

Remark 1. In general, the $\mathcal{Z}_{\tau}$ 's are not all the $Z(u)$-orbits of $\mathcal{B}_{u}$. Suppose for example $Y(u)=\square$, let $\left(e, e^{\prime}, e^{\prime \prime}, f\right)$ with $u\left(e^{\prime \prime}\right)=e^{\prime}, u\left(e^{\prime}\right)=e, u(e)=u(f)=0$ be a Jordan basis, then the flag $F=\left(0 \subset\langle e\rangle \subset\left\langle e, e^{\prime}+f\right\rangle \subset\left\langle e, e^{\prime}, f\right\rangle \subset V\right)$ does not belong to any $\mathcal{Z}_{\tau}$. Nevertheless, the $\mathcal{Z}_{\tau}$ 's are all the $Z(u)$-orbits of $\mathcal{B}_{u}$ when the diagram $Y(u)$ has two columns (see [3]).

Let $(\tau, T)$ be a pair formed by a row-standard tableau and a standard tableau of shape $Y(u)$. Observe that the property that the flag $F_{\tau}$ belongs to the component $\mathcal{K}^{T}$ (or equivalently that the orbit $\mathcal{Z}_{\tau}$ lies in $\mathcal{K}^{T}$ ) does not depend on $u$. Indeed, if $u^{\prime}: V^{\prime} \rightarrow V^{\prime}$ is a nilpotent endomorphism of same Jordan form as $u$, then we have $u^{\prime}=g u g^{-1}$ for some linear isomorphism $g: V \rightarrow V^{\prime}$, and $g$ induces an isomorphism $\mathcal{B}_{u} \rightarrow \mathcal{B}_{u^{\prime}}$ which sends the component of $\mathcal{B}_{u}$ associated to $T$ to the component of $\mathcal{B}_{u^{\prime}}$ associated to $T$ on one hand, and makes correspond the $\tau$-bases of $V$ with the $\tau$-bases of $V^{\prime}$ on the other hand.

Let $\operatorname{sh}(\tau)$ and $\operatorname{sh}(T)$ denote the shapes of $\tau$ row-standard or $T$ standard, and let $|\tau|$ (resp. $|T|$ ) denote the number of boxes in $\tau$ (resp. $T$ ). Define $\mathbf{Y}$ to be the set of pairs $(\tau, T)$ formed by $\tau$ row-standard and $T$ standard, with $\operatorname{sh}(\tau)=\operatorname{sh}(T)$. For $(\tau, T) \in \mathbf{Y}$, we write $\tau \in T$ if the flag $F_{\tau}$ belongs to the component $\mathcal{K}^{T}$ in the Springer fiber $\mathcal{B}_{u}$ over any $u$ nilpotent such that $Y(u)=\operatorname{sh}(T)$. Define $\mathbf{K}$ to be the subset of pairs $(\tau, T) \in \mathbf{Y}$ such that $\tau \in T$.

Thus, the main purpose of this article is to study the combinatorial properties of the set $\mathbf{K}$.

1.4. The hook, two-row and two-column cases. A Young diagram $Y$ is said to be of hook type if it contains at most one row of length $\geq 2$. It is said to be of two-row type if it contains at most two rows. It is said to be of two-column type if it contains at most two columns. A nilpotent endomorphism $u \in \operatorname{End}(V)$ is said to be of hook (resp. two-row) (resp. two-column) type, according to its Young diagram $Y(u)$. The most often in this article, we specialize in these three cases. One common specificity, which makes the study easier in these cases, is that the dominance order on diagrams is here linear. Moreover, we point out some special properties held by Springer fibers in these three cases (cf. Proposition 12 and the remarks in section 8.4). 
Up to now, the description of the components of Springer fibers has essentially been confined to these three cases. For instance, in each case, an equational characterization of the components has been provided (see [2], [5], 18]) and sometimes we shall rely on these previous studies. Nevertheless, these equations are a bit cumbersome and make each case be very specific. The aim in studying the components under the point of view of the belonging of the special orbits $\mathcal{Z}_{\tau}$, is to get more convenient and unified combinatorial descriptions in the three cases.

1.5. Notation. The notation of the previous subsections is kept. Throughout this article, the flags are written $\left(V_{0}, \ldots, V_{n}\right)$ or $\left(V_{0} \subset \ldots \subset V_{n}\right)$ or $F$. The standard tableaux are usually written $T, T^{\prime}, S, \ldots$ The row-standard tableaux are written $\tau, \tau^{\prime}, \ldots$ We will write interchangeably $\tau \in T$ or $(\tau, T) \in \mathbf{K}$. We denote by \# $A$ the number of elements in a finite set $A$. The reader can find an index of notation at the end of this article.

\section{Some basic properties of the Set $\mathbf{K}$, And CONNECtion to the PROBLEM OF INTERSECTIONS OF COMPONENTS}

First in this section, we introduce the standardization $\operatorname{st}(\tau)$ of a row-standard tableau $\tau$ (i.e. the standard tableau obtained by putting the entries in each column of $\tau$ in right order). Then, we point out quite natural properties involving $\operatorname{st}(\tau)$, for instance that the flag $F_{\tau}$ belongs to the set $\mathcal{B}_{u}^{\text {st }(\tau)}$, and thus to the component $\mathcal{K}^{\text {st }(\tau)}$ (hence $\tau \in \operatorname{st}(\tau)$, or equivalently $(\tau, \operatorname{st}(\tau)) \in \mathbf{K})$. Of course, it does not solve our initial problem, because there can also exist $T \neq \operatorname{st}(\tau)$ such that $\tau \in T$. We prove also: $\tau \in T \Rightarrow \operatorname{st}(\tau) \in T$. Finally, we show that two given components $\mathcal{K}^{T}, \mathcal{K}^{T^{\prime}}$ have a nonempty intersection if and only if there is a common $\tau$ which is standard such that $\tau \in T$ and $\tau \in T^{\prime}$.

2.1. Standardization of a row-standard tableau. Let $\tau$ be a row-standard tableau. Arrange the entries in each column of $\tau$ in increasing order to the bottom. Then, we see that the numbers in the rows of the tableau so-obtained remain increasing to the right. In other words, this tableau is standard. We denote it by $\operatorname{st}(\tau)$. For example

$$
\tau=\begin{array}{|l|l|l}
\hline 3 & 4 & 8 \\
\hline 1 & 6 & 7 \\
\hline 2 & 5 &
\end{array} \quad \operatorname{st}(\tau)=\begin{array}{|l|l|l|}
\hline 1 & 4 & 7 \\
\hline 2 & 5 & 8 \\
\hline 3 & 6 &
\end{array}
$$

Embed $\mathcal{Z}_{\tau} \subset \mathcal{B}_{u}$. Recall the partition $\mathcal{B}_{u}=\bigsqcup_{T} \mathcal{B}_{u}^{T}$ parameterized by standard tableaux, introduced in section 1.1 .

Lemma 3. We have $\mathcal{Z}_{\tau} \subset \mathcal{B}_{u}^{T}$ for $T=\operatorname{st}(\tau)$.

Proof. Let $F=\left(V_{0}, \ldots, V_{n}\right) \in \mathcal{Z}_{\tau}$, and let $\underline{e}=\left(e_{1}, \ldots, e_{n}\right)$ be a $\tau$-basis such that $F=F(\underline{e})$. To prove $F \in \mathcal{B}_{u}^{T}$, fix $i \in\{1, \ldots, \bar{n}\}$ and let us show $Y\left(u_{\mid V_{i}}\right)=Y_{i}^{T}$.

Let $q \geq 1$. The number of boxes in the first $q$ columns of $Y_{i}^{T}$ is equal to the number of entries $j \leq i$ in the first $q$ columns of $T$, which coincide with the entries $j \leq i$ in the first $q$ columns of $\tau$ because $T=\operatorname{st}(\tau)$. The lengths of the rows of the diagram $Y\left(u_{\mid V_{i}}\right)$ are the sizes of the Jordan blocks of $u_{\mid V_{i}}$, it follows that the number of boxes in the first $q$ columns of $Y\left(u_{\mid V_{i}}\right)$ is equal to $\operatorname{dim} \operatorname{ker}\left(u_{\mid V_{i}}\right)^{q}$. Observe that the subspace $\operatorname{ker}\left(u_{\mid V_{i}}\right)^{q}$ is generated by the basic vectors $e_{j}$ associated to the entries $j \leq i$ in the first $q$ columns of $\tau$. Thus both diagrams $Y\left(u_{\mid V_{i}}\right)$ and $Y_{i}^{T}$ 
contain the same number of boxes in their first $q$ columns, for any $q$. Therefore, we have $Y\left(u_{\mid V_{i}}\right)=Y_{i}^{T}$ for any $i$.

As the component $\mathcal{K}^{T} \subset \mathcal{B}_{u}$ is the closure of the subset $\mathcal{B}_{u}^{T}$, we deduce the following

Proposition 4. We have $(\tau, \operatorname{st}(\tau)) \in \mathbf{K}$, for any $\tau$ row-standard.

Next, consider a pair $(\tau, T) \in \mathbf{Y}$. As above, to the row-standard tableau $\tau$, we associate the standard tableau $\operatorname{st}(\tau)$. The tableau $\operatorname{st}(\tau)$ is a fortiori row-standard, and we may consider the pair $(\operatorname{st}(\tau), T) \in \mathbf{Y}$. Then we show the following

Proposition 5. $(\tau, T) \in \mathbf{K} \Rightarrow(\operatorname{st}(\tau), T) \in \mathbf{K}$.

Proof. Write for simplicity $S=\operatorname{st}(\tau)$. Embed $\mathcal{Z}_{\tau}, \mathcal{Z}_{S}, \mathcal{K}^{T}$ in $\mathcal{B}_{u}$ for some $u: V \rightarrow V$ nilpotent. To prove the proposition, it is sufficient to show $\mathcal{Z}_{S} \subset \overline{\mathcal{Z}_{\tau}}$.

Let us consider the elementary operation to arrange in relative order two rows $p<q$ of $\tau$ : let $a_{1}<\ldots<a_{r}$ (resp. $b_{1}<\ldots<b_{s}$ ) be the entries of the $p$-th row (resp. $q$-th row) of the tableau $\tau$. For $i=1, \ldots, s$ write $\tilde{a}_{i}=\min \left(a_{i}, b_{i}\right)$ and $\tilde{b}_{i}=\max \left(a_{i}, b_{i}\right)$. For $i=s+1, \ldots, r$ write $\tilde{a}_{i}=a_{i}$. Then let $\tilde{\tau}$ be the tableau obtained by replacing $a_{i}$ by $\tilde{a}_{i}$ for $i=1, \ldots, r$ and $b_{i}$ by $\tilde{b}_{i}$ for $i=1, \ldots, s$.

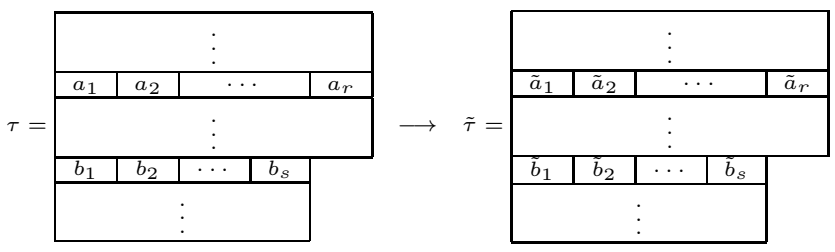

It is clear that this tableau remains row-standard. Observe that $\operatorname{st}(\tilde{\tau})=\operatorname{st}(\tau)=S$. Moreover, the tableau $S$ is obtained from $\tau$ after a sequence of operations as $\tau \mapsto \tilde{\tau}$, hence it is sufficient to show the inclusion $\mathcal{Z}_{\tilde{\tau}} \subset \overline{\mathcal{Z}_{\tau}}$.

Let $F \in \mathcal{Z}_{\tau}$, and let $\underline{e}=\left(e_{1}, \ldots, e_{n}\right)$ be a $\tau$-basis such that $F=F(\underline{e})$. For $i \notin\left\{a_{1}, \ldots, a_{r}, b_{1}, \ldots, b_{s}\right\}$ set $\tilde{e}_{i}=e_{i}$. Set in addition $\tilde{e}_{a_{i}}=e_{\tilde{a}_{i}}$ for $i=1, \ldots, r$, and $\tilde{e}_{b_{i}}=e_{\tilde{b}_{i}}$ for $i=1, \ldots, s$. Then $\underline{\tilde{e}}:=\left(\tilde{e}_{1}, \ldots, \tilde{e}_{n}\right)$ is a $\tilde{\tau}$-basis, and consequently $\tilde{F}:=F(\underline{\tilde{e}})$ belongs to $\mathcal{Z}_{\tilde{\tau}}$. For $t \in \mathbb{C}$ let $d_{t}: V \rightarrow V$ be the automorphism defined by $d_{t}\left(e_{i}\right)=e_{i}$ for $i \notin\left\{b_{1}, \ldots, b_{s}\right\}$, and $d_{t}\left(e_{b_{i}}\right)=e_{b_{i}}+t e_{a_{i}}$. Then $\left(d_{t}\right)_{t \in \mathbb{C}}$ is a one-parameter subgroup of $Z(u)=\{g \in G L(V): g u=u g\}$. Recall that $\mathcal{Z}_{\tau}$ is a $Z(u)$-orbit. Thus the curve $\left\{d_{t} F: t \in \mathbb{C}\right\}$ lies in $\mathcal{Z}_{\tau}$. For all $i=1, \ldots, n$, we have

$d_{t}\left\langle e_{1}, \ldots, e_{i}\right\rangle=\left\langle e_{j}: j \leq i, j \notin\left\{b_{1}, \ldots, b_{s}\right\} ; e_{b_{l}}: a_{l}<b_{l} \leq i ; e_{b_{l}}+t e_{a_{l}}: b_{l} \leq i<a_{l}\right\rangle$,

whence $\lim _{t \rightarrow \infty} d_{t}\left\langle e_{1}, \ldots, e_{i}\right\rangle=\left\langle\tilde{e}_{j}: 1 \leq j \leq i\right\rangle$. It follows $\tilde{F}=\lim _{t \rightarrow \infty} d_{t} F \in \overline{\mathcal{Z}_{\tau}}$. Therefore, $\mathcal{Z}_{\tilde{\tau}}=Z(u) \tilde{F} \subset \overline{\mathcal{Z}_{\tau}}$.

Remark 2. The converse of Proposition 5 is not true. For instance, if

$$
\tau=\begin{array}{|l|l|}
\hline 2 & 3
\end{array} \quad \operatorname{st}(\tau)=\begin{array}{l|l|}
\hline 1 & 3
\end{array} \quad T=\begin{array}{|l|l|}
\hline 1 & 2 \\
\hline 1 &
\end{array} \quad,
$$

then $(\tau, T) \notin \mathbf{K}$, whereas $(\operatorname{st}(\tau), T) \in \mathbf{K}$. This can be seen by applying the criteria which we provide in this paper, or more directly by computing that the component $\mathcal{K}^{T}$ is in this case the set of flags $\left(0 \subset V_{1} \subset V_{2} \subset V_{3}=\mathbb{C}^{3}\right)$ with $V_{1}=\operatorname{Im} u$. 
2.2. Connection to the problem of intersections of components. We connect the problem to determine pairs $(S, T) \in \mathbf{K}$ with $T, S$ both standard to the problem to determine nonempty intersections of components of the Springer fiber. Consider the Springer fiber $\mathcal{B}_{u}$ and an irreducible component $\mathcal{K}^{T} \subset \mathcal{B}_{u}$ associated to the standard tableau $T$. Let $S$ be a standard tableau of same shape as $T$, and let $\mathcal{B}_{u}^{S} \subset \mathcal{B}_{u}$ be the Spaltenstein subset corresponding to $S$ (cf. 1.1). The following lemma will also be used in section 8 .

Lemma 6. $\mathcal{B}_{u}^{S} \cap \mathcal{K}^{T} \neq \emptyset \Leftrightarrow(S, T) \in \mathbf{K}$.

Proof. The implication $(\Leftarrow)$ follows from Lemma 3. The proof of the other implication relies on the following construction.

Let $Y=Y(u)$ be the Young diagram representing the Jordan form of $u$ (see 1.1). As in section 1.2, we consider a Jordan basis $\left(e_{x}\right)_{x \in Y}$ indexed on the boxes of $Y$, with $u\left(e_{x}\right)=0$ for $x$ in the first column of $Y$, and $u\left(e_{x}\right)=e_{x^{\prime}}$, where $x^{\prime}$ is the box on the left of $x$, otherwise. Let $H \subset G L(V)$ be the subgroup of automorphisms which are diagonal in the basis $\left(e_{x}\right)_{x \in Y}$. The flags $F_{\tau}$, parameterized by row-standard tableaux of shape $Y$, introduced in section 1.2, are exactly the elements of the Springer fiber $\mathcal{B}_{u}$ which are fixed by the natural action of $H$ on flags. However, the action of $H$ on flags does not leave $\mathcal{B}_{u}$ stable.

We construct a one-parameter subgroup $H^{\prime} \subset H$ which leaves $\mathcal{B}_{u}$ stable. To do this, we write $x_{p, q}$ the $p$-th box of the $q$-th column of $Y$. Let $e_{p, q}=e_{x_{p, q}}$. Thus

- $u\left(e_{p, q}\right)=0$ for $q=1$,

- $u\left(e_{p, q}\right)=e_{p, q-1}$ otherwise.

Set $\epsilon_{p, q}=n q-p$. Then, we define $h_{t}: V \rightarrow V$ by setting $h_{t}\left(e_{p, q}\right)=t^{\epsilon_{p, q}} e_{p, q}$. Thus $H^{\prime}:=\left(h_{t}\right)_{t \in \mathbb{C}^{*}}$ is a one-parameter subgroup of $H$. One easily checks that $h_{t}\left(u\left(e_{p, q}\right)\right)=t^{-n} u\left(h_{t}\left(e_{p, q}\right)\right)$ for any $t$, hence the action of $H^{\prime}$ leaves $\mathcal{B}_{u}$ and every component of $\mathcal{B}_{u}$ stable. Moreover, as the $\epsilon_{p, q}$ 's are pairwise distinct, the flags $F_{\tau}$ are exactly the fixed points of $\mathcal{B}_{u}$ for the action of $H^{\prime}$.

Let $F \in \mathcal{K}^{T}$. Then for any $t \in \mathbb{C}^{*}$ we have $h_{t} F \in \mathcal{K}^{T}$. It follows $\lim _{t \rightarrow \infty} h_{t} F \in$ $\mathcal{K}^{T}$. Moreover, the $\operatorname{limit} \lim _{t \rightarrow \infty} h_{t} F$ is necessarily a fixed point of $H^{\prime}$, hence $\lim _{t \rightarrow \infty} h_{t} F=F_{\tau}$ for some row-standard tableau $\tau$. To show $(\Rightarrow)$, it is now sufficient to show:

$$
F \in \mathcal{B}_{u}^{S} \Rightarrow F_{\tau}:=\lim _{t \rightarrow \infty} h_{t} F \in \mathcal{B}_{u}^{S} .
$$

Indeed, we then get that, if $\mathcal{B}_{u}^{S} \cap \mathcal{K}^{T}$ is nonempty, it contains an element of the form $F_{\tau}$. So it follows from Lemma 3 that $S=\operatorname{st}(\tau)$, and then from Proposition 5 that $(S, T) \in \mathbf{K}$.

Write $\left\{(p, q): p=1, \ldots, r, q=1, \ldots, \lambda_{p}\right\}=:\left\{\left(p_{i}, q_{i}\right): i=1, \ldots, n\right\}$ so that we have $\epsilon_{p_{1}, q_{1}}<\ldots<\epsilon_{p_{n}, q_{n}}$. Write $e_{i}=e_{p_{i}, q_{i}}$. Let $B \subset G L(V)$ be the Borel subgroup of upper triangular automorphisms. in the basis $\left(e_{1}, \ldots, e_{n}\right)$. Recall that $\mathcal{B}$ denotes the variety of complete flags on $V$. Let $S(\tau) \subset \mathcal{B}$ be the $B$-orbit of $F_{\tau}$ in $\mathcal{B}$. This is a Schubert cell. Then, it is a classical fact that

$$
S(\tau)=\left\{F \in \mathcal{B}: \lim _{t \rightarrow \infty} h_{t} F=F_{\tau}\right\}
$$

Let $P=\left\{g \in G L(V): g\left(\operatorname{ker} u^{q}\right)=\operatorname{ker} u^{q} \forall q\right\}$. This is a parabolic subgroup of $G L(V)$. Notice that $\mathcal{B}_{u}^{S}$ is the intersection between $\mathcal{B}_{u}$ and a $P$-orbit of the flag variety, namely:

$$
\mathcal{P}:=\left\{\left(V_{0}, \ldots, V_{n}\right) \in \mathcal{B}: \operatorname{dim} V_{i} \cap \operatorname{ker} u^{q}=c_{i, q}^{S} \forall i=1, \ldots, n, \forall q\right\},
$$


where $c_{i, q}^{S}$ denotes the number of entries $j \leq i$ in the first $q$ columns of $S$ (cf. the proof of Lemma 3). Observe that $B \subset P$. Thus, $F \in \mathcal{B}_{u}^{S}$ implies

$$
\lim _{t \rightarrow \infty} h_{t} F \in(B F) \cap \mathcal{B}_{u} \subset \mathcal{B}_{u}^{S} .
$$

The proof is now complete.

We deduce the following

Proposition 7. Let $\mathcal{K}^{T}, \mathcal{K}^{T^{\prime}} \subset \mathcal{B}_{u}$ be two components corresponding to standard tableaux $T$ and $T^{\prime}$. We have $\mathcal{K}^{T} \cap \mathcal{K}^{T^{\prime}} \neq \emptyset$ if and only if there is $S$ standard such that $S \in T$ and $S \in T^{\prime}$.

Proof. As $\mathcal{B}_{u}$ is the union of the $\mathcal{B}_{u}^{S}$ 's, the intersection $\mathcal{K}^{T} \cap \mathcal{K}^{T^{\prime}}$ is nonempty if and only if there is some $S$ standard such that $\mathcal{B}_{u}^{S} \cap \mathcal{K}^{T} \cap \mathcal{K}^{T^{\prime}} \neq \emptyset$. By the previous lemma, it is equivalent to have $(S, T),\left(S, T^{\prime}\right) \in \mathbf{K}$.

\section{An inductive PRoperty of the SET K AND STABility by the SCHÜTZENBERGER INVOLUTION}

In this section, we show two combinatorial properties of the set $\mathbf{K}$ of pairs $(\tau, T)$ such that $\tau \in T$. First, in the case where $\tau^{\prime}, T^{\prime}$ are respective subtableaux of $\tau, T$ of same shape, we relate the belonging of $\left(\tau^{\prime}, T^{\prime}\right)$ in $\mathbf{K}$ to the belonging of $(\tau, T)$. Next, we show that the set $\mathbf{K}$ is stable by a combinatorial transformation $(\tau, T) \mapsto\left(S \cdot \tau, T^{S}\right)$ involving the Schützenberger involution $T \mapsto T^{S}$.

3.1. Inductive property. Let $T, T^{\prime}$ be standard tableaux. We write $T^{\prime} \subseteq T$ if $T^{\prime}$ is a subtableau of $T$, i.e. is obtained from $T$ by deleting entries $i, \ldots,|T|$ for some $i \geq 1$. More generally, let $\tau, \tau^{\prime}$ be row-standard tableaux. We write $\tau^{\prime} \subseteq \tau$ if $\left|\tau^{\prime}\right| \leq|\tau|$, and $\tau$ and $\tau^{\prime}$ satisfy the following property: if $i, j$ are entries of $\tau^{\prime}$, then they lie in the same row of $\tau^{\prime}$ if and only if they lie in the same row of $\tau$. For example:

We have the following

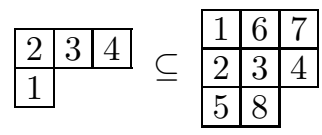

Theorem 8. Let $(\tau, T),\left(\tau^{\prime}, T^{\prime}\right) \in \mathbf{Y}$ and we suppose $\tau^{\prime} \subseteq \tau$ and $T^{\prime} \subseteq T$.

(a) $(\tau, T) \in \mathbf{K} \Rightarrow\left(\tau^{\prime}, T^{\prime}\right) \in \mathbf{K}$.

(b) Moreover, if every $i \in\left\{\left|T^{\prime}\right|+1, \ldots,|T|\right\}$ lies in the same column in $\tau$ and $T$, then $(\tau, T) \in \mathbf{K} \Leftrightarrow\left(\tau^{\prime}, T^{\prime}\right) \in \mathbf{K}$.

Proof of Theorem 8, We first set the notation. Let $n=|T|$ and let $V=\mathbb{C}^{n}$. The component $\mathcal{K}^{T}$ is the closure of the subset $\mathcal{B}_{u}^{T}$ in the Springer fiber $\mathcal{B}_{u}$, for $u \in \operatorname{End}(V)$ nilpotent of appropriate Jordan form. Let $\underline{e}=\left(e_{1}, \ldots, e_{n}\right)$ be a $\tau$ basis and let $F_{\tau}=\left(\left\langle e_{1}, \ldots, e_{i}\right\rangle\right)_{i=0, \ldots, n} \in \mathcal{Z}_{\tau} \subset \mathcal{B}_{u}$ be the corresponding adapted flag. Denote $n^{\prime}=\left|T^{\prime}\right|$ and $V^{\prime}=\left\langle e_{1}, \ldots, e_{n^{\prime}}\right\rangle$. Let $u^{\prime}=u_{\mid V^{\prime}} \in \operatorname{End}\left(V^{\prime}\right)$ be the restriction of $u$ to $V^{\prime}$ and let $\mathcal{B}_{u^{\prime}}$ be the variety of $u^{\prime}$-stable, complete flags of $V^{\prime}$. The component $\mathcal{K}^{T^{\prime}}$ associated to $T^{\prime}$ is the closure of the subset $\mathcal{B}_{u^{\prime}}^{T^{\prime}} \subset \mathcal{B}_{u^{\prime}}$. Moreover, the flag $F_{\tau^{\prime}}:=\left(\left\langle e_{1}, \ldots, e_{i}\right\rangle\right)_{i=0, \ldots, n^{\prime}}$ belongs to the orbit $\mathcal{Z}_{\tau^{\prime}} \subset \mathcal{B}_{u^{\prime}}$.

Let us show part (a) of the theorem: we suppose that $F_{\tau} \in \mathcal{K}^{T}$ and we have to show that $F_{\tau^{\prime}} \in \mathcal{K}^{T^{\prime}}$. We let $Y^{\prime}=\operatorname{sh}\left(T^{\prime}\right)$ and $V^{\prime \prime}=\left\langle e_{n^{\prime}+1}, \ldots, e_{n}\right\rangle$. Let $\mathcal{G}_{n^{\prime}}(V)$ be the variety of $n^{\prime}$-dimensional subspaces of $V$. Let $\mathcal{G}_{Y^{\prime}}(V) \subset \mathcal{G}_{n^{\prime}}(V)$ be the subset 
of subspaces $W$ which are stable by $u$ and such that $Y\left(u_{\mid W}\right)=Y^{\prime}$. This is a locally closed subvariety of $\mathcal{G}_{n^{\prime}}(V)$, which contains $V^{\prime}$, and an open neighborhood of $V^{\prime}$ in $\mathcal{G}_{Y^{\prime}}(V)$ is formed by $\Omega=\left\{W \in \mathcal{G}_{Y^{\prime}}(V): W \cap V^{\prime \prime}=0\right\}$. We rely on the following

Claim. There is an algebraic map $\beta: \Omega \rightarrow G L(V), W \mapsto \beta_{W}$ with the properties:

(1) $\beta_{W}(W)=V^{\prime}$ and $\beta_{W} \circ u(x)=u \circ \beta_{W}(x)$ for all $x \in W$;

(2) $\beta_{V^{\prime}}(x)=x$ for all $x \in V^{\prime}$.

Assume the claim is true. Let $\mathcal{O}=\left\{\left(V_{0}, \ldots, V_{n}\right) \in \mathcal{B}_{u}: V_{n^{\prime}} \in \Omega\right\}$. Then, the map

$$
\Phi: \mathcal{O} \rightarrow \mathcal{B}_{u^{\prime}}, \quad\left(V_{0}, \ldots, V_{n}\right) \mapsto\left(\beta_{V_{n^{\prime}}}\left(V_{0}\right), \ldots, \beta_{V_{n^{\prime}}}\left(V_{n^{\prime}}\right)\right)
$$

is well defined and algebraic. On one hand, we see that $\Phi\left(\mathcal{O} \cap \mathcal{B}_{u}^{T}\right) \subset \mathcal{B}_{u^{\prime}}^{T^{\prime}} \subset \mathcal{K}^{T^{\prime}}$. Note that $\mathcal{O} \cap \mathcal{B}_{u}^{T}$ is open in $\mathcal{B}_{u}^{T}$, hence it is a dense subset of the component $\mathcal{K}^{T}$. Thus, $\Phi\left(\mathcal{O} \cap \mathcal{K}^{T}\right) \subset \mathcal{K}^{T^{\prime}}$. On the other hand, $\Phi\left(F_{\tau}\right)=F_{\tau^{\prime}}$. Therefore, we obtain $F_{\tau^{\prime}} \in \mathcal{K}^{T^{\prime}}$. It remains to show the claim, to complete the proof of part (a).

Proof of the claim. For a subset $S \subset V$, let $\langle S\rangle_{u}=\left\langle u^{l}(x): x \in S, l \geq 0\right\rangle$ be the smallest $u$-stable subspace containing $S$. Thus, the Jordan block decomposition of $V$ can be written $V=\bigoplus_{j=1}^{r}\left\langle e_{i_{j}}\right\rangle_{u}$ where $1 \leq i_{1}, \ldots, i_{r} \leq n$ are pairwise distinct. In turn, the Jordan block decomposition of $V^{\prime}$ can be written $V^{\prime}=\bigoplus_{j=1}^{k}\left\langle e_{i_{j}^{\prime}}\right\rangle_{u}$ with $1 \leq i_{1}^{\prime}, \ldots, i_{k}^{\prime} \leq n^{\prime}$. We may suppose that $\left\langle e_{i_{j}^{\prime}}\right\rangle_{u} \subset\left\langle e_{i_{j}}\right\rangle_{u}$ for all $j$, and, denoting $l_{j}=\operatorname{dim}\left\langle e_{i_{j}^{\prime}}\right\rangle_{u}$, that we have $l_{1} \geq \ldots \geq l_{k}$. Notice that the numbers $l_{1}, \ldots, l_{k}$ are the sizes of the rows of the diagram $Y^{\prime}$.

Suppose that, for each $j \in\{1, \ldots, k\}$, we are given an element $f_{j} \in V^{\prime \prime} \cap \operatorname{ker} u^{l_{j}}$. This implies

$$
f_{j} \in\left\langle e_{i_{j+1}}\right\rangle_{u} \oplus \ldots \oplus\left\langle e_{i_{r}}\right\rangle_{u}
$$

hence the subspaces $\left\langle e_{i_{j}^{\prime}}+f_{j}\right\rangle_{u}$ (for $j=1, \ldots, k$ ) are in direct sum. Also we have $\operatorname{dim}\left\langle e_{i_{j}^{\prime}}+f_{j}\right\rangle_{u}=l_{j}$ for all $j$, thus $W\left(f_{1}, \ldots, f_{k}\right):=\left\langle e_{i_{j}^{\prime}}+f_{j}: j=1, \ldots, k\right\rangle_{u} \in \Omega$.

Now, we fix $W \in \Omega$. For each $j \in\{1, \ldots, k\}$, there is a unique $f_{j} \in V^{\prime \prime}$ (depending algebraically on $W$ ) such that $e_{i_{j}^{\prime}}+f_{j} \in W$. Let us show that

$$
f_{j} \in \operatorname{ker} u^{l_{j}} \text { for all } j \in\{1, \ldots, k\} .
$$

Suppose by contradiction that there is $j$ such that $f_{j} \notin$ ker $u^{l_{j}}$. We take $j$ minimal, so that $f_{1}, \ldots, f_{j-1}$ satisfy (11). Let $j^{\prime} \leq j$ be minimal such that $f_{j} \notin \operatorname{ker} u^{l_{j^{\prime}}}$. The subspaces $\left\langle e_{i_{t}^{\prime}}+f_{t}\right\rangle_{u}$, for $t \in\left\{1, \ldots, j^{\prime}-1, j\right\}$, are in direct sum, and all have dimension bigger than $l_{j^{\prime}}$. Hence $u_{\mid W}$ has $j^{\prime}$ Jordan blocks of length bigger than $l_{j^{\prime}}$. This contradicts the fact that $Y\left(u_{\mid W}\right)=Y^{\prime}$. Therefore, we have shown (2).

From (2), we derive that $W=W\left(f_{1}, \ldots, f_{k}\right)$.

Then, the vectors $u^{l}\left(e_{i_{j}^{\prime}}\right)$ and $u^{l}\left(e_{i_{j}^{\prime}}+f_{j}\right)$, for $j=1, \ldots, k, l=0, \ldots, l_{j}-1$, form bases of $V^{\prime}$ and $W$ respectively. Each one of both is completed into a basis of $V$ by adding the vectors $e_{n^{\prime}+1}, \ldots, e_{n}$. The change of basis isomorphism from the first basis to the second one algebraically depends on $f_{1}, \ldots, f_{k}$, and moreover, using (11), we see that it has determinant 1 . Therefore, also the inverse change of basis isomorphism algebraically depends on $f_{1}, \ldots, f_{k}$, and this is the desired $\beta_{W}$. The claim is proved.

Finally, let us show part (b) of the theorem. It remains to show the implication $(\Leftarrow)$ : we suppose that $F_{\tau^{\prime}} \in \mathcal{K}^{T^{\prime}}$ and we have to show that $F_{\tau} \in \mathcal{K}^{T}$. Let us write 
$V_{i}=\left\langle e_{1}, \ldots, e_{i}\right\rangle$, so that $F_{\tau}=\left(V_{0}, \ldots, V_{n}\right)$ and $F_{\tau^{\prime}}=\left(V_{0}, \ldots, V_{n^{\prime}}\right)$. The map

$$
\Psi: \mathcal{B}_{u^{\prime}} \rightarrow \mathcal{B}_{u}, \quad\left(W_{0}, \ldots, W_{n^{\prime}}\right) \mapsto\left(W_{0}, \ldots, W_{n^{\prime}}, V_{n^{\prime}+1}, \ldots, V_{n}\right),
$$

is clearly well defined and algebraic. It is assumed that every $i \in\left\{n^{\prime}+1, \ldots, n\right\}$ is in the same column in $\tau$ and $T$, this easily implies that the Young diagram $Y\left(u_{\mid V_{i}}\right)$ coincides with $Y_{i}^{T}$ for all $i=n^{\prime}+1, \ldots, n$. It follows that $\Psi\left(\mathcal{B}_{u^{\prime}}^{T^{\prime}}\right) \subset \mathcal{B}_{u}^{T}$. Therefore, $\Psi\left(\mathcal{K}^{T^{\prime}}\right) \subset \mathcal{K}^{T}$, and we derive that $F_{\tau}=\Psi\left(F_{\tau^{\prime}}\right) \in \mathcal{K}^{T}$. The proof of the theorem is now complete.

3.2. Schützenberger involution. We show that the set $\mathbf{K}$ is stabilized by a combinatorial operation $(\tau, T) \mapsto\left(S \cdot \tau, T^{S}\right)$ involving the Schützenberger transform $T^{S}$ of $T$.

Let $T$ be a standard tableau, and let $Y=\operatorname{sh}(T)$ be its shape. For $i=0, \ldots, n$, let $T[i+1, \ldots, n]$ be the skew subtableau of entries $i+1, \ldots, n$. We refer to [4 for the definition of jeu de taquin. Jeu de taquin transforms $T[i+1, \ldots, n]$ into a Young tableau whose shape is a subdiagram $Y_{n / i}^{T} \subset Y$. For example:

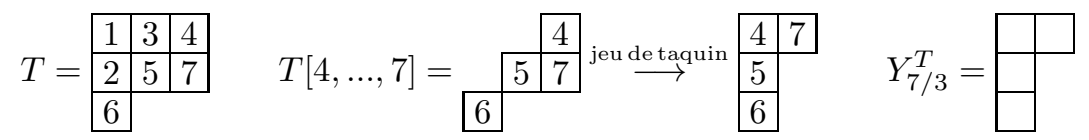

In that way, we obtain an increasing sequence of subdiagrams

$$
\emptyset \subset Y_{n / n-1}^{T} \subset \ldots \subset Y_{n / 0}^{T}=Y .
$$

Let $T^{S}$ be the standard tableau of shape $Y$ obtained by putting $i$ in the new box of $Y_{n / n-i}^{T}$ compared to $Y_{n / n-i+1}^{T}$. The tableau $T^{S}$ is called the Schützenberger transform of $T$. The map $T \mapsto T^{S}$ so-obtained is an involution. For example

$$
T=\begin{array}{|l|l|l}
\hline 1 & 3 & 4 \\
\hline 2 & 5 & 7 \\
\hline 6 & \rightarrow
\end{array} T^{S}=\begin{array}{|l|l|l|}
\hline 1 & 2 & 6 \\
\hline 3 & 5 & 7 \\
\hline 4 &
\end{array}
$$

The Schützenberger transform $T^{S}$ has the following interpretation in terms of components of Springer fibers, which we recall from [8]. For $F=\left(V_{0}, \ldots, V_{n}\right) \in \mathcal{B}_{u}$, let $u_{\mid V_{n} / V_{i}} \in \operatorname{End}\left(V_{n} / V_{i}\right)$ be the nilpotent endomorphism induced by $u$, and let $Y\left(u_{\mid V_{n} / V_{i}}\right)$ be the Young diagram which represents its Jordan form in the sense of section 1.1 Let $Y_{i}^{T}$ be the shape of the subtableau of $T$ with entries $1, \ldots, i$. Define

$$
\mathcal{B}_{u, T}=\left\{F=\left(V_{0}, \ldots, V_{n}\right) \in \mathcal{B}_{u}: Y\left(u_{\mid V_{n} / V_{n-i}}\right)=Y_{i}^{T} \forall i=1, \ldots, n\right\} .
$$

The $\mathcal{B}_{u, T}$ 's form a partition of the variety $\mathcal{B}_{u}$. By [8, Theorem 3.3], the closure of $\mathcal{B}_{u, T}$ is the irreducible component $\mathcal{K}^{T^{S}} \subset \mathcal{B}_{u}$ associated to the tableau $T^{S}$.

Now, let $\tau$ be a row-standard tableau. Let $S \cdot \tau$ denote the row-standard tableau constructed as follows. First, replace each entry $i$ in $\tau$ by $n-i+1$. Then, reverse the order of the entries inside each row, so that the tableau so-obtained becomes row-standard. The map $\tau \mapsto S \cdot \tau$ is clearly an involution. For example:

$$
\tau=\begin{array}{|l|l|l|}
\hline 3 & 4 & 7 \\
\hline 1 & 5 & 6 \\
\hline 2 & \longrightarrow
\end{array} \rightarrow \tau \cdot \tau=\begin{array}{|l|l|l|}
\hline 1 & 4 & 5 \\
\hline 2 & 3 & 7 \\
\hline 6 & \multicolumn{2}{|c}{} \\
\hline
\end{array}
$$

We get finally an involutive map $\mathbf{Y} \rightarrow \mathbf{Y},(\tau, T) \mapsto\left(S \cdot \tau, T^{S}\right)$. We show:

Proposition 9. We have $(\tau, T) \in \mathbf{K}$ if and only if $\left(S \cdot \tau, T^{S}\right) \in \mathbf{K}$. 
Proof. Embed the component $\mathcal{K}^{T}$ and the orbit $\mathcal{Z}_{\tau}$ in the Springer fiber $\mathcal{B}_{u}$ corresponding to some nilpotent $u \in \operatorname{End}(V)$. Let $V^{*}$ be the dual vector space of $V$ and let $u^{*} \in \operatorname{End}\left(V^{*}\right)$ be the dual (nilpotent) endomorphism. Let $\mathcal{B}_{u^{*}}$ be the corresponding Springer fiber. Both $u$ and $u^{*}$ have the same Jordan shape, hence $\mathcal{B}_{u}$ and $\mathcal{B}_{u^{*}}$ are isomorphic. Let $\mathcal{K}^{* T^{S}} \subset \mathcal{B}_{u^{*}}$ be the component of $\mathcal{B}_{u^{*}}$ associated to $T^{S}$, and let $\mathcal{Z}_{S \cdot \tau}^{*} \subset \mathcal{B}_{u^{*}}$ be the $Z\left(u^{*}\right)$-orbit of $\mathcal{B}_{u^{*}}$ associated to $S \cdot \tau$. For a subspace $W \subset V$, write $W^{\perp}=\left\{\phi \in V^{*}: \phi(w)=0 \forall w \in W\right\}$. Let $\Phi: \mathcal{B}_{u} \rightarrow \mathcal{B}_{u^{*}}$ be the map which sends $\left(V_{0}, \ldots, V_{n}\right)$ to $\left(V_{n}^{\perp}, \ldots, V_{0}^{\perp}\right)$. It is an involutive isomorphism. On one hand, notice that whenever $W \subset V$ is a $u$-stable subspace, the restriction $u_{\mid W} \in \operatorname{End}(W)$ and the map $\left(u^{*}\right)_{\mid V^{*} / W^{\perp}} \in \operatorname{End}\left(V^{*} / W^{\perp}\right)$ induced by $u^{*}$ have the same Jordan form. This implies that $\Phi\left(\mathcal{B}_{u}^{T}\right)=\mathcal{B}_{u^{*}, T}$, whence the equality $\Phi\left(\mathcal{K}^{T}\right)=\mathcal{K}^{* T^{S}}$. On the other hand, let $\underline{e}=\left(e_{1}, \ldots, e_{n}\right)$ be a $\tau$-basis of $V$ and let $\underline{e^{*}}=\left(e_{1}^{*}, \ldots, e_{n}^{*}\right)$ be its dual basis. Then $\underline{e^{* \prime}}=\left(e_{n}^{*}, \ldots, e_{1}^{*}\right)$ is a $(S \cdot \tau)$-basis of $V^{*}$ and we see that $\Phi(F(\underline{e}))=F\left(\underline{e^{* \prime}}\right)$. It follows $\Phi\left(\mathcal{Z}_{\tau}\right)=\mathcal{Z}_{S \cdot \tau}^{*}$. We get finally $\mathcal{Z}_{\tau} \subset \mathcal{K}^{T}$ $\Leftrightarrow \mathcal{Z}_{S \cdot \tau}^{*} \subset \mathcal{K}^{* T^{S}}$. Therefore, $(\tau, T) \in \mathbf{K} \Leftrightarrow\left(S \cdot \tau, T^{S}\right) \in \mathbf{K}$.

\section{The general inclusion $\mathbf{K} \subset \mathbf{R}$}

In this section, we associate to a row-standard tableau $\tau$ a sequence of Young diagrams $Y_{j / i}(\tau)$ for $0 \leq i<j \leq n$, and we associate to a standard tableau $T$ a sequence of Young diagrams $Y_{j / i}^{T}$ for $0 \leq i<j \leq n$ (cf. 4.2). We write

$$
\tau \preceq T \text { if } Y_{j / i}(\tau) \preceq Y_{j / i}^{T} \text { for any } i, j,
$$

where $\preceq$ is the dominance relation. The set

$$
\mathbf{R}:=\{(\tau, T) \in \mathbf{Y}: \tau \preceq T\}
$$

has a purely combinatorial definition.

Actually, the diagrams $Y_{j / i}(\tau)$ (resp. $Y_{j / i}^{T}$ ) represent the Jordan forms of the nilpotent maps induced by $u$ on the subquotients $V_{j} / V_{i}$ of a flag $F \in \mathcal{Z}_{\tau}$ (resp. of a generic element $F \in \mathcal{K}^{T}$ ).

We show the general implication

$$
\tau \in T \Rightarrow \tau \preceq T,
$$

which means that we have the inclusion $\mathbf{K} \subset \mathbf{R}$ (cf. Theorem 11). This provides a combinatorial necessary condition for having $\tau \in T$. However, this condition is not sufficient whenever $\tau, T$ are not of hook, two-row or two-column type (cf. Proposition 12).

4.1. Lower semi-continuity of the Jordan form on a subquotient. The set of Young diagrams is partially ordered with the dominance order: let $Y, Y^{\prime}$ be two Young diagrams, let $\lambda_{1} \geq \ldots \geq \lambda_{r}$ (resp. $\lambda_{1}^{\prime} \geq \ldots \geq \lambda_{r}^{\prime}$ ) be the lengths - possibly 0 - of the rows of $Y$ (resp. of $Y^{\prime}$ ), we write $Y \preceq Y^{\prime}$ if

$$
\lambda_{1}+\ldots+\lambda_{p} \leq \lambda_{1}^{\prime}+\ldots+\lambda_{p}^{\prime} \forall p=1, \ldots, r .
$$

Equivalently, denoting by $\mu_{1} \geq \ldots \geq \mu_{s}$ (resp. $\mu_{1}^{\prime} \geq \ldots \geq \mu_{s}^{\prime}$ ) the lengths of the columns of $Y$ (resp. $Y^{\prime}$ ), we have $Y \preceq Y^{\prime}$ if and only if

$$
\mu_{1}^{\prime}+\ldots+\mu_{q}^{\prime} \leq \mu_{1}+\ldots+\mu_{q} \forall q=1, \ldots, s .
$$


Recall from 1.1 that, for $F=\left(V_{0}, \ldots, V_{n}\right) \in \mathcal{B}_{u}$, we denote by $Y\left(u_{\mid V_{i}}\right)$ the diagram representing the Jordan form of the nilpotent $u_{\mid V_{i}} \in \operatorname{End}\left(V_{i}\right)$ induced by $u$. Observe that the map $F \mapsto Y\left(u_{\mid V_{i}}\right)$ is lower semicontinuous, i.e. the subset

$$
\left\{F \in \mathcal{B}_{u}: Y\left(u_{\mid V_{i}}\right) \preceq Y_{0}\right\}
$$

is closed for any Young diagram $Y_{0}$. Indeed, we see that the number of boxes in the first $q$ columns of $Y\left(u_{\mid V_{i}}\right)$ is $\operatorname{dim} \operatorname{ker}\left(u_{\mid V_{i}}\right)^{q}=n-\operatorname{rk}\left(u_{\mid V_{i}}\right)^{q}$, and the map $F \mapsto \operatorname{rk}\left(u_{\mid V_{i}}\right)^{q}$ is lower semicontinuous. Therefore, we get an implication:

$$
F \in \mathcal{K}^{T} \Rightarrow Y\left(u_{\mid V_{i}}\right) \preceq Y_{i}^{T} \forall i=1, \ldots, n .
$$

More generally, for $0 \leq i<j \leq n$ and for $F=\left(V_{0}, \ldots, V_{n}\right) \in \mathcal{B}_{u}$, we may consider the nilpotent map $u_{\mid V_{j} / V_{i}} \in \operatorname{End}\left(V_{j} / V_{i}\right)$ induced by $u$ on the subquotient $V_{j} / V_{i}$, and its Young diagram $Y\left(u_{\mid V_{j} / V_{i}}\right)$.

Lemma 10. The map $F=\left(V_{0}, \ldots, V_{n}\right) \mapsto Y\left(u_{\mid V_{j} / V_{i}}\right)$ is lower semicontinuous.

Proof. It is sufficient to show that the map $F \mapsto \operatorname{rk} u_{\mid V_{j} / V_{i}}^{q}$ is lower semicontinuous. This is an easy consequence of the formula

$$
\operatorname{rk} u_{\mid V_{j} / V_{i}}^{q}=\operatorname{dim}\left(V_{i}+u^{q}\left(V_{j}\right)\right)-i
$$

which can be proved by using the rank formula (see [2, Lemma 2.2]).

4.2. A combinatorial necessary condition for having $\tau \in T$. In the purpose to deduce from Lemma 10 a necessary condition for having $\tau \in T$, we determine $Y\left(u_{\mid V_{j} / V_{i}}\right)$ for $F \in \mathcal{Z}_{\tau}$ and for a generic $F \in \mathcal{K}^{T}$.

Let $\tau$ be a row-standard tableau, with $|\tau|=n$ boxes. Let $0 \leq i<j \leq n$. Let $m_{1} \geq \ldots \geq m_{k}$ be the sizes of the rows of the subtableau $\tau[i+1, \ldots, j]$ of entries $i+1, \ldots, j$. Define $Y_{j / i}(\tau)$ as the Young diagram of rows of lengths $m_{1}, \ldots, m_{k}$. For example

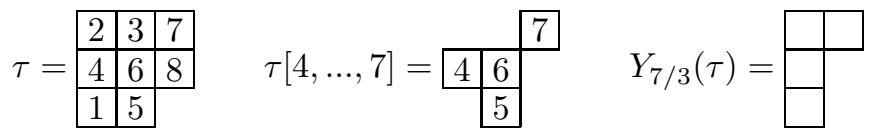

Embed $\mathcal{Z}_{\tau} \subset \mathcal{B}_{u}$. Let $F=\left(V_{0}, \ldots, V_{n}\right) \in \mathcal{Z}_{\tau}$ and let $\underline{e}=\left(e_{1}, \ldots, e_{n}\right)$ be a $\tau$-basis such that $F=F(\underline{e})$. Then we see that the subbasis $\left(e_{i+1}, \ldots, e_{j}\right)$ forms a Jordan basis of $u_{\mid V_{j} / V_{i}}$ of Jordan blocks of sizes $m_{1}, \ldots, m_{k}$. We have thus

$$
Y\left(u_{\mid V_{j} / V_{i}}\right)=Y_{j / i}(\tau) \text {. }
$$

Let $T$ be a standard tableau, with $|T|=n$ boxes. Let $0 \leq i<j \leq n$. The subtableau $T[i+1, \ldots, j]$ of entries $i+1, \ldots, j$ is a skew tableau. By jeu de taquin, we transform it into a Young tableau. We denote by $Y_{j / i}^{T}$ the shape of the soobtained Young tableau. For example

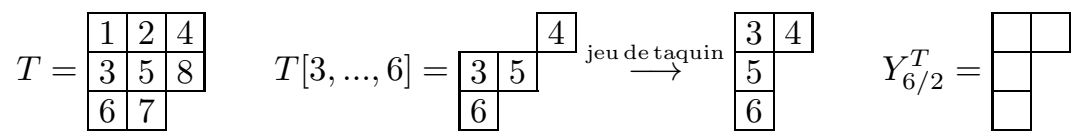

Embed $\mathcal{K}^{T} \subset \mathcal{B}_{u}$. It follows from [8, Theorem 3.3] that the set

$$
\left\{\left(V_{0}, \ldots, V_{n}\right) \in \mathcal{K}^{T}: Y\left(u_{\mid V_{j} / V_{i}}\right)=Y_{j / i}^{T}\right\}
$$

is a nonempty open subset of the component $\mathcal{K}^{T}$.

Let $(\tau, T) \in \mathbf{Y}$. We write

$$
\tau \preceq T \text { if } Y_{j / i}(\tau) \preceq Y_{j . i}^{T} \text { for all } 0 \leq i<j \leq n .
$$


Define $\mathbf{R}$ as the set of pairs $(\tau, T) \in \mathbf{Y}$ such that $\tau \preceq T$. By Lemma 10 we get:

Theorem 11. We have $\mathbf{K} \subset \mathbf{R}$.

4.3. The condition is not sufficient. The equivalence $\tau \in T \Leftrightarrow \tau \preceq T$ does not hold in general. As a first example, let

$$
\tau=\begin{array}{l|l|l|l|l|l|l|}
\hline 1 & 2 & 5 \\
\hline 4 & 6 &
\end{array} \quad T=\begin{array}{llll}
\hline 1 & 2 & 5 \\
\hline 3 & & 4 & \\
\hline 6 & &
\end{array}
$$

Then, we have $\tau \preceq T$ but $\tau \notin T$. Indeed, embed $\mathcal{K}^{T}, \mathcal{Z}_{\tau} \subset \mathcal{B}_{u}$. First, note that $\operatorname{dim} \operatorname{ker} u \cap \operatorname{Im} u=2$. On one hand, we have $\operatorname{ker} u \cap \operatorname{Im} u \subset V_{3}$ for all $\left(V_{0}, \ldots, V_{6}\right) \in$ $\mathcal{B}_{u}^{T}$, hence also for all $\left(V_{0}, \ldots, V_{6}\right) \in \mathcal{K}^{T}$. On the other hand, $\operatorname{dim}\left(V_{3} \cap \operatorname{ker} u \cap \operatorname{Im} u\right)=$ 1 for any $\left(V_{0}, \ldots, V_{6}\right) \in \mathcal{Z}_{\tau}$. It follows: $\mathcal{Z}_{\tau} \not \subset \mathcal{K}^{T}$.

Actually, we have the following

Proposition 12. Let $Y$ be a Young diagram containing $\square$ as a subdiagram. Then there is $(\tau, T)$ with $Y=\operatorname{sh}(\tau)=\operatorname{sh}(T)$, such that $\tau \preceq T$ and $\tau \notin T$.

Proof. First, let $Y$ be a Young diagram of rows of lengths $\left(\lambda_{1}, \lambda_{2}, \ldots, \lambda_{r}\right)$ with $\lambda_{1}>\lambda_{2} \geq 2, \lambda_{3} \geq 1, r \geq 3$. Let $R_{1}$ be a row of $\lambda_{1}-3$ empty boxes, let $R_{2}$ be a row of $\lambda_{2}-2$ boxes, let $R_{3}$ be a row of $\lambda_{3}-1$ boxes, for $p \geq r$ let $R_{p}$ be a row of $\lambda_{p}$ boxes. Fill in the boxes of $R_{1}$ with numbers $7,8, \ldots, \lambda_{1}+3$ from left to right, number $R_{2}$ in turn by $\lambda_{1}+4, \lambda_{1}+5, \ldots, 1+\lambda_{1}+\lambda_{2}$ from left to right, number $R_{3}$ by $\lambda_{1}+\lambda_{2}+2, \ldots, \lambda_{1}+\lambda_{2}+\lambda_{3}$ from left to right, for $p \geq r$ number $R_{p}$ by $\lambda_{1}+\ldots+\lambda_{p-1}+1, \ldots, \lambda_{1}+\ldots+\lambda_{p}$ from left to right. It is straightforward to check that the pair $(\tau, T)$ with
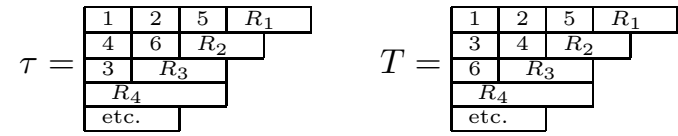

belongs to $\mathbf{R}$. By Theorem 8 , it does not belong to $\mathbf{K}$.

It is straightforward to deduce that the pair $(\hat{\tau}, \hat{T})$ with

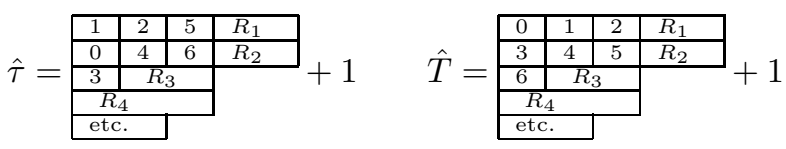

belongs to $\mathbf{R}$ (where " +1 " means that we increase by 1 each entry of the tableau, in order to obtain a row-standard and a standard tableau). (The dominance relations for $(i, j)$ for $i \geq 1$ follow from the previous case - relations for $(0, j)$ are easy.) Let us show $(\hat{\tau}, \hat{T}) \notin \mathbf{K}$. Let $\left(\hat{\tau}^{\prime}, \hat{T}^{\prime}\right)$ be the pair of subtableaux of entries $1, \ldots, 7$ :

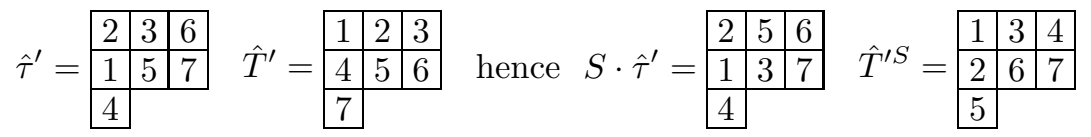

Let $\hat{\tau}^{\prime \prime}=S \cdot\left(\left(S \cdot \hat{\tau}^{\prime}\right)[1, \ldots, 6]\right)$ and $\hat{T}^{\prime \prime}=\left(\left(\hat{T}^{\prime S}\right)[1, \ldots, 6]\right)^{S}$. We have

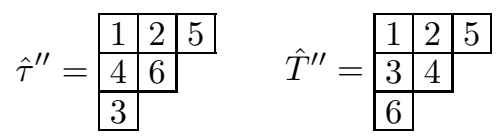


LUCAS FRESSE

As observed previously, we have $\left(\hat{\tau}^{\prime \prime}, \hat{T}^{\prime \prime}\right) \notin \mathbf{K}$. Combining Theorem 8 and Proposition 9, we deduce $(\tau, T) \notin \mathbf{K}$. It solves the case $\left(\lambda_{1}, \lambda_{2}, \ldots, \lambda_{r}\right)$ with $\lambda_{1}=\lambda_{2} \geq 3$, $\lambda_{3} \geq 1, r \geq 3$. The proof is now complete.

\section{Equivalence $\tau \in T \Leftrightarrow \tau \preceq T$ IN THE HOOK, 2-ROW AND 2-COLUmN CASES}

In the previous section we have established the general implication

$$
\tau \in T \Rightarrow \tau \preceq T .
$$

Proposition 12 shows that the inverse implication is not true in general. Our purpose in this section is to show the following

Theorem 13. Let $(\tau, T) \in \mathbf{Y}$ be of hook, two-row or two-column type. We have the equivalence

$$
\tau \in T \Leftrightarrow \tau \preceq T .
$$

We treat successively the three cases, and Theorem 13 will follow by combining Theorems 14, 15] and 16. The proof in each case relies on a further characterization.

5.1. Equivalence in the hook case. Let us state some notation. We denote by $\mathbf{Y}_{\text {hk }}$ the subset of pairs $(\tau, T) \in \mathbf{Y}$ such that the Young shape $\operatorname{sh}(\tau)=\operatorname{sh}(T)$ is of hook type. Set $\mathbf{K}_{\mathrm{hk}}=\mathbf{K} \cap \mathbf{Y}_{\mathrm{hk}}$ and $\mathbf{R}_{\mathrm{hk}}=\mathbf{R} \cap \mathbf{Y}_{\mathrm{hk}}$. We aim to prove the equality $\mathbf{K}_{\mathrm{hk}}=\mathbf{R}_{\mathrm{hk}}$.

We rely on another subset $\mathbf{A}_{\mathrm{hk}} \subset \mathbf{Y}_{\mathrm{hk}}$. Let $(\tau, T) \in \mathbf{Y}_{\mathrm{hk}}$. Let $a_{1}<\ldots<a_{s}$ (resp. $a_{1}^{\prime}<\ldots<a_{s}^{\prime}$ ) be the entries in the first row of $T$ (resp. of $\tau$ ). Say $(\tau, T) \in \mathbf{A}_{\mathrm{hk}}$ if we have

$$
a_{q-1}^{\prime}<a_{q} \leq a_{q}^{\prime} \forall q \in\{2, \ldots, s\} .
$$

We show the following

Theorem 14. We have $\mathbf{K}_{\mathrm{hk}}=\mathbf{A}_{\mathrm{hk}}=\mathbf{R}_{\mathrm{hk}}$.

Proof. The inclusion $\mathbf{K}_{\mathrm{hk}} \subset \mathbf{R}_{\mathrm{hk}}$ follows from Theorem 11. The inclusion $\mathbf{A}_{\mathrm{hk}} \subset$ $\mathbf{K}_{\mathrm{hk}}$ follows from [18, Theorem 4.1]. Then it remains to show $\mathbf{R}_{\mathrm{hk}} \subset \mathbf{A}_{\mathrm{hk}}$. Suppose that $(\tau, T) \in \mathbf{R}_{\mathrm{hk}}$ and let us show $(\tau, T) \in \mathbf{A}_{\mathrm{hk}}$. Let $q \in\{2, \ldots, s\}$. Define $\hat{q} \in$ $\{1, \ldots, s+1\}$ by

- if $a_{s}^{\prime} \geq a_{q}$, then $\hat{q} \in\{1, \ldots, s\}$ is minimal such that $a_{\hat{q}}^{\prime} \geq a_{q}$,

- if $a_{s}^{\prime}<a_{q}$, then $\hat{q}=s+1$.

We have to prove $q=\hat{q}$.

First, we use the relation $Y_{a_{q}-1 / 0}(\tau) \preceq Y_{a_{q}-1 / 0}^{T}$. The diagram $Y_{a_{q}-1 / 0}^{T}$ is the shape of the subtableau of $T$ of entries $i<a_{q}$, hence its first row contains $q-1$ boxes. The lengths of the rows of the diagram $Y_{a_{q}-1 / 0}(\tau)$ are the lengths of the rows of the subtableau of $\tau$ of entries $i<a_{q}$. The first row of this subtableau contains $\hat{q}-1$ boxes. By the relation above, we get $\hat{q} \leq q$.

Write $n=|\tau|=|T|$. Next, we use the relation $Y_{n / a_{q}-1}(\tau) \preceq Y_{n / a_{q}-1}^{T}$. The diagram $Y_{n / a_{q}-1}^{T}$ is the shape of the transform by jeu de taquin of the skew subtableau of $T$ of entries $a_{q}, \ldots, n$, that is

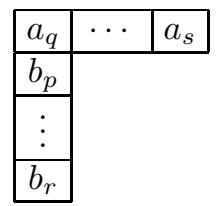


for some $b_{p}, \ldots, b_{r}$. Thus the first row of $Y_{n / a_{q}-1}^{T}$ has length $s-q+1$. On the other hand the first row of the subtableau of $\tau$ of entries $a_{q}, \ldots, n$ contains the entries $a_{\hat{q}}^{\prime}, \ldots, a_{s}^{\prime}$. This row is nonempty, since we have already proved $\hat{q} \leq q(\leq s)$. The first row of the Young diagram $Y_{n / a_{q}-1}(\tau)$ has thus length $s-\hat{q}+1$. By the dominance relation above, we obtain $\hat{q} \geq q$. We get finally $\hat{q}=q$.

5.2. Equivalence in the two-row case. We denote by $\mathbf{Y}_{2 \text {-r }}$ the subset of pairs $(\tau, T) \in \mathbf{Y}$ such that the Young $\operatorname{shape} \operatorname{sh}(\tau)=\operatorname{sh}(T)$ has (at most) two rows. Set $\mathbf{K}_{2-\mathrm{r}}=\mathbf{K} \cap \mathbf{Y}_{2-\mathrm{r}}$ and $\mathbf{R}_{2-\mathrm{r}}=\mathbf{R} \cap \mathbf{Y}_{2-\mathrm{r}}$. We shall prove the equality $\mathbf{K}_{2-\mathrm{r}}=\mathbf{R}_{2-\mathrm{r}}$. As previously for the hook case, we rely on another subset $\mathbf{A}_{2 \text {-r }} \subset \mathbf{Y}_{2 \text {-r }}$ that we define by induction.

First, we define $\hat{\mathbf{A}}_{2-\mathrm{r}}$ as the set of $(\tau, T) \in \mathbf{Y}_{\text {2-r }}$ such that one of the following conditions is satisfied (writing $n=|\tau|=|T|$ ):

(1) there is $i \in\{2, \ldots, n\}$ (necessarily even) such that the subtableaux $\tau[1, \ldots, i]$ and $T[1, \ldots, i]$ of entries $1, \ldots, i$ have a rectangular shape with two rows of length $i / 2$;

(2) the subtableau $T[1, \ldots, i]$ has a non-rectangular shape for every $i \in\{2, \ldots, n\}$ and 1 has the same place in $\tau$ and $T$.

Let $(\tau, T) \in \hat{\mathbf{A}}_{2-\mathrm{r}}$. We associate to the pair $(\tau, T)$ a subset $\eta(\tau, T) \subset \mathbf{Y}_{2-\mathrm{r}}$ with one or two elements:

(a) Suppose that (1) holds for some $i<n$, and choose $i \in\{2, \ldots, n-1\}$ maximal for which the property holds. Then the subtableaux $\tau^{\prime}:=\tau[1, \ldots, i]$ and $T^{\prime}:=$ $T[1, \ldots, i]$ have the same shape. On the other hand, the subtableaux $\tau[i+1, \ldots, n]$ and $T[i+1, \ldots, n]$ of entries $i+1, \ldots, n$ have the same shape which is a Young diagram with $n-i$ boxes. Write $\tau^{\prime \prime}=\tau[i+1, \ldots, n]-i$ and $T^{\prime \prime}=T[i+1, \ldots, n]-i$, where " $-i$ " means that each entry $j$ in the tableau is replaced by $j-i$, so that $\tau^{\prime \prime}$ and $T^{\prime \prime}$ are respectively row-standard and standard. Define $\eta(\tau, T)=\left\{\left(\tau^{\prime}, T^{\prime}\right),\left(\tau^{\prime \prime}, T^{\prime \prime}\right)\right\}$.

(b) Suppose now that (1) holds for no $i<n$, but holds for $i=n$. Write $T^{\prime}=$ $T[1, \ldots, n-1]$. Let $\tilde{\tau}$ be the tableau obtained by switching the two rows of $\tau$. The entry $n$ is either in the second row of $\tau$, or in the second row of $\tilde{\tau}$. In the former case, write $\tau^{\prime}=\tau[1, \ldots, n-1]$. In the latter case, write $\tau^{\prime}=\tilde{\tau}[1, \ldots, n-1]$. Define $\eta(\tau, T)=\left\{\left(\tau^{\prime}, T^{\prime}\right)\right\}$.

(c) Finally, suppose that (2) holds. Write $1=a_{1}<a_{2}<\ldots<a_{r}$ (resp. $b_{1}<$ $b_{2}<\ldots<b_{s}$ ) the entries of the first (resp. second) row of $T$. By hypothesis, we have $s<r$ and $b_{q}>a_{q+1}$ for any $q=1, \ldots, s$, hence the transform by jeu de taquin of the subtableau $T[2, \ldots, n]$ contains $a_{2}, \ldots, a_{r}$ in its first row and $b_{1}, \ldots, b_{s}$ in its second row. Let $T^{\prime}$ be the standard tableau with $a_{2}-1, \ldots, a_{r}-1$ in its first row and $b_{1}-1, \ldots, b_{s}-1$ in its second row. Write $1=a_{1}^{\prime}<a_{2}^{\prime}<\ldots<a_{r}^{\prime}$ (resp. $b_{1}^{\prime}<b_{2}^{\prime}<\ldots<b_{s}^{\prime}$ ) the entries of the first (resp. second) row of $\tau$. Let $\tau^{\prime}$ be the row-standard tableau with $a_{2}^{\prime}-1, \ldots, a_{r}^{\prime}-1$ in its first row and $b_{1}^{\prime}-1, \ldots, b_{s}^{\prime}-1$ in its second row. Define $\eta(\tau, T)=\left\{\left(\tau^{\prime}, T^{\prime}\right)\right\}$.

Now, we define the set $\mathbf{A}_{2-r}$ by induction.

- Say $(1,1,1) \in \mathbf{A}_{2-\mathrm{r}}$.

- For $(\tau, T) \in \mathbf{Y}$ with $|\tau|=|T|>1$, say $(\tau, T) \in \mathbf{A}_{2 \text {-r }}$ if we have $(\tau, T) \in \hat{\mathbf{A}}_{2 \text {-r }}$ and $\eta(\tau, T) \subset \mathbf{A}_{2-\mathrm{r}}$. 
Example 1. Let

$$
\tau=\begin{array}{l|l|l}
\hline 2 & 3 & 5
\end{array} \quad T=\begin{array}{|l|l|l|}
\hline 1 & 3 & 4 \\
\hline 1 & 4 & 5
\end{array}
$$

We see that $(\tau, T) \in \hat{\mathbf{A}}_{2-\mathrm{r}}$, and $\eta(\tau, T)=\left\{\left(\tau^{\prime}, T^{\prime}\right),\left(\tau^{\prime \prime}, T^{\prime \prime}\right)\right\}$ with

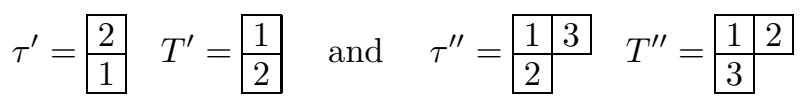

We have $\left(\tau^{\prime}, T^{\prime}\right),\left(\tau^{\prime \prime}, T^{\prime \prime}\right) \in \hat{\mathbf{A}}_{2 \text {-r }}$ and

$$
\begin{aligned}
& \eta\left(\tau^{\prime}, T^{\prime}\right)=(1,1) \in \mathbf{A}_{2-\mathrm{r}}, \text { hence }\left(\tau^{\prime}, T^{\prime}\right) \in \mathbf{A}_{2-\mathrm{r}}, \\
& \eta\left(\tau^{\prime \prime}, T^{\prime \prime}\right)=\left(\frac{2}{1}, \frac{1}{2}\right)=\left(\tau^{\prime}, T^{\prime}\right) \in \mathbf{A}_{2-\mathrm{r}}, \text { hence }\left(\tau^{\prime \prime}, T^{\prime \prime}\right) \in \mathbf{A}_{2-\mathrm{r}} .
\end{aligned}
$$

Thus we get $\eta(\tau, T) \subset \mathbf{A}_{2-\mathrm{r}}$, and it finally results $(\tau, T) \in \mathbf{A}_{2-\mathrm{r}}$.

We prove the following

Theorem 15. We have $\mathbf{K}_{2-\mathrm{r}}=\mathbf{A}_{2-\mathrm{r}}=\mathbf{R}_{2-\mathrm{r}}$.

Proof. I) The inclusion $\mathbf{K}_{2-\mathrm{r}} \subset \mathbf{R}_{2-\mathrm{r}}$ follows from Theorem 11,

II) Let us show the inclusion $\mathbf{R}_{2-\mathrm{r}} \subset \mathbf{A}_{2-\mathrm{r}}$. According to the inductive definition of $\mathbf{A}_{2-r}$, it is sufficient to check that any $(\tau, T) \in \mathbf{R}_{2-\mathrm{r}}$ satisfies $(\tau, T) \in \hat{\mathbf{A}}_{2-\mathrm{r}}$ and $\eta(\tau, T) \subset \mathbf{R}_{2-\mathrm{r}}$.

Set $n=|\tau|=|T|$. If for some $i \in\{2, \ldots, n\}$, the subtableau $T[1, \ldots, i]$ is rectangular, then, using the relation $Y_{i / 0}(\tau) \preceq Y_{i / 0}^{T}$, we get that the subtableau $\tau[1, \ldots, i]$ is also rectangular. If $T[1, \ldots, i]$ is rectangular for none $i \in\{2, \ldots, n\}$, then, using the relation $Y_{n / 1}(\tau) \preceq Y_{n / 1}^{T}$, we get that 1 is in the first row of $\tau$. Thus $(\tau, T) \in \hat{\mathbf{A}}_{2-\mathrm{r}}$. In case (a) of the definition of $\eta(\tau, T)$, we have $Y_{l / k}\left(\tau^{\prime}\right)=Y_{l / k}(\tau)$ for any $0 \leq k<$ $l \leq i$ and $Y_{l / k}\left(\tau^{\prime \prime}\right)=Y_{l+i / k+i}(\tau)$ for any $0 \leq k<l \leq n-i$ on one hand, $Y_{l / k}^{T^{\prime}}=Y_{l / k}^{T}$ for any $0 \leq k<l \leq i$ and $Y_{l / k}^{T^{\prime \prime}}=Y_{l+i / k+i}^{T}$ for any $0 \leq k<l \leq n-i$ on the other hand, therefore $\left(\tau^{\prime}, T^{\prime}\right),\left(\tau^{\prime \prime}, T^{\prime \prime}\right) \in \mathbf{R}_{2-\mathrm{r}}$.

In case (b) of the definition of $\eta(\tau, T)$, we have $Y_{l / k}\left(\tau^{\prime}\right)=Y_{l / k}(\tau)$ for any $0 \leq k<$ $l \leq n-1$ and $Y_{l / k}^{T^{\prime}}=Y_{l / k}^{T}$ for any $0 \leq k<l \leq n-1$, hence $\left(\tau^{\prime}, T^{\prime}\right) \in \mathbf{R}_{2-\mathrm{r}}$.

In case (c) of the definition of $\eta(\tau, T)$, we have $Y_{l / k}\left(\tau^{\prime}\right)=Y_{l+1 / k+1}(\tau)$ for any $0 \leq k<l \leq n-1$ and $Y_{l / k}^{T^{\prime}}=Y_{l+1 / k+1}^{T}$ for any $0 \leq k<l \leq n-1$, hence $\left(\tau^{\prime}, T^{\prime}\right) \in \mathbf{R}_{2-\mathrm{r}}$.

In all the cases, we have shown $\eta(\tau, T) \subset \mathbf{R}_{2-\mathrm{r}}$. It follows $\mathbf{R}_{2-\mathrm{r}} \subset \mathbf{A}_{2-\mathrm{r}}$.

III) Now, let us prove the inclusion $\mathbf{A}_{2-\mathrm{r}} \subset \mathbf{K}_{2-\mathrm{r}}$. To do this, it is sufficient to show that $\eta(\tau, T) \subset \mathbf{K}_{2-\mathrm{r}}$ implies $(\tau, T) \in \mathbf{K}_{2-\mathrm{r}}$, for any $(\tau, T) \in \hat{\mathbf{A}}_{2-\mathrm{r}}$.

In case (c) of the definition of $\eta(\tau, T)$, we have $\tau^{\prime}=S \cdot((S \cdot \tau)[1, \ldots, n-1])$ and $T^{\prime}=\left(\left(T^{S}\right)[1, \ldots, n-1]\right)^{S}$, and $n$ is in the same place of $S \cdot \tau$ and $T^{S}$, hence, combining Theorem 8(b) and Proposition 9, we get

$$
\eta(\tau, T) \subset \mathbf{K}_{2-\mathrm{r}} \Rightarrow\left(S \cdot \tau, T^{S}\right) \in \mathbf{K}_{2-\mathrm{r}} \Rightarrow(\tau, T) \in \mathbf{K}_{2-\mathrm{r}} .
$$

In case (b) of the definition of $\eta(\tau, T)$, we get the implication $\eta(\tau, T) \subset \mathbf{K}_{2-\mathrm{r}} \Rightarrow$ $(\tau, T) \in \mathbf{K}_{2-\mathrm{r}}$, by applying Theorem 8 (b).

Finally, we consider the case (a) of the definition of $\eta(\tau, T)$. Embed $\mathcal{K}^{T}, \mathcal{Z}_{\tau} \subset \mathcal{B}_{u}$. Set $V^{\prime}=\operatorname{ker} u^{i / 2}$ and let $u^{\prime} \in \operatorname{End}\left(V^{\prime}\right)$ be the nilpotent map induced by $u$, set 
$V^{\prime \prime}=V / \operatorname{ker} u^{i / 2}$ and let $u^{\prime \prime} \in \operatorname{End}\left(V^{\prime \prime}\right)$ be the nilpotent map induced by $u$, let $\mathcal{B}_{u^{\prime}}$ and $\mathcal{B}_{u^{\prime \prime}}$ be the corresponding Springer fibers. The map

$$
\begin{aligned}
\Phi: \mathcal{B}_{u^{\prime}} \times \mathcal{B}_{u^{\prime \prime}} & \rightarrow \mathcal{B}_{u} \\
\left(V_{0}, \ldots, V_{i}\right),\left(W_{0}, \ldots, W_{n-i}\right) & \mapsto\left(V_{0}, \ldots, V_{i}, \operatorname{ker} u^{i / 2}+W_{1}, \ldots, \operatorname{ker} u^{i / 2}+W_{n-i}\right)
\end{aligned}
$$

is well defined and algebraic. Easily, we have $\Phi\left(\mathcal{B}_{u^{\prime}}^{T^{\prime}} \times \mathcal{B}_{u^{\prime \prime}}^{T^{\prime \prime}}\right) \subset \mathcal{B}_{u}^{T}$. It follows

$$
\Phi\left(\mathcal{K}^{T^{\prime}} \times \mathcal{K}^{T^{\prime \prime}}\right)=\Phi\left(\overline{\mathcal{B}_{u^{\prime}}^{T^{\prime}} \times \mathcal{B}_{u^{\prime \prime}}^{T^{\prime \prime}}}\right) \subset \mathcal{K}^{T}
$$

On the other hand, we see that $\mathcal{Z}_{\tau} \subset \Phi\left(\mathcal{Z}_{\tau^{\prime}} \times \mathcal{Z}_{\tau^{\prime \prime}}\right)$. It results:

$$
\mathcal{Z}_{\tau^{\prime}} \subset \mathcal{K}^{T^{\prime}}, \mathcal{Z}_{\tau^{\prime \prime}} \subset \mathcal{K}^{T^{\prime \prime}} \Rightarrow \mathcal{Z}_{\tau} \subset \mathcal{K}^{T}
$$

Thus, we get the implication $\eta(\tau, T) \subset \mathbf{K}_{2-\mathrm{r}} \Rightarrow(\tau, T) \in \mathbf{K}_{2-\mathrm{r}}$. The proof of the theorem is now complete.

5.3. Equivalence in the two-column case. Let $\mathbf{Y}_{2 \text {-c }}$ denote the subset of pairs $(\tau, T) \in \mathbf{Y}$ such that the Young shape $\operatorname{sh}(\tau)=\operatorname{sh}(T)$ has (at most) two columns. Set $\mathbf{K}_{2-\mathrm{c}}=\mathbf{K} \cap \mathbf{Y}_{2-\mathrm{c}}$ and $\mathbf{R}_{2-\mathrm{c}}=\mathbf{R} \cap \mathbf{Y}_{2-\mathrm{c}}$. As previously for the hook and two-row cases, we prove the equality $\mathbf{K}_{2-\mathrm{c}}=\mathbf{R}_{2-\mathrm{c}}$, and, to do this, we rely on another subset $\mathbf{A}_{2-\mathrm{c}} \subset \mathbf{Y}_{2-\mathrm{c}}$.

Let us start with some notation. Let $(\tau, T) \in \mathbf{Y}_{2-\mathrm{c}}$. We denote by $C_{q}(\tau)$ (resp. $C_{q}(T)$ ) the set of the entries in the $q$-th column of $\tau$ (resp. $T$ ). For $i \in C_{2}(\tau)$, let $\nu_{\tau}(i) \in C_{1}(\tau)$ be the entry on the left of $i$ in $\tau$. For $i \in C_{1}(\tau)$, let $\omega_{\tau}(i) \in$ $C_{2}(\tau) \cup\{\infty\}$ be either the entry on the right of $i$ in $\tau$ if there is one, or $\omega_{\tau}(i)=\infty$ otherwise. Say by convention $i<\infty$ for any integer $i$. Set $n=|\tau|=|T|$.

As in the two-row case, we define first a subset $\hat{\mathbf{A}}_{2-\mathrm{c}} \subset \mathbf{Y}_{2-\mathrm{c}}$. Recall that we denote by $\operatorname{st}(\tau)$ the standard tableau obtained by putting in increasing order the numbers inside the columns of $\tau$ (cf. 2.1). Assume $T \neq \operatorname{st}(\tau)$. Then there is $i \in\{1, \ldots, n\}$ which does not lie in the same column of $\tau$ and $T$. Take $i$ minimal. We ask for three conditions:

(1) We have $i \in C_{1}(\tau) \cap C_{2}(T)$.

(2) There is $j \in\{i+1, \ldots, n\} \cap C_{2}(\tau)$ such that $\nu_{\tau}(j) \leq i$.

Then take $j$ minimal.

(3) There is $i^{\prime} \in\{i, \ldots, j-1\} \cap C_{1}(\tau)$ such that $\omega_{\tau}\left(i^{\prime}\right)>j$.

Let $\hat{\mathbf{A}}_{2-\mathrm{c}} \subset \mathbf{Y}_{2-\mathrm{c}}$ be the subset of pairs $(\tau, T)$ such that $T \neq \operatorname{st}(\tau)$, and satisfying all the conditions (1), (2) and (3).

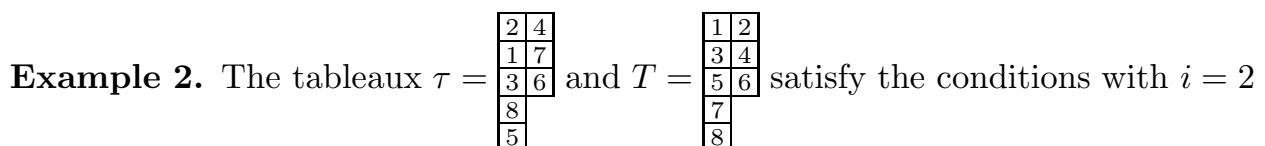
and $j=4$ and for example $i^{\prime}=3$. Thus $(\tau, T) \in \hat{\mathbf{A}}_{2-\mathrm{c}}$.

Let us now define a map $\eta: \hat{\mathbf{A}}_{2-\mathrm{c}} \rightarrow \mathbf{Y}_{2-\mathrm{c}}$. Let $(\tau, T) \in \hat{\mathbf{A}}_{2-\mathrm{c}}$, and let $i, j$ be the entries involved in conditions (1) and (2). We define a row-standard tableau $\tilde{\tau}$ with the same shape as $\tau$. We distinguish two cases.

(a) Suppose that $\omega_{\tau}(i)<\omega_{\tau}\left(i^{\prime}\right)$ for some $i^{\prime} \in\{i+1, \ldots, j-1\} \cap C_{1}(\tau)$. Take $i^{\prime}$ minimal. Then define $\tilde{\tau}$ as the tableau obtained from $\tau$ by switching $i$ and $i^{\prime}$. By condition (2), we have $\omega_{\tau}(i) \geq j$, hence $\omega_{\tau}(i)>i^{\prime}$. By definition of $i^{\prime}$ we have $\omega_{\tau}\left(i^{\prime}\right)>i$. Hence the tableau $\tilde{\tau}$ is row-standard. 
(b) Next suppose that $\omega_{\tau}\left(i^{\prime}\right) \leq \omega_{\tau}(i)$ for any $i^{\prime} \in\{i, \ldots, j-1\} \cap C_{1}(\tau)$. Then define $\tilde{\tau}$ as the tableau obtained from $\tau$ by switching $i$ and $j$. By condition (3), we have $\omega_{\tau}(i)>j$, hence the tableau $\tilde{\tau}$ is row-standard.

Then, we set $\eta(\tau, T)=(\tilde{\tau}, T)$.

Example 3. Let $\tau$ and $T$ be as in Example 2. Then $\tilde{\tau}=$\begin{tabular}{l|l|}
$\frac{3}{1}$ & 4 \\
\hline 2 & 7 \\
\hline$\frac{8}{5}$ & .
\end{tabular} .

Finally, we define the set $\mathbf{A}_{2-c}$ by induction.

- Say $(\tau, T) \in \mathbf{A}_{2-\mathrm{c}}$ whenever $T=\operatorname{st}(\tau)$.

- For $(\tau, T) \in \mathbf{Y}$ with $T \neq \operatorname{st}(\tau)$, say $(\tau, T) \in \mathbf{A}_{2-\mathrm{c}}$ if we have $(\tau, T) \in \hat{\mathbf{A}}_{2 \text {-c }}$ and $\eta(\tau, T) \in \mathbf{A}_{2-\mathrm{c}}$.

Observe that, assuming $(\tilde{\tau}, T)=\eta(\tau, T) \in \hat{\mathbf{A}}_{2-\mathrm{c}}$ and letting $\tilde{\imath} \in\{1, \ldots, n\}$ be the minimal entry which is not in the same column of $\tilde{\tau}$ and $T$, we have either $\tilde{\imath}>i$ (in case (b) of the definition of $\tilde{\tau}$ above), or $\tilde{\imath}=i$ and $\omega_{\tilde{\tau}}(\tilde{\imath})>\omega_{\tau}(i)$ (in case (a)). It follows by induction on the pair $\left(i, \omega_{\tau}(i)\right)$ that, applying $\eta$ to the pair $(\tau, T)$ a certain number of times, we obtain an element which does lie in $\hat{\mathbf{A}}_{2-\mathrm{c}}$. Therefore, the definition of the set $\mathbf{A}_{2-\mathrm{c}}$ is valid.

Example 4. Let $\tau$ and $T$ be as in Example 2, We apply $\eta$ as many times as possible:

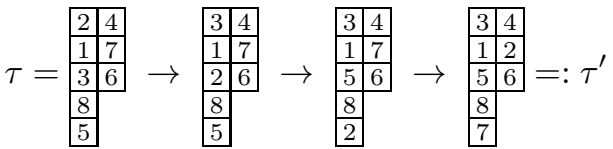

(each intermediate tableau belongs to $\hat{\mathbf{A}}_{2-\mathrm{c}}$ ). Since the last tableau satisfies $T=$ $\operatorname{st}\left(\tau^{\prime}\right)$, we get finally $(\tau, T) \in \mathbf{A}_{2-\mathrm{c}}$.

We have the following

Theorem 16. We have $\mathbf{K}_{2-\mathrm{c}}=\mathbf{A}_{2-\mathrm{c}}=\mathbf{R}_{2-\mathrm{c}}$.

Proof. The inclusion $\mathbf{K}_{2-\mathrm{c}} \subset \mathbf{R}_{2-\mathrm{c}}$ follows from Theorem 11.

Let us show the inclusion $\mathbf{A}_{2-\mathrm{c}} \subset \mathbf{K}_{2-\mathrm{c}}$. Let $(\tau, T) \in \mathbf{A}_{2-\mathrm{c}}$. If $T=\operatorname{st}(\tau)$, then we have $(\tau, T) \in \mathbf{K}_{2-\mathrm{c}}$ by Proposition 4. Assume now $(\tau, T) \in \hat{\mathbf{A}}_{2-\mathrm{c}}$. By induction, it remains to show the implication $\eta(\tau, T) \in \mathbf{K}_{2-\mathrm{c}} \Rightarrow(\tau, T) \in \mathbf{K}_{2-\mathrm{c}}$. Write $\eta(\tau, T)=$ $(\tilde{\tau}, T)$. Embed $\mathcal{K}^{T}, \mathcal{Z}_{\tau}, \mathcal{Z}_{\tilde{\tau}} \subset \mathcal{B}_{u}$. Then, it is sufficient to see that $\mathcal{Z}_{\tau}$ is contained in the closure of $\mathcal{Z}_{\tilde{\tau}}$. This follows from the Claim inside the proof of [2, Theorem $2.5]$.

Finally, let us show the inclusion $\mathbf{R}_{2-\mathrm{c}} \subset \mathbf{A}_{2-\mathrm{c}}$. Let $(\tau, T) \in \mathbf{R}_{2-\mathrm{c}}$. If $T=\operatorname{st}(\tau)$, then it is immediate that $(\tau, T) \in \mathbf{A}_{2-\mathrm{c}}$. Assume now that $T \neq \operatorname{st}(\tau)$. By induction, it is sufficient to see that $(\tau, T) \in \hat{\mathbf{A}}_{2-\mathrm{c}}$ and $\eta(\tau, T) \in \mathbf{R}_{2-\mathrm{c}}$. The fact that $(\tau, T) \in$ $\hat{\mathbf{A}}_{2-\mathrm{c}}$ is shown in the first part of the proof of [2, Theorem 2.5] (before the Claim). The fact that $\eta(\tau, T) \in \mathbf{R}_{2-\mathrm{c}}$ is proved in the last part of the proof of [2, Theorem 2.5] (after the Claim).

5.4. A connection between the two-row and two-column cases. We provide a complement to Theorem 16 where the combinatorial condition required for having $\tau \in T$ is weakened in the particular case where $\tau$ is a standard tableau (i.e. rows and also columns of $\tau$ are increasing) and $\tau, T$ have two columns: 
Proposition 17. Let $(\tau, T) \in \mathbf{Y}_{2-\mathrm{c}}$. Assume that $\tau$ is a standard tableau. Then, $\tau \in T$ if and only if $Y_{i / 0}(\tau) \preceq Y_{i / 0}^{T}$ for every $i=1, \ldots, n$.

Proof. The implication $(\Rightarrow)$ is immediate, let us show $(\Leftarrow)$. By Theorem 16, it is sufficient to derive the relation $Y_{j / i}(\tau) \preceq Y_{j / i}^{T}$ for all $0 \leq i<j \leq n$. Let $s_{j / i}^{T}$ (resp. $\left.s_{j / i}(\tau)\right)$ denote the length of the second column of $Y_{j / i}^{T}$ (resp. of $Y_{j / i}(\tau)$ ). The relation $Y_{j / i}(\tau) \preceq Y_{j / i}^{T}$ is immediate if $Y_{j / i}(\tau)$ has only one column. Otherwise, there are $a, b$ in the same row of $\tau$ such that $i<a<b \leq j$. As $\tau$ is standard, every $a^{\prime} \in C_{1}(\tau)$ with $a^{\prime} \leq a$ has a neighbor on the right $b^{\prime} \leq b$. Thus, every $a^{\prime} \in$ $\{1, \ldots, i\} \cap C_{1}(\tau)$ has a neighbor on the right $b^{\prime} \in\{1, \ldots, j\}$ in $\tau$, hence $\#\{1, \ldots, i\} \cap$ $C_{1}(\tau)$ is the number of rows of $\tau$ of the form $\left(a^{\prime}, b^{\prime}\right)$ with $a^{\prime} \leq i, a^{\prime}<b^{\prime} \leq j$. Note that $s_{j / 0}(\tau)$ (resp. $s_{j / i}(\tau)$ ) is the number of rows of $\tau$ of the form $\left(a^{\prime}, b^{\prime}\right)$ with $a^{\prime}<b^{\prime} \leq j$ (resp. with $i<a^{\prime}<b^{\prime} \leq j$ ). It follows

$$
s_{j / 0}(\tau)=s_{j / i}(\tau)+\#\{1, \ldots, i\} \cap C_{1}(\tau) .
$$

Similarly, $s_{j / 0}^{T}$ is the number of rows of length 2 in the subtableau $T[1, \ldots, j]$. The number $\#\{1, \ldots, i\} \cap C_{1}(T)$ is greater than or equal to the number of rows of the form $\left(a^{\prime}, b^{\prime}\right)$ with $a^{\prime} \leq i$ in the subtableau $T[1, \ldots, j]$. By definition of jeu de taquin, $s_{j / i}^{T}$ is greater than or equal to the number of rows of length 2 in the skew subtableau $T[i+1, \ldots, j]$. It follows

$$
s_{j / 0}^{T} \leq s_{j / i}^{T}+\#\{1, \ldots, i\} \cap C_{1}(T) .
$$

The relation $Y_{i / 0}(\tau) \preceq Y_{i / 0}^{T}$ implies $\#\{1, \ldots, i\} \cap C_{1}(\tau) \geq \#\{1, \ldots, i\} \cap C_{1}(T)$. The relation $Y_{j / 0}(\tau) \preceq Y_{j / 0}^{T}$ implies $s_{j / 0}(\tau) \leq s_{j / 0}^{T}$. Therefore, we get $s_{j / i}(\tau) \leq s_{j / i}^{T}$. It follows $Y_{j / i}(\tau) \preceq Y_{j / i}^{T}$.

Remark 3. Assume $Y(u)$ has two columns of lengths $r \geq s$. Let $\tau_{0}$ be the standard tableau of shape $Y(u)$, numbered by $1, \ldots, r$ in the first column and $r+1, \ldots, r+s$ in the second column. Then it is clear that $Y_{i / 0}\left(\tau_{0}\right) \preceq Y_{i / 0}^{T}$ for any $i$, any $T$ standard, hence $\mathcal{Z}_{\tau_{0}} \subset \mathcal{K}^{T}$ for any $T$. In particular, when $Y(u)$ has two columns, the intersection of all the components of $\mathcal{B}_{u}$ is always nonempty (see also [2, §2.1]).

We provide a connection between the two-row and two-column cases. Let $Y$ be a Young diagram. Denoting by $\lambda_{1}>\ldots>\lambda_{r}$ the lengths of the rows of $Y$, we define its transposed diagram $Y^{t}$ as the Young diagram with $\lambda_{q}$ boxes in the $q$-th column. Let $T$ be a standard tableau. We define $T^{t}$ as the standard tableau obtained by transposition: if $a_{1}<\ldots<a_{\lambda_{p}}$ are the entries of the $p$-th row of $T$, then the $p$-th column of $T^{t}$ contains $a_{1}, \ldots, a_{\lambda_{p}}$ from top to bottom.

Proposition 18. Let $T, S$ be two standard tableaux of common shape with two rows. Then we have the implication: $(S, T) \in \mathbf{K} \Rightarrow\left(T^{t}, S^{t}\right) \in \mathbf{K}$.

Proof. Assume that $(S, T) \in \mathbf{K}$. By Theorem 15, we obtain in particular the relation $Y_{i / 0}(S) \preceq Y_{i / 0}^{T}$ for any $i$. The diagrams $Y_{i / 0}(S)$ and $Y_{i / 0}^{T}$ are both defined in section 4.2 but not in the same manner. The diagrams $Y_{i / j}(S)$ and $Y_{i / j}^{S}$ may be different. Nevertheless, in the present case $j=0$, the diagrams $Y_{i / 0}(S)$ and $Y_{i / 0}^{S}$ both coincide with the shape of the subtableau $S[1, \ldots, i]$. The relation $Y_{i / 0}(S) \preceq$ $Y_{i / 0}^{T}$ implies $Y_{i / 0}\left(T^{t}\right) \preceq Y_{i / 0}^{S^{t}}$ and, as it holds for any $i$, we conclude that $\left(T^{t}, S^{t}\right) \in \mathbf{K}$ by applying Proposition 17 . 


\section{A notion of Constructible pairs $(\tau, T)$}

In the previous section, for the hook, two-row and two-column cases, we give two combinatorial characterizations of the pairs $(\tau, T)$ such that $\tau \in T$. The purpose of this section is to establish a further common combinatorial characterization, for these three cases, by transforming the inductive criterion of the previous section into an iterative one. The new criterion involves an algorithm. Starting with a pair $(\tau, T)$ in $\mathbf{Y}_{\mathrm{hk}}, \mathbf{Y}_{2-\mathrm{r}}$ or $\mathbf{Y}_{2-\mathrm{c}}$, the algorithm aims to construct the tableau $\tau$ by inserting successively $1,2,3 \ldots$ in an empty tableau, according to rules depending on $\tau$ and $T$. The algorithm either succeeds or fails to reconstruct $\tau$. We say that the pair $(\tau, T)$ is constructible if it succeeds. Then, our purpose is to show:

Theorem 19. Let $(\tau, T) \in \mathbf{Y}_{\mathrm{hk}} \cup \mathbf{Y}_{2-\mathrm{r}} \cup \mathbf{Y}_{2-\mathrm{c}}$. Then, we have $\tau \in T$ if and only if the pair $(\tau, T)$ is constructible.

We treat successively the two-row, two-column and hook cases. The algorithm in the two-row case is more simple. In section 7.3 we will use this algorithm in order to study the intersections of components in the two-row case. By presenting the algorithms in the hook and two-column cases, we want to highlight that similar iterative procedures hold also in these two cases. For the reason that we will not invoke the latter two algorithms in the subsequent sections, we will spare technical details especially in the proof of Theorem 22, regarding the two-column case.

The arguments in this section are purely combinatorial, they rely on the criteria provided in the previous section.

6.1. Constructibility in the two-row case. Fix $(\tau, T) \in \mathbf{Y}_{2-\mathrm{r}}$. We define an algorithm aiming to reconstruct $\tau$ as the final term of a sequence of tableaux $\theta_{1}, \theta_{2}, \ldots$ obtained by inserting successively the entries $1,2, \ldots$ starting with the empty tableau $\theta_{0}$. For $i \in\{0, \ldots, n\}$ the tableau $\theta_{i}$ is numbered from $1, \ldots, i$ and it has the following properties:

(2r-a) Each row of $\theta_{i}$ is a sequence of numbered boxes separated by blanks (some empty boxes). For $p \in\{1,2\}$, the entries of the $p$-th row of $\theta_{i}$ are in increasing order from left to right and they also occur in the $p$-th row of $\tau$.

(2r-b) The columns of $\theta_{i}$ being numbered from left to right, the number of entries in the $q$-th column of $\theta_{i}$ equals the number of entries $\leq i$ in the $q$-th column of $T$, for any $q \geq 1$.

For $i \in\{1, \ldots, n\}$ suppose we have constructed $\theta_{i-1}$ that satisfies properties (2r-a) and (2r-b). According to (2r-b), the number of entries in the $q$-th column of $\theta_{i-1}$ is weakly decreasing from left to right. In particular the full columns (two entries) are concentrated at the left of $\theta_{i-1}$. The tableau $\theta_{i-1}$ has the following aspect:

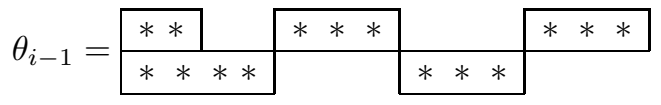

where the symbol $* * *$ stands for a strip of numbered boxes. The two strips which begin at the first column are called "in-place" strips (possibly one of the two is empty). The other strips are called "to-be-placed" strips. We form $\theta_{i}$ from $\theta_{i-1}$ by inserting $i$ according to the following rule.

(1) First case: $i$ belongs to the first row of $T$. The entry $i$ is then put in a new column on the right of $\theta_{i-1}$ in the top or bottom place depending on whether $i$ is 
in the first or in the second row of $\tau$ :

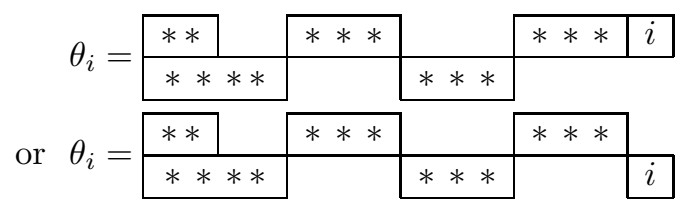

(2) Second case: $i$ belongs to the second row of $T$. Suppose $i$ lies in the $p$-th row of $\tau$, for $p \in\{1,2\}$. Then $i$ has to be inserted in the $p$-th row of $\theta_{i-1}$. We state this failure case:

(Failure case) (With $i$ in the second row of $T$ and in the $p$-th row of $\tau$.) If the $p$-th box of the last column of $\theta_{i-1}$ is nonempty, then the algorithm fails.

Suppose this failure case does not arise. Then, the entry $i$ is put at the end of the last strip of the $p$-th row. Next, from right to left, the first entries of the "to-beplaced" strips are successively pushed to the left at the end of the preceding strip (at the beginning of the row if there is no preceding strip):

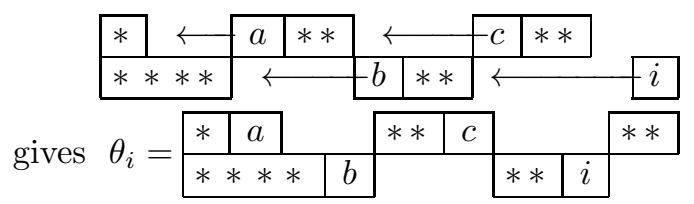

If no failure case occurs while $i$ runs over $\{1, \ldots, n\}$, then we get a final tableau $\theta_{n}$ with entries $1, \ldots, n$. According to $(2 \mathrm{r}-\mathrm{a})$ and $(2 \mathrm{r}-\mathrm{b})$ we have $\theta_{n}=\tau$. Then we say that the pair $(\tau, T)$ is constructible.

Example 5. Let $T=$\begin{tabular}{|l|l|l|l|l|}
\hline 1 & 2 & 3 & 4 & 7 \\
\hline 5 & 6 & 8 & 9 & .
\end{tabular} .

(a) First, we suppose $\tau=$\begin{tabular}{|l|l|l|l|l|}
\hline 2 & 3 & 6 & 8 & 9 \\
\hline 1 & 4 & 5 & 7 & .
\end{tabular} .

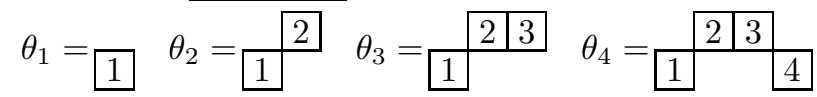

The number 5 is in the second row of $T$ and in the second row of $\tau$. The second box of the last column of $\theta_{4}$ is not empty. Due to the failure rule, the algorithm fails.

(b) Suppose now $\tau=$\begin{tabular}{|l|l|l|l|l|}
\hline 1 & 4 & 6 & 8 & 9 \\
\hline 2 & 3 & 5 & 7 & .
\end{tabular} .

Likewise

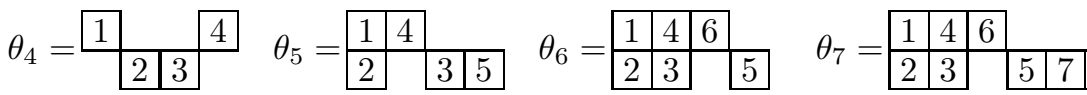

$$
\begin{aligned}
& \theta_{8}=\begin{array}{l|l|l|l|l}
\hline & 4 & 6 & 8 \\
\hline 2 & 3 & 5 & & 7
\end{array} \quad \text { and finally } \theta_{9}=\begin{array}{ll|l|l|l|l}
1 & 4 & 6 & 8 & 9 \\
2 & 3 & 5 & 7
\end{array}=\tau .
\end{aligned}
$$

Thus in this example $(\tau, T)$ is constructible.

Let $\theta_{1}, \theta_{2}, \ldots$ be the intermediate tableaux obtained by applying the algorithm to the pair $(\tau, T)$. (Implicitly, no fail has occurred while $\theta_{i}$ is well defined.) Each tableau $\theta_{i}$ is formed by strips that we number in the following manner:

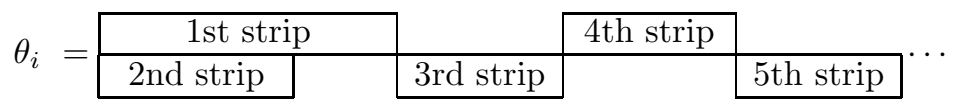


or possibly

$$
\begin{array}{l|l|l|l|l|}
\theta_{i}= & \text { 1st strip } & \text { 3rd strip } & & \text { 5th strip } \\
\hline \text { 2nd strip } & & \text { 4th strip } &
\end{array}
$$

The first two strips are left-justified, possibly one of the two is empty. We denote by $s_{1}\left(\theta_{i}\right)$ (resp. by $s_{2}\left(\theta_{i}\right)$ ) the length of the first strip (resp. the second strip) of $\theta_{i}$. We show the following

Lemma 20. Suppose that there are $i, j$ such that $s_{1}\left(\theta_{i}\right)<s_{2}\left(\theta_{i}\right)$ and $s_{1}\left(\theta_{j}\right)>$ $s_{2}\left(\theta_{j}\right)$, then the pair $(\tau, T)$ satisfies condition $5.2(1)$.

Proof. By the construction of $\theta_{k}$ from $\theta_{k-1}$, we see that, for $p \in\{1,2\}$, either $s_{p}\left(\theta_{k}\right)=s_{p}\left(\theta_{k-1}\right)$ or $s_{p}\left(\theta_{k}\right)=s_{p}\left(\theta_{k-1}\right)+1$. Thus we always have

$$
\left(s_{1}\left(\theta_{k}\right)-s_{2}\left(\theta_{k}\right)\right)-\left(s_{1}\left(\theta_{k-1}\right)-s_{2}\left(\theta_{k-1}\right)\right) \in\{-1,0,1\} .
$$

Since $s_{1}\left(\theta_{i}\right)-s_{2}\left(\theta_{i}\right)<0$ and $s_{1}\left(\theta_{j}\right)-s_{2}\left(\theta_{j}\right)>0$, there is $k \in\{\min (i, j)+$ $1, \ldots, \max (i, j)-1\}$ such that $s_{1}\left(\theta_{k}\right)-s_{2}\left(\theta_{k}\right)=0$. The equality $s_{1}\left(\theta_{k}\right)=s_{2}\left(\theta_{k}\right)$ implies that, starting with the third one, all the strips of $\theta_{k}$ are empty. Then the shape of $\theta_{k}$ is a rectangular Young diagram with two rows of length $k / 2$ and, according to $(2 \mathrm{r}-\mathrm{a})$ and $(2 \mathrm{r}-\mathrm{b})$, this is also the shape of the subtableaux $\tau[1, \ldots, k]$ and $T[1, \ldots, k]$. Therefore, the pair $(\tau, T)$ satisfies condition $5.2(1)$.

We prove the following result.

Theorem 21. Let $(\tau, T) \in \mathbf{Y}_{2-\mathrm{r}}$. Then, we have $\tau \in T$ if and only if the pair $(\tau, T)$ is constructible.

Proof. Write $n=|\tau|=|T|$. First, let us show that, if the pair $(\tau, T)$ is constructible, then we have $(\tau, T) \in \hat{\mathbf{A}}_{2-\mathrm{r}}$. To do this, we have to show that $(\tau, T)$ satisfies either 5.2 (1) or $5.2(2)$. If $5.2(2)$ does not hold, then 1 is in the second row of $\tau$ or there is $i \in\{2, \ldots, n\}$ such that $T[1, \ldots, i]$ is rectangular. In the former case $\theta_{1}={ }_{1}^{\cdot}$, which gives $s_{1}\left(\theta_{1}\right)=0$ and $s_{2}\left(\theta_{1}\right)=1$. Since $(\tau, T)$ is constructible, we have $\theta_{n}=\tau$, hence $s_{1}\left(\theta_{n}\right) \geq s_{2}\left(\theta_{n}\right)$. Then, by Lemma 20, the pair $(\tau, T)$ satisfies condition $5.2(1)$. In the latter case it follows from $(2 \mathrm{r}-\mathrm{b})$ that $\theta_{i}$ has a rectangular shape and it follows from $(2 \mathrm{r}-\mathrm{a})$ that $\theta_{i}$ coincides with the subtableau $\tau[1, \ldots, i]$. Thus $T[1, \ldots, i]$ and $\tau[1, \ldots, i]$ are both rectangular and condition $5.2(1)$ holds. Therefore, $(\tau, T)$ satisfies $5.2(1)$ or $5.2(2)$.

By Theorem 15 and by definition of the set $\mathbf{A}_{2-\mathrm{r}}$, in order to prove the theorem, it is sufficient to show that any $(\tau, T) \in \hat{\mathbf{A}}_{2 \text {-r }}$ is constructible if and only if the pair or the two pairs forming $\eta(\tau, T)$ are constructible. We distinguish three cases, corresponding to the three cases of the construction of $\eta(\tau, T)$ in 5.2 .

(a) Suppose that there is $i<n$ such that $\tau[1, \ldots, i]$ and $T[1, \ldots, i]$ are both rectangular, and choose $i$ maximal for this property.

Then, let $\eta(\tau, T)=\left\{\left(\tau^{\prime}, T^{\prime}\right),\left(\tau^{\prime \prime}, T^{\prime \prime}\right)\right\}$ be defined as in 5.2 (a). Let $\theta_{1}, \theta_{2}, \ldots$ (resp. $\left.\theta_{1}^{\prime}, \theta_{2}^{\prime}, \ldots\right)$ (resp. $\theta_{1}^{\prime \prime}, \theta_{2}^{\prime \prime}, \ldots$ ) be the tableaux obtained by applying the algorithm to the pair $(\tau, T)$ (resp. $\left.\left(\tau^{\prime}, T^{\prime}\right)\right)$ (resp. $\left.\left(\tau^{\prime \prime}, T^{\prime \prime}\right)\right)$. It immediately follows from the definition of the algorithm that $\theta_{j}=\theta_{j}^{\prime}$ for $j \leq i$. Therefore $\left(\tau^{\prime}, T^{\prime}\right)$ is constructible if and only if the algorithm for $(\tau, T)$ does not fail in the first $i$ steps, and in this case $\theta_{i}$ coincides with the tableau $\tau^{\prime}$. Assume that $\left(\tau^{\prime}, T^{\prime}\right)$ is constructible. It follows from the definition of the algorithm that, for $j \geq i+1$, the tableau $\theta_{i}$ is obtained 
by adding $\theta_{j-i}^{\prime \prime}$ on the right of $\tau^{\prime}$ and replacing every entry $k$ in $\theta_{j-i}^{\prime \prime}$ by $k+i$. Thus, it is easy to see that $\left(\tau^{\prime \prime}, T^{\prime \prime}\right)$ is constructible if and only if the algorithm for $(\tau, T)$ does not fail also until the end. Finally, we get that $(\tau, T)$ is constructible if and only if both $\left(\tau^{\prime}, T^{\prime}\right)$ and $\left(\tau^{\prime \prime}, T^{\prime \prime}\right)$ are constructible.

(b) Suppose that $\tau[1, \ldots, i]$ and $T[1, \ldots, i]$ are both rectangular only for $i=n$.

If $\tilde{\tau}$ denotes the tableau obtained by switching the two rows of $\tau$, then it is immediate from the definition of the algorithm that $(\tau, T)$ is constructible if and only if $(\tilde{\tau}, T)$ is constructible. Up to dealing with $\tilde{\tau}$ instead of $\tau$, we may suppose that $n$ is the last entry in the second row of $\tau$. Then $\eta(\tau, T)=\left\{\left(\tau^{\prime}, T^{\prime}\right)\right\}$ with $\tau^{\prime}=\tau[1, \ldots, n-1]$ and $T^{\prime}=T[1, \ldots, n-1]$. Let $\theta_{1}, \theta_{2}, \ldots\left(\operatorname{resp} . \theta_{1}^{\prime}, \theta_{2}^{\prime}, \ldots\right)$ be the tableaux obtained by applying the algorithm to the pair $(\tau, T)\left(\operatorname{resp} .\left(\tau^{\prime}, T^{\prime}\right)\right)$. It follows from the definition of the algorithm that we have $\theta_{i}=\theta_{i}^{\prime}$ for $i \leq n-1$. Thus, $\left(\tau^{\prime}, T^{\prime}\right)$ is constructible if and only if the algorithm for $(\tau, T)$ does not fail in the first $n-1$ steps, and in this case $\theta_{n-1}=\tau^{\prime}$. In the last step of the algorithm relative to the pair $(\tau, T)$, the entry $n$ has to be inserted in the second row of $\theta_{n-1}$. The last box in the second row of $\theta_{n-1}=\tau^{\prime}$ is empty, hence the algorithm does not fail in the $n$-th step whenever it has not failed before. Finally, we get that $(\tau, T)$ is constructible if and only if $\left(\tau^{\prime}, T^{\prime}\right)$ is constructible.

(c) Finally, suppose that $5.2(2)$ holds.

Let $\eta(\tau, T)=\left(\tau^{\prime}, T^{\prime}\right)$ be as in $5.2(\mathrm{c})$. Let $\theta_{1}, \theta_{2}, \ldots$ (resp. $\left.\theta_{1}^{\prime}, \theta_{2}^{\prime}, \ldots\right)$ be the tableaux obtained by applying the algorithm to the pair $(\tau, T)\left(\operatorname{resp} .\left(\tau^{\prime}, T^{\prime}\right)\right)$. By hypothesis, 1 lies in the first row of $\tau$, hence $s_{1}\left(\theta_{1}\right)=1$ and $s_{2}\left(\theta_{1}\right)=0$. By Lemma 20. we get $s_{1}\left(\theta_{i}\right)>s_{2}\left(\theta_{i}\right)$ for any $i$. Thus $\theta_{i}$ has the following form:

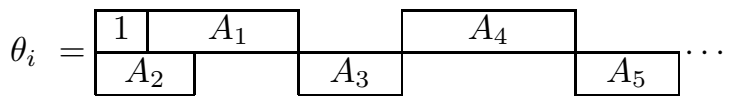

with $\# A_{1} \geq \# A_{2}$, where $\# A$ denotes the number of boxes in $A$. Let $\tilde{\theta}_{i}$ be the tableau obtained by removing 1 and moving $A_{1}, A_{3}, A_{4}, \ldots$ by one rank to the left:

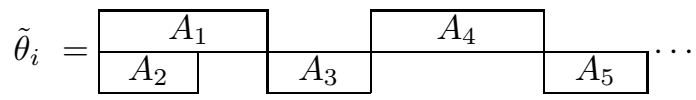

Possibly the gap between $A_{2}$ and $A_{3}$ in $\tilde{\theta}_{i}$ is empty. The tableau $\tilde{\theta}_{i}$ is numbered by $2, \ldots, i$. Let us show the following claim.

Claim. Let $i \in\{2, \ldots, n\}$. The algorithm relative to $(\tau, T)$ does not fail in the first $i$ steps if and only if the one relative to $\left(\tau^{\prime}, T^{\prime}\right)$ does not fail in the first $i-1$ steps. Moreover, the tableau $\theta_{i-1}^{\prime}$ is obtained from $\tilde{\theta}_{i}$ by replacing every entry $j$ by $j-1$.

The proof of the claim ends the proof of the Theorem, because it implies in particular that $(\tau, T)$ is constructible if and only if $\left(\tau^{\prime}, T^{\prime}\right)$ is.

We show the claim by induction on $i \geq 2$. Condition $5.2(2)$ implies in particular that 2 lies in the first row of $T$, hence the algorithm for $(\tau, T)$ does not fail in the first two steps, and the claim is immediate for $i=2$. Suppose the claim holds until $i-1 \in\{2, \ldots, n-1\}$ and show it for $i$. The row number of $i$ in $T$ and the row number of $i-1$ in $T^{\prime}$ coincide. Moreover, the row number of $i$ in $\tau$ and the row number of $i-1$ in $\tau^{\prime}$ are the same, say $p$. Assume that $i$ lies in the first row of $T$, then $i-1$ is in the first row of $T^{\prime}$ and both algorithms do not fail. Now, assume that $i$ lies in the second row of $T$. The tableau $T[1, \ldots, i]$ being non-rectangular, it follows from (2r-b) that also $\tilde{\theta}_{i-1}$ is non-rectangular, thus the last columns of $\theta_{i-1}$ and $\tilde{\theta}_{i-1}$ 
coincide. By induction hypothesis, we get that the $p$-th box of the last column of $\theta_{i-1}$ is empty if and only if the $p$-th box of the last column of $\theta_{i-2}^{\prime}$ is empty. Thus the algorithm for $(\tau, T)$ fails at the $i$-th step if and only if the algorithm for $\left(\tau^{\prime}, T^{\prime}\right)$ fails at the $(i-1)$-th step. In each case, assuming that both algorithms do not fail, the fact that $\theta_{i-1}^{\prime}$ coincides with $\tilde{\theta}_{i}$ up to replacing each entry $j$ by $j-1$ follows by induction hypothesis and the definition of the algorithm.

6.2. Constructibility in the two-column case. Let $(\tau, T) \in \mathbf{Y}_{2-\mathrm{c}}$. Write $n=$ $|\tau|=|T|$. Let $r$ be the common length of the first column of $\tau, T$. Let $Y$ be the Young diagram with two columns of $r$ empty boxes

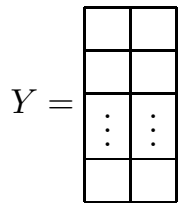

The algorithm which we define aims to reconstruct $\tau$ as the final term of a sequence of tableaux $\theta_{1}, \theta_{2}, \ldots$ obtained by inserting successively the entries $1,2, \ldots$ in the diagram $Y$.

We consider tableaux which are partial numberings of $Y$. The tableaux $\tau$ and $T$ and their subtableaux $\tau[i, \ldots, j]$ and $T[i, \ldots, j]$ of entries $i, \ldots, j$, for any integers $1 \leq i \leq j \leq n$, are considered as partial numberings of $Y$. Let $\theta_{0}$ be the empty numbering of $Y$. The tableau $\theta_{i}$ obtained at the $i$-th step of the algorithm is a partial numbering of entries $1, \ldots, i$. Set $\overline{\mathbb{N}}=\{0,1,2, \ldots\} \cup\{\infty\}$. By convention $\infty=\infty+1=\infty-1$ and $a<\infty$ for any $a \in \mathbb{N}$. Let $P=\{1, \ldots, r\}$ be the rows of $Y$ numbered from top to bottom. For each step $i=1,2, \ldots$ of the algorithm and for each row $p \in P$ we define in addition an index $f_{i}(p) \in \overline{\mathbb{N}}$. As an initialization, we set $f_{0}(p)=\infty$ for any $p \in P$.

For $i \in\{1, \ldots, n\}$ the tableau $\theta_{i}$ has the following properties:

(2c-a) For $p \in P$ the entries of the $p$-th row of $\theta_{i}$ are in increasing order to the right and they also occur in the $p$-th row of $\tau$.

(2c-b) For $q \in\{1,2\}$ the number of entries in the $q$-th column of $\theta_{i}$ is equal to the number of entries $\leq i$ in the $q$-th column of $T$.

(2c-c) Let $P_{i} \subset P$ be the subset of rows of $\theta_{i}$ whose first box is nonempty and let $Q_{i} \subset P$ be the subset of the other non-empty rows. For $p \in P$ we have $f_{i}(p) \in \mathbb{N}$ if and only if $p \in P_{i}$. In addition, $\max _{p \in P_{i}} f_{i}(p)=\# Q_{i}$.

For $i \in\{1, \ldots, n\}$ suppose that we have constructed $\theta_{i-1}$ that satisfies these properties. For instance, $\theta_{i-1}$ has the following aspect:

$$
\theta_{i-1}=\begin{array}{|l|l|l}
\hline & * & \infty \\
\hline * & * & f_{i-1}(2) \\
\hline & & \infty \\
& & f_{i-1}(4) \\
& & f_{i-1}(5) \\
\hline
\end{array}
$$

The symbol $*$ stands for a numbered box. We have written the values $f_{i-1}(p)$ at the right. In this example we have $P_{i-1}=\{2,4,5\}$ and $Q_{i-1}=\{1\}$.

We form $\theta_{i}$ from $\theta_{i-1}$ by inserting $i$ according to the following rules.

(0) Let $p_{i} \in P$ be the number of the row of $\tau$ to which $i$ belongs. The entry $i$ has to be put in the $p_{i}$-th row of $\theta_{i-1}$. We state this failure case: 
(First failure case) If the second box of the $p_{i}$-th row of $\theta_{i-1}$ is not empty, then the algorithm fails.

Suppose that no failure of this type arises. Then put $i$ in the second box of the $p_{i}$-th row of $\theta_{i-1}$. Let $\theta_{i}^{\prime}$ be the tableau so-obtained.

(1) First case: $i$ belongs to the second column of $T$. Then define $\theta_{i}=\theta_{i}^{\prime}$. Let $p \in P$. If $f_{i-1}(p)<f_{i-1}\left(p_{i}\right)$, then set $f_{i}(p)=f_{i-1}(p)+1$. If $f_{i-1}(p) \geq f_{i-1}\left(p_{i}\right)$, then set $f_{i}(p)=f_{i-1}(p)$.

For $\theta_{i-1}$ as in the previous figure, we could have for example

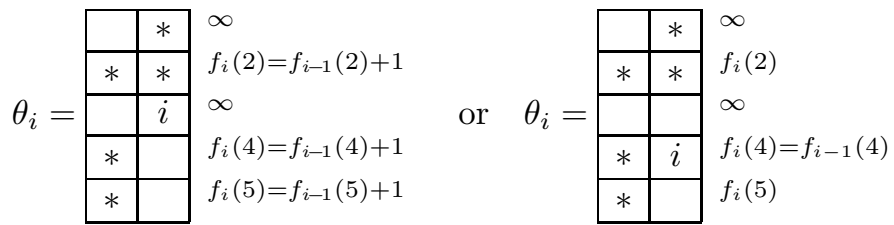

In the case of the first tableau, the finite indices have been incremented. In the case of the second tableau, for $p \in\{2,5\}$, we have $f_{i}(p)=f_{i-1}(p)$ or $f_{i}(p)=f_{i-1}(p)+1$ depending on whether $f_{i-1}(p) \geq f_{i-1}(4)$ or $f_{i-1}(p)<f_{i-1}(4)$.

Properties (2c-a), (2c-b), (2c-c) hold easily for $\theta_{i}$.

(2) Second case: $i$ belongs to the first column of $T$. We construct $\theta_{i}$ from the intermediate tableau $\theta_{i}^{\prime}$ by pushing in addition one entry to the left. We state:

(Second failure case) Suppose that $i$ is in the first column of $T$. If $f_{i-1}\left(p_{i}\right)=0$, then the algorithm fails.

Suppose no failure arises. Let $F_{i}^{\prime}$ be the set of the entries of the second column of $\theta_{i}^{\prime}$ which have an empty box on their left. The set $F_{i}^{\prime}$ is nonempty. (Indeed, we have either $f_{i-1}\left(p_{i}\right)=\infty$ or $f_{i-1}\left(p_{i}\right) \in \mathbb{N} \backslash\{0\}$. In the former case, $i \in F_{i}^{\prime}$. In the latter case, due to $(2 \mathrm{c}-\mathrm{c}), Q_{i-1} \neq \emptyset$, hence also $F_{i}^{\prime} \neq \emptyset$.) By (2c-a) each $j \in F_{i}^{\prime}$ belongs to the first column of $\tau$. If possible, choose $j \in F_{i}^{\prime}$ with a minimal right-neighbor entry in $\tau$. If no element of $F_{i}^{\prime}$ has a right-neighbor entry in $\tau$, then choose $j \in F_{i}^{\prime}$ with $p_{j}$ minimal. Let $\theta_{i}$ be the tableau obtained from $\theta_{i}^{\prime}$ by pushing $j$ by one rank to the left.

We set $f_{i}\left(p_{j}\right)=0$. Let $p \in P$ be such that $p \neq p_{j}$. If $f_{i-1}(p)<f_{i-1}\left(p_{i}\right)$, then set $f_{i}(p)=f_{i-1}(p)$. If $f_{i-1}(p) \geq f_{i-1}\left(p_{i}\right)$, then set $f_{i}(p)=f_{i-1}(p)-1$.

For $\theta_{i-1}$ as in the previous figure, we get for example:
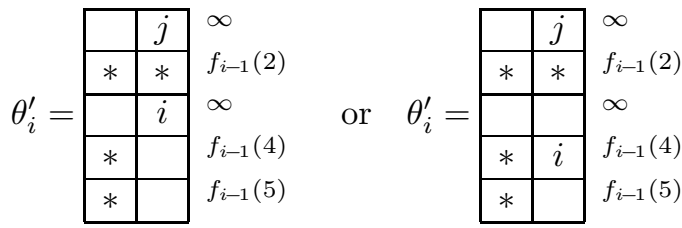

and one entry has yet to be moved to the left. In the case of the first tableau, no failure can occur, since $f_{i-1}\left(p_{i}\right)=\infty$. We have $F_{i}^{\prime}=\{i, j\}$. We move $i$ or $j$ to the left. If one of them has an entry on its right in $\tau$ we move the one for which this is minimal. Otherwise we move $j$, since the row-number of $j$ is smaller. As $f_{i-1}\left(p_{i}\right)=\infty$, the indices do not change. In the case of the second tableau, a failure occurs if and only if $f_{i-1}(4)=0$. If no failure occurs, then $j$ is moved to the left and we set $f_{i}(1)=0$. The other indices which are greater or equal than $f_{i-1}\left(p_{i}\right)$ are reduced by one unit.

Easily properties (2c-a), (2c-b) and (2c-c) hold for $\theta_{i}$. 
If no failure case occurs while $i$ runs over $\{1, \ldots, n\}$, then we get a final tableau $\theta_{n}$ with entries $1, \ldots, n$. According to $(2 \mathrm{c}-\mathrm{a})$ and $(2 \mathrm{c}-\mathrm{b})$ we have $\theta_{n}=\tau$. Then we say that the pair $(\tau, T)$ is constructible.

Example 6. (a) Let $\tau$ and $T$ be the tableaux

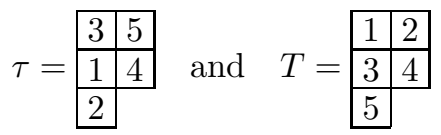

We get the following $\theta_{1}, \ldots, \theta_{5}$. We write the indices $f_{i}(p)$ with roman numerals at the right.
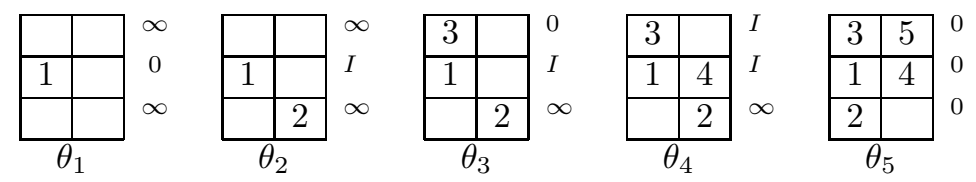

No failure has occurred and the pair $(\tau, T)$ is thus constructible.

(b) Suppose that $\tau$ and $T$ are the tableaux

$$
\tau=\begin{array}{|l|l|}
\hline 2 & 6 \\
\hline 3 & 5 \\
\cline { 1 - 1 } 4 & \\
\cline { 1 - 1 } 1 &
\end{array} \quad \text { and } \quad T=\begin{array}{|l|l|}
\hline 1 & 2 \\
\hline 3 & 4 \\
\hline 5 & \\
\hline 6 &
\end{array}
$$
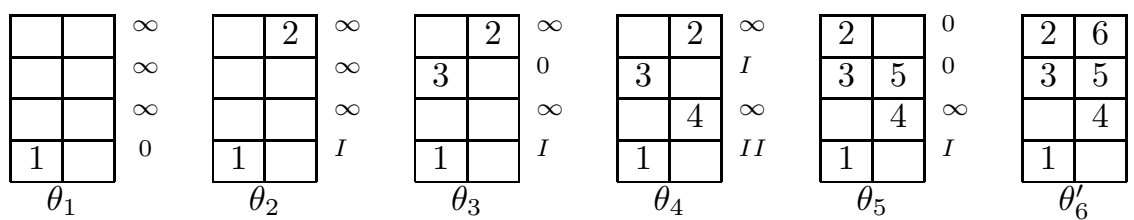

The entry 6 is inserted in the first row of $\theta_{5}$. In addition 6 belongs to the second row of $T$ hence one entry has to be moved to the left. But we have $f_{5}(1)=0$, hence we are in the second failure case. Finally $(\tau, T)$ is not constructible.

We have the following result.

Theorem 22. Let $(\tau, T) \in \mathbf{Y}_{2-\mathrm{c}}$. Then, we have $\tau \in T$ if and only if the pair $(\tau, T)$ is constructible.

The proof of Theorem 22 requires many technical verifications. For the sake of conciseness, we will underline the main steps of the proof without going into details. The proof relies on the set $\mathbf{A}_{2 \text {-c }}$ introduced in section 5.3. Recall that $\operatorname{st}(\tau)$ is the standard tableau obtained by putting each column of $\tau$ in increasing order. Considering the inductive definition of the set $\mathbf{A}_{2-c}$ and applying Theorem 16. Theorem 22 is a consequence of the following

Proposition 23. Let $(\tau, T) \in \mathbf{Y}_{2-\mathrm{c}}$.

(a) If $T=\operatorname{st}(\tau)$, then the pair $(\tau, T)$ is constructible.

(b) If $(\tau, T)$ is constructible, then we have $T=\operatorname{st}(\tau)$ or $(\tau, T) \in \hat{\mathbf{A}}_{2-\mathrm{c}}$.

(c) Assume $(\tau, T) \in \hat{\mathbf{A}}_{2-\mathrm{c}}$. Then the pair $(\tau, T)$ is constructible if and only if $\eta(\tau, T)$ is constructible.

Proposition 23 (a) is a consequence of the following lemma, which easily follows, by induction, from the definition of the algorithm. 
Lemma 24. Let $k \in\{1, \ldots, n\}$. If each $l \in\{1, \ldots, k\}$ is in the same column in $\tau$ and $T$, then the algorithm does not fail in $\{1, \ldots, k\}$. In addition $\theta_{k}$ coincides with the subtableau $\tau[1, \ldots, k]$ of entries $1, \ldots, k$, and we have $f_{k}(p)=0$ for all $p \in P_{k}$.

The next goal to show Proposition 23(b) requires two preliminary observations. Let $(\tau, T) \in \mathbf{Y}_{2-\mathrm{c}}$ be such that $T \neq \operatorname{st}(\tau)$. Take $i \in\{1, \ldots, n\}$ minimal which does not lie in the same column in $\tau$ and $T$. By Lemma 24, the algorithm does not fail in the first $i-1$ steps. Recall that $C_{q}(\tau)$ (resp. $C_{q}(T)$ ) is the set of entries in the $q$-th column of $\tau$ (resp. of $T$ ). By Lemma 24 and the second failure case, we have:

Lemma 25. If the algorithm does not fail at the $i$-th step, then $i \in C_{1}(\tau) \cap C_{2}(T)$.

Let $\nu_{\tau}: C_{2}(\tau) \rightarrow C_{1}(\tau)$ and $\omega_{\tau}: C_{1}(\tau) \rightarrow C_{2}(\tau) \cup\{\infty\}$ be the maps introduced in section 5.3. The sets $P_{k}, Q_{k}$ are those involved in condition (2c-c). We need the following technical

Lemma 26. Let $k \geq i$ be such that $\nu_{\tau}(l)>i$ for any $l \in\{i+1, \ldots, k\} \cap C_{2}(\tau)$. Suppose that the algorithm does not fail in the first $k$ steps. Then the set $Q_{k}$ is nonempty and we have $f_{k}(p)<\# Q_{k}=f_{k}\left(p^{\prime}\right)$ for any $p \in P_{k} \backslash P_{i}$ and $p^{\prime} \in P_{i}$.

We show Proposition 23(b): assume that the pair $(\tau, T), T \neq \operatorname{st}(\tau)$ is constructible, and let us show that $(\tau, T) \in \hat{\mathbf{A}}_{2-\mathrm{c}}$. Lemma 25 exactly means that $(\tau, T)$ satisfies condition $5.3(1)$. As $(\tau, T)$ is constructible, $Q_{n}=\emptyset$. Then, by Lemma 26. there is $j \in\{i+1, \ldots, n\} \cap C_{2}(\tau)$ such that $\nu_{\tau}(j) \leq i$. This is condition 5.3(2). Take $j$ minimal for this property. Also by Lemma [26, $Q_{j-1} \neq \emptyset$. Hence there is $i^{\prime} \in\{i, \ldots, j-1\} \cap C_{1}(\tau)$ which is in the second column of $\theta_{j-1}$. This implies that $\omega_{\tau}\left(i^{\prime}\right)>j$. Condition $\left[5.3(3)\right.$ is thus satisfied. Finally, we get $(\tau, T) \in \hat{\mathbf{A}}_{2-\mathrm{c}}$.

It remains to show Proposition 23 (c). Suppose $(\tau, T) \in \hat{\mathbf{A}}_{2-\mathrm{c}}$. Let $j>i$ be the integers involved in 5.3(1)-(3). Recall that the pair $\eta(\tau, T)=(\tilde{\tau}, T)$ is defined in section 5.3. according to the following rule:

(a) If there is $i^{\prime} \in\{i+1, \ldots, j-1\} \cap C_{1}(\tau)$ such that $\omega_{\tau}(i)<\omega_{\tau}\left(i^{\prime}\right)$, then take $i^{\prime}$ minimal for this property and $\tilde{\tau}$ is obtained from $\tau$ by switching $i$ and $i^{\prime}$.

(b) Otherwise, $\tilde{\tau}$ is obtained from $\tau$ by switching $i$ and $j$.

We set $\tilde{\imath}=i^{\prime}$ in case (a) and $\tilde{\imath}=j$ in case (b), so that the tableau $\tilde{\tau}$ is obtained from $\tau$ by switching $i$ and $\tilde{\imath}$. Let $\tilde{p}_{j}, \tilde{\theta}_{j}, \tilde{f}_{j}, \tilde{P}_{j}, \tilde{Q}_{j}$ be the analogues of $p_{j}, \theta_{j}, f_{j}, P_{j}, Q_{j}$ for the pair $(\tilde{\tau}, T)$. By Lemma 24, the algorithms relative to $(\tau, T)$ and $(\tilde{\tau}, T)$ do not fail in the first $i-1$ steps. The following lemma compares both algorithms from the $i$-th step. It can be proved by induction. The proof, which we skip, consists of technical verifications, relying on the definition of the algorithm and on Lemma 26 as another ingredient.

Lemma 27. Let $k \in\{i, \ldots, \tilde{\imath}\}$.

(a) The algorithm relative to the tableau $\tau$ does not fail in the first $k$ steps if and only if the algorithm relative to $\tilde{\tau}$ does not.

Suppose now that both algorithms do not fail in the first $k$ steps.

(b) Case $i \leq k<\tilde{i}$. The entries of $\theta_{k}$ and $\tilde{\theta}_{k}$ have the same place in both tableaux except $i$ which is in the second box of the $p_{i}$-th row of $\theta_{k}$ and in the second box of the $p_{\tilde{\imath}}$-th row of $\tilde{\theta}_{k}$. We have $f_{k}(p)=\tilde{f}_{k}(p) \forall p \notin P_{i}$.

(c) Case $k=\tilde{\imath}$. The tableaux $\theta_{\tilde{\imath}}$ and $\tilde{\theta}_{\tilde{\imath}}$ are obtained one from the other by switching $i$ and $\tilde{\imath}$. We have $f_{\tilde{\imath}}(p)=\tilde{f}_{\tilde{\imath}}(p) \forall p \in P$. 
Proposition 23(c) then follows from Lemma 27 we have to show that the algorithm relative to the pair $(\tau, T)$ succeeds if and only if the algorithm relative to the pair $(\tilde{\tau}, T)$ succeeds. By Lemma 27(a), we may assume that both algorithms have not failed in the first $\tilde{\imath}$ steps. It is easy to see that the success of the algorithm after the $\tilde{\imath}$-th step only depends on the shape of $\theta_{\tilde{\imath}}$, on the values of the map $f_{\tilde{\imath}}$ and on the subtableau $\tau[\tilde{\imath}+1, \ldots, n]$ of entries $\tilde{\imath}+1, \ldots, n$. By Lemma 27(c), the tableaux $\theta_{\tilde{\imath}}$ and $\tilde{\theta}_{\tilde{\imath}}$ have the same shape, the maps $f_{\tilde{\imath}}$ and $\tilde{f}_{\tilde{\imath}}$ are equal, whereas the subtableaux $\tau[\tilde{\imath}+1, \ldots, n]$ and $\tilde{\tau}[\tilde{\imath}+1, \ldots, n]$ coincide. Therefore, both algorithms fail or succeed simultaneously.

6.3. Constructibility in the hook case. Let $(\tau, T) \in \mathbf{Y}_{\mathrm{hk}}$. Set $n=|\tau|=|T|$. Let $r$ be the common length of the first column of $\tau, T$ and let $s$ be the length of their first row. Let $Y$ be the rectangular Young diagram with $s+1$ columns of length $r$ (the $(s+1)$-th column may be implicit)

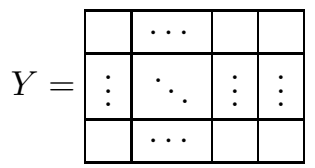

The algorithm which we define aims to reconstruct $\tau$ as the final term of a sequence of tableaux $\theta_{1}, \theta_{2}, \ldots$ obtained by inserting successively the entries $1,2, \ldots$ in the diagram $Y$.

We consider tableaux which are partial numberings of $Y$. The tableaux $\tau$ and $T$ and their subtableaux $\tau[i, \ldots, j]$ and $T[i, \ldots, j]$ are considered as partial numberings of $Y$. Let $\theta_{0}$ be the empty numbering of $Y$. The tableau $\theta_{i}$ obtained at the $i$-th step of the algorithm is a partial numbering of entries $1, \ldots, i$, and it has the following properties:

(h-a) For $p \in\{1, \ldots, r\}$, the entries of the $p$-th row of $\theta_{i}$ belong to the $p$-th row of $\tau$. The entries of the first row of $\theta_{i}$ are in increasing order.

(h-b) For $q \in\{1, \ldots, s\}$, the number of entries in the $q$-th column of $\theta_{i}$ equals the number of entries $\leq i$ in the $q$-th column of $T$.

(h-c) $\theta_{i}$ and $\tau[1, \ldots, i]$ differ by at most one entry, whose row number is $\geq 2$.

For $i \in\{1, \ldots, n\}$ suppose that we have constructed $\theta_{i-1}$ satisfying these properties. Let $s_{i-1} \in\{1, \ldots, s\}$ be the length of the first row of the subtableau $\tau[1, \ldots, i-1]$. Properties (h-a), (h-b) and (h-c) imply that $\theta_{i-1}$ has the following aspect:
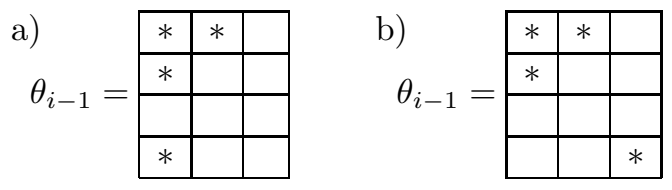

a) either the tableau $\theta_{i-1}$ and $\tau[1, \ldots, i-1]$ coincide, then the $\left(s_{i-1}+1\right)$-th column of $\theta_{i-1}$ is empty,

b) or they differ by exactly one entry $j$ which has the same row number $\geq 2$ in both tableaux, but $j$ is in the first column of $\tau[1, \ldots, i-1]$ and the unique entry in the $\left(s_{i-1}+1\right)$-th column of $\theta_{i-1}$.

We construct $\theta_{i}$ from $\theta_{i-1}$ in the following manner.

(0) Let $p \in\{1, \ldots, r\}$ be the row number of $i$ in $\tau$. Note that the $p$-th box of the $\left(s_{i-1}+1\right)$-th column of $\theta_{i-1}$ is empty. Put $i$ in this box and denote by $\theta_{i}^{\prime}$ the tableau so-obtained. 
For instance:
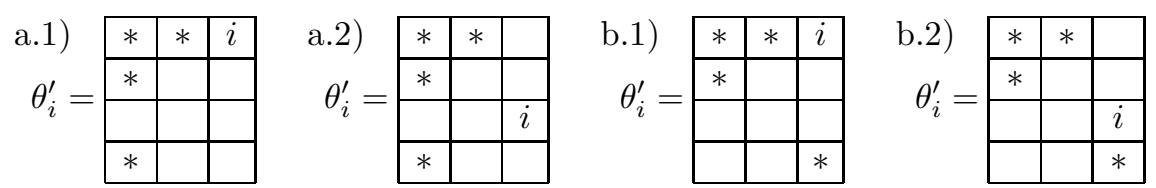

(1) First case: $i$ lies in the first row of $T$. We state this failure case:

(First failure case) If $i$ is in the first row of $T$ whereas the tableaux $\theta_{i-1}$ and $\tau[1, \ldots, i-1]$ do not coincide, then the algorithm fails.

We suppose that the failure case does not occur. Then we set $\theta_{i}=\theta_{i}^{\prime}$.

For example:
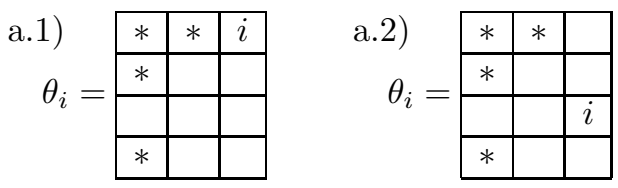

b.1), b.2): fail

(2) Second case: $i>1$, and $i$ belongs to the first column of $T$. We state:

(Second failure case) If $i>1$ belongs to the first column of $T$ whereas $s_{i-1}>0$ and the tableaux $\theta_{i}^{\prime}$ and $\tau[1, \ldots, i]$ coincide, then the algorithm fails.

We suppose that this failure case does not occur. Then there are nonempty boxes among the last $r-1$ boxes of the $\left(s_{i-1}+1\right)$-th column of $\theta_{i}^{\prime}$. Choose the entry in the upper row and push it to the first column. Let $\theta_{i}$ be the tableau so-obtained.

For example:

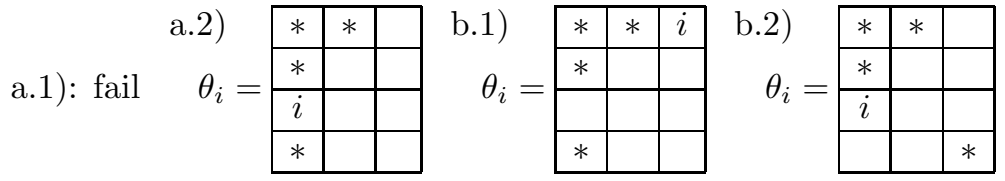

In each case, properties (h-a), (h-b), (h-c) are easily satisfied by $\theta_{i}$.

If no failure case occurs while $i$ runs over $\{1, \ldots, n\}$, then we get a final tableau $\theta_{n}$ with entries $1, \ldots, n$. According to (h-a) and (h-b) we have $\theta_{n}=\tau$. We say that the pair $(\tau, T)$ is constructible.

Example 7. (a) Suppose

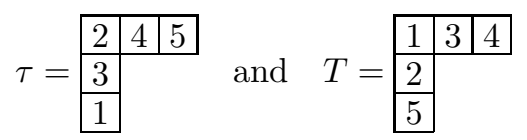

We get successively
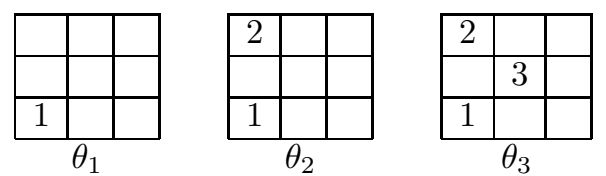

There is a failure of first type at the fourth step, since 4 belongs to the first row of $T$ whereas $\theta_{3}$ and $\tau[1, \ldots, 3]$ do not coincide.

(b) Suppose

$$
\tau=\begin{array}{|l|l|l|l|l|l|}
\hline 1 & 3 & 4 \\
& & \text { and } \quad T= & 2 & 5 \\
\hline 2 &
\end{array}
$$



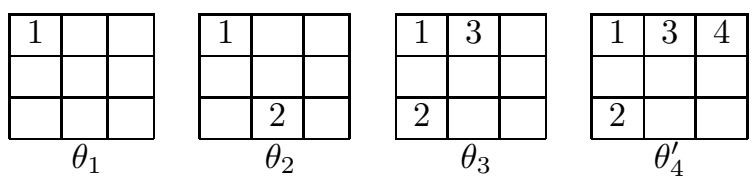

There is a failure of second type at the fourth step since 4 belongs to the first column of $T$ whereas the tableaux $\theta_{4}^{\prime}$ and $\tau[1, \ldots, 4]$ coincide.

(c) Suppose now

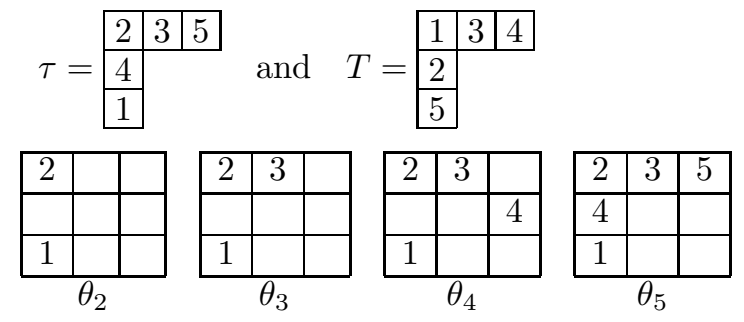
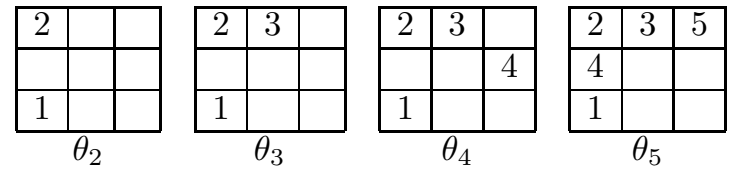

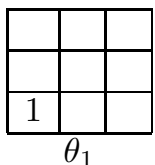

The pair $(\tau, T)$ is constructible.

We show the following result.

Theorem 28. Let $(\tau, T) \in \mathbf{Y}_{\mathrm{hk}}$. Then, we have $\tau \in T$ if and only if the pair $(\tau, T)$ is constructible.

Proof. Let $\mathbf{A}_{\mathrm{hk}}$ be the set introduced in section 5.1. According to Theorem 14, it is sufficient to show that $(\tau, T)$ is constructible if and only if $(\tau, T) \in \mathbf{A}_{\mathrm{hk}}$. Let $a_{1}=1<a_{2}<\ldots<a_{s}$ (resp. $a_{1}^{\prime}<a_{2}^{\prime}<\ldots<a_{s}^{\prime}$ ) denote the entries of the first row of $T$ (resp. of $\tau$ ). It is easy to see that, if a failure of first type occurs in $i$, then $i \in\left\{a_{2}, \ldots, a_{s}\right\}$, whereas if the failure is of second type, then $i \in\left\{a_{2}^{\prime}, \ldots, a_{s}^{\prime}\right\}$.

A first type failure occurs at the $i$-th step for $i=a_{q}(q \geq 2)$ if and only if $\theta_{i-1}$ and $\tau[1, \ldots, i-1]$ do not coincide. By $(\mathrm{h}-\mathrm{b})$, this is equivalent to the relation $a_{q-1}^{\prime}>i-1$. Equivalently, $a_{q-1}^{\prime} \geq a_{q}$.

Suppose $i=a_{q}^{\prime}$ with $q \geq 2$. As the tableau $\theta_{i-1}$ satisfies condition (h-b), we have $a_{q-1} \leq i-1$. There is a second type failure at the step $i$ if and only if $i$ is in the first column of $T$ whereas $\theta_{i-1}$ and $\tau[1, \ldots, i-1]$ coincide. Equivalently, $i \notin\left\{a_{2}, \ldots, a_{q}\right\}$ and $i-1<a_{q}$. This is equivalent to have $a_{q}^{\prime}=i<a_{q}$.

We get: the algorithm fails if and only if there is $q \in\{2, \ldots, s\}$ such that $a_{q-1}^{\prime} \geq a_{q}$ or $a_{q}>a_{q}^{\prime}$. This is equivalent to: $(\tau, T) \notin \mathbf{A}_{\mathrm{hk}}$. The proof is then complete.

\section{Connection to the PRoblem of intersections of COMPONENTS in CODIMENSION ONE IN THE TWO-ROW CASE}

In the last two sections of this article, we study the pairs of irreducible components $\mathcal{K}^{T}, \mathcal{K}^{S} \subset \mathcal{B}_{u}$ which intersect in codimension one, i.e. $\operatorname{codim}_{\mathcal{K}^{T}} \mathcal{K}^{T} \cap \mathcal{K}^{S}=1$ (recall that $\mathcal{B}_{u}$ is equidimensional, cf. 1.1). The motivation for investigating this question is a conjecture by D. Kazhdan and G. Lusztig (cf. [6, §6.3]) where these intersections of codimension one play a crucial role. The description of pairs of components $\mathcal{K}^{T}, \mathcal{K}^{S} \subset \mathcal{B}_{u}$ having an intersection in codimension one as well as the proof that they accord to Kazhdan-Lusztig conjecture have been provided in the hook, two-row and two-column cases and, up to now, only in these three cases.

Our purpose is to relate this question to the questions involved in the previous sections. Namely, still assuming that $T, S$ are of hook, two-row or two-column type, 
we will show that $\operatorname{codim}_{\mathcal{K}^{T}} \mathcal{K}^{T} \cap \mathcal{K}^{S}=1$ implies $(S \in T$ or $T \in S)$ : this is Theorem 41 of the next section.

In the present section, to begin with, we only deal with the two-row case. The pairs of components intersecting in codimension one have already been characterized in the two-row case (see [5] or [21]). Here, we provide a new characterization:

Theorem 29. Let $u \in \operatorname{End}(V)$ be a nilpotent endomorphism of two-row type. Let $\mathcal{K}^{T}, \mathcal{K}^{S} \subset \mathcal{B}_{u}$ be two components associated to the standard tableaux $T, S$. Then, the following conditions are equivalent:

(i) $\operatorname{codim}_{\mathcal{K}^{T}} \mathcal{K}^{T} \cap \mathcal{K}^{S}=1$;

(ii) $(S \in T$ or $T \in S)$ and all the entries of the first row of $T$ but one lie in the first row of $S$.

The previous characterization in 5 involves the combinatorics of cup-diagrams which also arises in the theory of representations of the Temperley-Lieb algebra (see [20]). We recall the definition of cup-diagrams and this characterization first.

Implicitly, 5] involves a procedure introduced by D. Vogan [19]. Vogan's transformation $\mathcal{T}_{\alpha, \beta}$ depends on two adjacent simple roots $\alpha, \beta$ of a given irreducible root system, the transformation $\mathcal{T}_{\alpha, \beta}$ is defined on elements of the Weyl group $w \in W$ such that $w^{-1}(\beta)<0$ and $w^{-1}(\alpha) \nless 0$. In type $A$, this transformation on the Weyl group (which is then the symmetric group) has a translation in terms of standard tableaux by the means of the Robinson-Schensted algorithm. See [9, §5] for more details. Here we recall only the procedure induced on tableaux.

Then, we show the above theorem, by connecting cup-diagrams and Vogan's procedure to the combinatorics involved in the previous sections.

7.1. The combinatorics of cup-diagrams and meanders. Let $T$ be a standard tableau with two rows. Let $n=|T|$. Let $r \geq s$ be the lengths of its two rows. Let $1=a_{1}<\ldots<a_{r}$ (resp. $b_{1}<\ldots<b_{s}$ ) be the entries in the first (resp. second) row of $T$. Let us define a sequence $a_{1}^{*}, \ldots, a_{s}^{*}$ by induction. Put $a_{1}^{*}=b_{1}-1$, and having defined $a_{1}^{*}, \ldots, a_{q-1}^{*}$ for $q \leq s$, put

$$
a_{q}^{*}=\max \left\{x \in\left\{a_{1}, \ldots, a_{r}\right\} \backslash\left\{a_{1}^{*}, \ldots, a_{q-1}^{*}\right\}: x<b_{q}\right\} .
$$

Note that this definition interprets in terms of parenthesis diagrams (cf. [20, $\S 2])$. We construct a word of $n$ letters in the alphabet $\{\bullet,()$,$\} . First put closing$ parentheses ")" at the positions number $b_{1}, \ldots, b_{s}$. Next, consider the letters ")" from left to right, for each one put its corresponding opening parenthesis " (" in the rightmost non-assigned letter on its left. Complete the word with $n-2 s$ letters "•" in the remaining places. The position of the "(" corresponding to the ")" at the position $b_{q}$ gives the number $a_{q}^{*}$.

Following [5], the cup-diagram of $T$ is the graph with $n$ points numbered by $1,2, \ldots, n$, displayed along a horizontal line, and with an arc connecting $a_{q}^{*}$ to $b_{q}$ for each $q=1, \ldots, s$. We say that the $a_{q}^{*}$ 's are the left end points, the $b_{q}$ 's are the right end points, and the remaining points are called fixed points.

Example 8. Let $T=$\begin{tabular}{|l|l|l|l|l|}
\hline 1 & 2 & 4 & 6 & 7 \\
\hline 3 & 5 & 8 & 9 & . The corresponding parenthesis diagram is
\end{tabular} "• ()()$(())$ ". The corresponding cup-diagram is

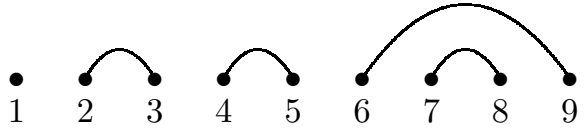


Let $T, S$ be two standard tableaux of same shape with two rows. Following [10], the meander $M_{T, S}$ is the graph obtained as follows. We draw the cup-diagrams of $T$ and $S$ on the same line of points $1, \ldots, n$, the arcs of the cup-diagram of $T$ being drawn upward and the arcs of the cup-diagram of $S$ being drawn downward.

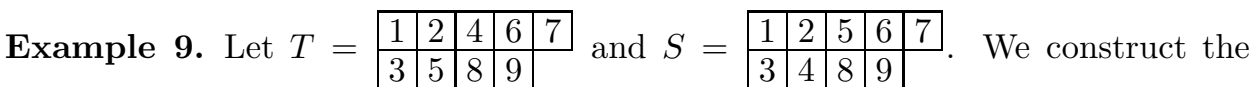
following meander $M_{T, S}$ :

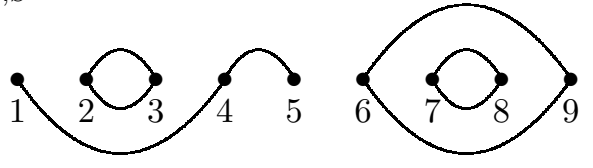

Following [10, §5] we introduce some terminology. A connected subset of arcs can be open or closed. We call loop a closed subset of arcs. We call interval an open subset of arcs. The number of arcs in a path is called its length. Note that the length of a loop is always even. The meander $M_{T, S}$ is said to be even if all of its intervals are even. It is said to be odd, otherwise. For example in the meander above, there are three loops and one interval of length 2 . The meander is even.

The following theorem is a consequence of [5, Theorems 7.3 and 7.4] and the results in [20, as it is explained in [10, §5.5].

Theorem 30. Let $u \in \operatorname{End}(V)$ be a nilpotent endomorphism of two-row type. Let $\mathcal{K}^{T}, \mathcal{K}^{S} \subset \mathcal{B}_{u}$ be two components associated to the standard tableaux $T, S$. Then, we have $\mathcal{K}^{T} \cap \mathcal{K}^{S} \neq \emptyset$ if and only if $M_{T, S}$ is even. Moreover, in this case, $\operatorname{dim} \mathcal{K}^{T} \cap \mathcal{K}^{S}$ is the number of loops of $M_{T, S}$.

If $r \geq s$ are the lengths of the Jordan blocks of $u$, then by 1.1, we have $\operatorname{dim} \mathcal{K}^{T}=$ $\operatorname{dim} \mathcal{B}_{u}=s$. It follows:

Corollary 31. Let $r \geq s$ be the lengths of the rows of $\operatorname{sh}(T)=\operatorname{sh}(S)$. Then, $\operatorname{codim}_{\mathcal{K}^{T}} \mathcal{K}^{T} \cap \mathcal{K}^{S}=1$ if and only if $M_{T, S}$ is an even meander with $s-1$ loops.

Example 10. (a) For $T, S$ as in Example 9, the meander $M_{T, S}$ is even and has 3 loops. It results $\operatorname{codim}_{\mathcal{K}^{T}} \mathcal{K}^{T} \cap \mathcal{K}^{S}=1$.

(b) In addition, let $R=$\begin{tabular}{|l|l|l|l|l}
\hline 1 & 2 & 3 & 4 & 7 \\
\hline 5 & 6 & 8 & 9
\end{tabular} . We see that the meander $M_{T, R}$ is even with 3 loops, hence $\operatorname{codim}_{\mathcal{K}^{T}} \mathcal{K}^{T} \cap \mathcal{K}^{R}=1$. The meander $M_{S, R}$ is even, hence $\mathcal{K}^{S}, \mathcal{K}^{R}$ have a nonempty intersection, but $M_{S, R}$ but has only 2 loops, hence this intersection has dimension two.

Definition of the set $\mathbf{M}_{2-\mathrm{r}}$. Let $T, S$ be standard tableaux of same shape with two rows of lengths $r \geq s$. Say $(S, T) \in \mathbf{M}_{2-\mathrm{r}}$ if the meander $M_{T, S}$ is even and has $s-1$ loops.

7.2. Vogan's $\mathcal{T}_{\alpha, \beta}$ procedure. We take the notation of $[9, \S 5]$. The set of the simple roots for the type $A_{n-1}$ is denoted by $\Pi=\left\{\alpha_{i}: i=1, \ldots, n-1\right\}$ with $\alpha_{i}=(i, i+1)$. Let $T$ be a standard tableau with $|T|=n$. For $i \in\{1, \ldots, n\}$, we denote by $r_{T}(i)$ the number of the row of $T$ containing $i$ (rows being numbered from top to bottom). We say that $i \in\{1, \ldots, n-1\}$ is a descent of $T$ if $r_{T}(i)<r_{T}(i+1)$.

We define the set $\mathcal{D}_{\alpha, \beta}$ for any two adjacent simple roots $\alpha, \beta$. Let $i=1, \ldots, n-2$. Define $\mathcal{D}_{\alpha_{i}, \alpha_{i+1}}$ as the set of standard tableaux $T$ with $|T|=n$ for which $i+1$ is a 
descent, but not $i$. Define $\mathcal{D}_{\alpha_{i+1}, \alpha_{i}}$ as the set of standard tableaux $T$ with $|T|=n$ for which $i$ is a descent, but not $i+1$.

Let $T \in \mathcal{D}_{\alpha_{i}, \alpha_{i+1}}$. We define the standard tableau $\mathcal{T}_{\alpha_{i}, \alpha_{i+1}}(T)$ as follows.

(a) If $r_{T}(i)<r_{T}(i+2)$, then $\mathcal{T}_{\alpha_{i}, \alpha_{i+1}}(T)$ is obtained from $T$ by interchanging $i+1$ and $i+2$.

(b) If $r_{T}(i) \geq r_{T}(i+2)$, then $\mathcal{T}_{\alpha_{i}, \alpha_{i+1}}(T)$ is obtained from $T$ by interchanging $i$ and $i+1$.

Thus $\mathcal{T}_{\alpha_{i}, \alpha_{i+1}}$ is a bijection from $\mathcal{D}_{\alpha_{i}, \alpha_{i+1}}$ to $\mathcal{D}_{\alpha_{i+1}, \alpha_{i}}$, and its inverse bijection is denoted by $\mathcal{T}_{\alpha_{i+1}, \alpha_{i}}$ : we obtain $\mathcal{T}_{\alpha_{i+1}, \alpha_{i}}(S)$ from $S \in \mathcal{D}_{\alpha_{i+1}, \alpha_{i}}$ by interchanging either $i$ and $i+1$, or $i+1$ and $i+2$, depending on whether $r_{T}(i)<r_{T}(i+2)$, or $r_{T}(i) \geq r_{T}(i+2)$.

In addition, for $i=2, \ldots, n-1$, define $\mathcal{D}_{i}$ as the set of standard tableaux $T$ with $|T|=n$ such that $i, i+1$ are neither in the same row nor in the same column of $T$. For $T \in \mathcal{D}_{i}$, let $\mathcal{T}_{i}(T)$ be the tableau obtained from $T$ by switching $i, i+1$.

If $T$ has two rows, then, observe that we have $T \in \mathcal{D}_{\alpha_{i}, \alpha_{i+1}}$ if and only if $i+1$ is a descent of $T$. Likewise, we have $T \in \mathcal{D}_{\alpha_{i+1}, \alpha_{i}}$ if and only if $i$ is a descent of $T$. Definition of the sets $\mathbf{V}$ and $\mathbf{V}_{2-\mathrm{r}}$. We denote by $\mathbf{V}$ the set of pairs of standard tableaux obtained as follows

$$
\left(\mathcal{T}_{\alpha^{(j)}, \beta^{(j)}} \cdots \mathcal{T}_{\alpha^{(1)}, \beta^{(1)}}(T), \mathcal{T}_{\alpha^{(j)}, \beta^{(j)}} \cdots \mathcal{T}_{\alpha^{(1)}, \beta^{(1)}} \mathcal{T}_{i}(T)\right)
$$

where $\left(\alpha^{(k)}, \beta^{(k)}\right)_{k=1, \ldots, j}$ is a sequence (possibly empty) of pairs of adjacent simple roots, and $T \in \mathcal{D}_{i}$ is such that the tableaux $\mathcal{T}_{\alpha^{(k)}, \beta^{(k)}} \cdots \mathcal{T}_{\alpha^{(1)}, \beta^{(1)}}(T)$ and $\mathcal{T}_{\alpha^{(k)}, \beta^{(k)}} \cdots \mathcal{T}_{\alpha^{(1)}, \beta^{(1)}} \mathcal{T}_{i}(T)$ are well defined for every $k=1, \ldots, j$. We denote by $\mathbf{V}_{\text {2-r }}$ the subset of pairs $(S, T) \in \mathbf{V}$ which have two rows.

Example 11. Let $T, S, R$ be as in the previous example. Then we have

$$
\begin{gathered}
(T, S)=\left(T, \mathcal{T}_{4}(T)\right) \in \mathbf{V}_{2-\mathrm{r}}, \\
(T, R)=\left(\mathcal{T}_{\alpha_{4}, \alpha_{5}}(Q), \mathcal{T}_{\alpha_{4}, \alpha_{5}} \mathcal{T}_{3}(Q)\right) \in \mathbf{V}_{2-\mathrm{r}}
\end{gathered}
$$

where $Q=$\begin{tabular}{|l|l|l|l|l|}
\hline 1 & 2 & 4 & 5 & 7 \\
\hline 3 & 6 & 8 & 9 &
\end{tabular} .

7.3. A new characterization of the intersections in codimension one. Theorem 29 follows from the following

Theorem 32. Let T, S be two standard tableaux of same shape with two rows. The following conditions are equivalent:

(i) $(T, S) \in \mathbf{M}_{2-\mathrm{r}}$;

(ii) $(T, S) \in \mathbf{V}_{2-\mathrm{r}}$;

(iii) $(S \in T$ or $T \in S$ ) and every entry in the first row of $T$ but one lies in the first row of $S$.

The end of this section is devoted to the proof of Theorem 32. The proof is obtained by combining Lemmas 33, 34 and 35. The first step is the following

Lemma 33. $\mathbf{V}_{2-\mathrm{r}} \subset \mathbf{M}_{2-\mathrm{r}}$.

Proof. If $T \in \mathcal{D}_{i}$ and $S=\mathcal{T}_{i}(T)$, then the fact that $\operatorname{codim}_{\mathcal{K}^{T}} \mathcal{K}^{T} \cap \mathcal{K}^{S}=1$ follows from [11, §3.1] (it can be shown also by using Theorem 30).

Let $T, S \in \mathcal{D}_{\alpha_{i}, \alpha_{i+1}}$ and put $T^{\prime}=\mathcal{T}_{\alpha_{i}, \alpha_{i+1}}(T)$ and $S^{\prime}=\mathcal{T}_{\alpha_{i}, \alpha_{i+1}}(S)$. We show:

$$
(T, S) \in \mathbf{M}_{2-\mathrm{r}} \Leftrightarrow\left(T^{\prime}, S^{\prime}\right) \in \mathbf{M}_{2-\mathrm{r}} .
$$


To do this, let us describe the changes between the cup-diagrams of $T$ and $T^{\prime}$.

(a) Assume $r_{T}(i)<r_{T}(i+2)$. Then $i, i+1$ are in the first row of $T$ and $i+2$ is in the second row, and $T^{\prime}$ is obtained by switching $i+1, i+2$. As $i+1$ is a descent of $T$, there is an arc joining $(i+1, i+2)$ in the cup-diagram of $T$. Possibly an arc starts at $i$ until some $i_{1}>i+2$ :

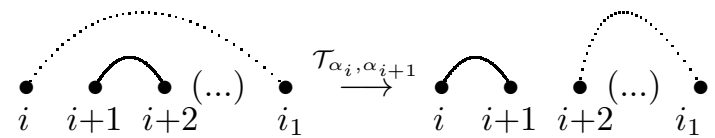

As represented in the picture, it is straightforward to check that the cup-diagram of $T^{\prime}$ is obtained by changing the arc joining $(i+1, i+2)$ into an $\operatorname{arc}(i, i+1)$, and changing the arc joining $\left(i, i_{1}\right)$, if exists, into an arc $\left(i+2, i_{1}\right)$.

(b) Assume $r_{T}(i) \geq r_{T}(i+2)$. Then $i+1$ is in the first row of $T$ and $i, i+2$ are in the second row, and $T^{\prime}$ is obtained by switching $i, i+1$. There is an arc joining $(i+1, i+2)$ in the cup-diagram of $T$. As $i$ is in the second row, there is an arc $\left(i_{0}, i\right)$ for some $i_{0}<i$ :

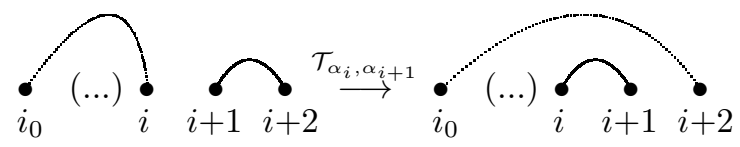

As in the picture, it is straightforward to see that the cup-diagram of $T^{\prime}$ is obtained by changing the arc joining $(i+1, i+2)$ into an arc $(i, i+1)$, and changing the arc joining $\left(i_{0}, i\right)$ into an arc $\left(i_{0}, i+2\right)$.

Now let us describe the changes between the meanders $M_{T, S}$ and $M_{T^{\prime}, S^{\prime}}$. In any case, there is an arc joining $(i+1, i+2)$ in the cup-diagrams of both $T$ and $S$, and possible $\operatorname{arcs}\left(i, i_{2}\right)$ and $\left(i, i_{2}^{\prime}\right)$ in both cup-diagrams (with either $i_{2}<i$ or $i_{2}>i+2$, and similarly $i_{2}^{\prime}<i$ or $i_{2}^{\prime}>i+2$, possibly $i_{2}=i_{2}^{\prime}$ ). The following picture illustrates the changes between the meanders $M_{T, S}$ and $M_{T^{\prime}, S^{\prime}}$ (the picture assumes for example $\left.i_{2}, i_{2}^{\prime}>i+2\right)$.

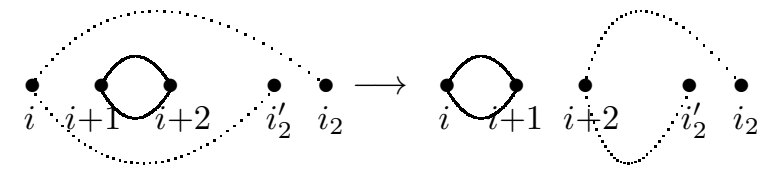

It is clear that the change $M_{T, S} \rightarrow M_{T^{\prime}, S^{\prime}}$ preserves the number of loops and the lengths of the intervals. Therefore, we get $(T, S) \in \mathbf{M}_{2-\mathrm{r}} \Leftrightarrow\left(T^{\prime}, S^{\prime}\right) \in \mathbf{M}_{2-\mathrm{r}}$. Then, the inclusion $\mathbf{V}_{2-\mathrm{r}} \subset \mathbf{M}_{2-\mathrm{r}}$ follows from the definition of $\mathbf{V}_{2-\mathrm{r}}$.

Next, we show the relation between Vogan's procedure and constructible pairs:

Lemma 34. Let $T, S$ be two standard tableaux of same shape with two rows. Assume $S \in T$, and that every entry in the first row of $T$ but one lies in the first row of $S$. Then we have $(T, S) \in \mathbf{V}_{2-\mathrm{r}}$.

Proof. Following section 6.1, we consider the algorithm of constructibility for the pair $(S, T)$. Let $\theta_{1}, \theta_{2}, \ldots$ be the tableaux obtained while applying the algorithm. As $S \in T$, by Theorem 21, the pair $(S, T)$ is constructible. Let $i$ be the minimal entry which has not the same place in $T$ and $S$. By definition of the algorithm, the tableau $\theta_{i-1}$ coincides with the subtableau $S[1, \ldots, i-1]$. In addition, as the 
algorithm does not fail at the $i$-th step, it follows that $i$ is in the first row of $T$ and in the second row of $S$. Thus, $\theta_{i}$ has the following form:

$$
\theta_{i}=\begin{array}{|l|l|l|}
\hline * * * * * & \\
\hline * * & & i \\
\hline
\end{array}
$$

The shape of this tableau is not a Young diagram since there are empty boxes on the left of $i$. As $(S, T)$ is constructible, the final tableau $\theta_{n}$ coincides with $S$, hence its shape is a Young diagram. Thus $i$ is pushed to the left, during the remaining steps. By definition of the algorithm, it implies that there is some $j>i$ in the second row of $T$. Take $j$ minimal.

We show $(S, T) \in \mathbf{V}_{2-\mathrm{r}}$ by induction on the pair $(n-i, j-i)$ for the lexicographical order. If $j=i+1$, then $T \in \mathcal{D}_{i}$. Moreover $i+1$ belongs to the first row of $S$, since the algorithm fails at the $(i+1)$-th step otherwise. Therefore, $S=\mathcal{T}_{i}(T)$ and we have $(T, S)=\left(T, \mathcal{T}_{i}(T)\right) \in \mathbf{V}_{2-\mathrm{r}}$.

Now, we suppose $j \geq i+2$. Then $i+1, \ldots, j-1$ are in the first row of $T$. By hypothesis $i$ is the only entry of the first row of $T$ which is not in the first row of $S$, hence $i+1, \ldots, j-1$ also belong to the first row of $S$. By definition of the algorithm, the tableau $\theta_{j-1}$ is as follows:

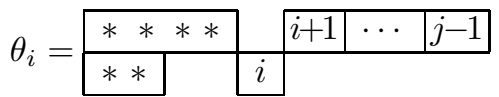

Since $j$ is in the second row of $T$, it is impossible that $j$ is in the first row of $S$, since there is a failure at the $j$-th step otherwise. Hence $j$ is in the second row of both $T, S$. It follows $T, S \in \mathcal{D}_{\alpha_{j-2}, \alpha_{j-1}}$.

Write $T^{\prime}=\mathcal{T}_{\alpha_{j-2}, \alpha_{j-1}}(T)$ and $S^{\prime}=\mathcal{T}_{\alpha_{j-2}, \alpha_{j-1}}(S)$. The tableau $T^{\prime}$ is obtained from $T$ by switching $j-1, j$. The tableau $S^{\prime}$ is obtained from $S$ by switching either $i, i+1$ or $j-1, j$ depending on whether $j=i+2$ or $j>i+2$. In the former case, $i+2$ is the minimal entry which has not the same place in $T^{\prime}, S^{\prime}$. In the latter case, $i$ is the minimal entry which has not the same place in $T^{\prime}, S^{\prime}$ and $j-1$ is the minimal entry, bigger than $i$, which lies in the second row of $T$. In both cases, the induction hypothesis applies, so that it is sufficient to show that $S^{\prime} \in T^{\prime}$.

To prove $S^{\prime} \in T^{\prime}$, we show that the pair $\left(S^{\prime}, T^{\prime}\right)$ is constructible. Let $\theta_{1}^{\prime}, \theta_{2}^{\prime}, \ldots$ be the tableaux obtained by applying the algorithm. Since $1, \ldots, i-1$ are in the same places in $T, S, T^{\prime}, S^{\prime}$, we have $\theta_{i-1}^{\prime}=\theta_{i-1}$.

(a) Suppose $j=i+2$. Then $i$ is in the first row of $T^{\prime}, S^{\prime}, i+1$ is in the second row of $T^{\prime}, S^{\prime}$ and $i+2$ is in the first row of $T^{\prime}$, in the second row of $S^{\prime}$. Then we have

$$
\theta_{i+2}^{\prime}=\begin{array}{|l|l|l|l|l|l|l|l|l|}
\hline * * * * * & * & * & * & * & i+1 & \\
\hline * * & i+1 & & i+2
\end{array} \text { whereas } \theta_{i+2}=\begin{array}{|l|l|l|l|}
\hline * * & i & & i+2 \\
\hline
\end{array}
$$

As $\theta_{i+2}, \theta_{i+2}^{\prime}$ have the same shape, and as $i+3, \ldots, n$ have the same places in $T, T^{\prime}$ on one hand, and in $S, S^{\prime}$ on the other hand, we get that $\left(S^{\prime}, T^{\prime}\right)$ is constructible if and only if $(S, T)$ is constructible.

(b) Suppose $j>i+2$. Then $i$ is in the first row of $T^{\prime}$, in the second row of $S^{\prime}$, $i+1, \ldots, j-2, j$ are in the first row of $T^{\prime}$ and $S^{\prime}, j-1$ is in the second row of $T^{\prime}$ and $S^{\prime}$. Then we have

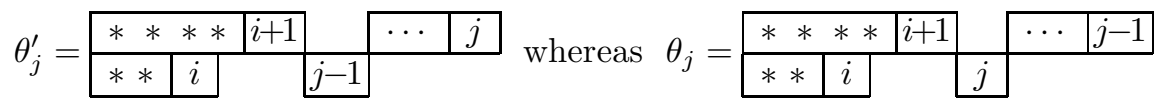


Thus $\theta_{j}, \theta_{j}^{\prime}$ have the same shape, moreover $j+1, \ldots, n$ have the same places in $T, T^{\prime}$ on one hand, and in $S, S^{\prime}$ on the other hand. Therefore, $\left(S^{\prime}, T^{\prime}\right)$ is constructible if and only if $(S, T)$ is constructible.

In both cases, we infer that $S^{\prime} \in T^{\prime}$. This argument completes the proof.

Finally, we prove:

Lemma 35. Let $(T, S) \in \mathbf{M}_{2-\mathrm{r}}$. Then, we have $(S \in T$ or $T \in S)$, and every entry in the first row of $T$ but one lies in the first row of $S$.

Proof. Let $r \geq s$ be the lengths of the rows of $\operatorname{sh}(T)=\operatorname{sh}(S)$, the common shape of $T, S$. First notice that the meander $M_{T, S}$ contains $s-1$ loops. For each loop, the rightmost point of the loop is a right end point of the cup-diagrams of both $T$ and $S$. It follows that the second rows of $T$ and $S$ have $s-1$ common entries. Equivalently, every entry in the first row of $T$ but one lies in the first row of $S$.

Say that the minimal entry which is not at the same place in $T, S$ lies in the first row of $T$ and in the second row of $S$. By [2, Lemma 2.3] and 4.2. the size of the second row of the diagram $Y_{j / i}^{T}$ is the number of $\operatorname{arcs}(a, b) \subset\{i+1, \ldots, j\}$ in the cup-diagram of $T$. On the other hand, the size of the second row of the diagram $Y_{j / i}(S)$ is the minimum between the number of entries among $i+1, \ldots, j$ in the first row of $S$ and the number of entries among $i+1, \ldots, j$ in the second row of $S$. Thus it is the minimum between the number of right end points $a \in\{i+1, \ldots, j\}$ in the cup-diagram of $S$ and the number of points $b \in\{i+1, \ldots, j\}$ which are not right end points in the cup-diagram of $S$.

We aim to show $S \preceq T$. By the previous observation, it is sufficient to show that, for any $0 \leq i<j \leq n$, the number of $\operatorname{arcs}(a, b) \subset\{i+1, \ldots, j\}$ in the cupdiagram of $T$ is less than or equal to the number of right end points $a \in\{i+1, \ldots, j\}$ and than the number of points $b \in\{i+1, \ldots, j\}$ which are not right end points in the cup-diagram of $S$. To do this, it is sufficient to show that, for any arc joining $(a, b)$ in the cup-diagram of $T,\{a, b\}$ contains exactly one right end-point of the cup-diagram of $S$.

The meander $M_{T, S}$ contains $2 s$ arcs and $s-1$ loops. It follows that at least $s-2$ loops have length 2. Moreover, if $M_{T, S}$ contains an (open) interval, then it contains $s-1$ loops of length 2 . If the arc $(a, b)$ of the cup-diagram of $T$ belongs to a loop of length two of the meander $M_{T, S}$, then it is also an arc in the cup-diagram of $S$. Then, $\{a, b\}$ contains $b$ as unique right end-point of the cup-diagram of $S$. Remove all the loops of length two of the meander $M_{T, S}$ and all the common fixed points of the cup-diagrams of $T$ and $S$, then there are two possible configurations for the remaining arcs:
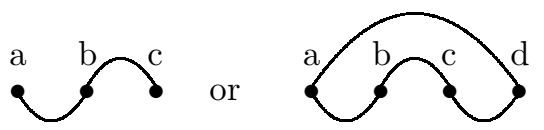

(recall that the arcs of the cup-diagram of $T$ are upward, the arcs of the cupdiagram of $S$ are downward). In each one of these two configurations, exactly one point among the end points of any arc of the cup-diagram of $T$ is a right end-point of the cup-diagram of $S$. This observation completes the proof. 


\section{Connection to THE PROBLEM OF INTERSECTIONS OF COMPONENTS IN CODIMENSION ONE}

In the previous section, we have shown in particular that for two components $\mathcal{K}^{T}, \mathcal{K}^{S} \subset \mathcal{B}_{u}$ of two-row type, we have:

$$
\operatorname{codim}_{\mathcal{K}^{T}} \mathcal{K}^{T} \cap \mathcal{K}^{S}=1 \Rightarrow(S \in T \text { or } T \in S) .
$$

In the present section, we show this implication also in the hook and two-column cases. In addition, we deduce some topological properties of the components.

8.1. On the intersections of codimension one in the three cases. To begin with we recall from 10 and 18 the description of the pairs of components intersecting in codimension one, for the hook and two-column cases, and we recall the already known property of irreducibility of the intersections in codimension one for the hook, two-row and two-column cases.

First, let $u \in \operatorname{End}(V)$ be nilpotent of hook type. Let $\mathcal{K}^{T}, \mathcal{K}^{S} \subset \mathcal{B}_{u}$ be the components associated to the standard tableaux $T, S$. Let $a_{1}=1<a_{2}<\ldots<a_{s}$ (resp. $a_{1}^{\prime}=1<a_{2}^{\prime}<\ldots<a_{s}^{\prime}$ ) be the entries in the first row of $T$ (resp. of $S$ ). By [18] (or Proposition 7 and Theorem[14above) the intersection $\mathcal{K}^{T} \cap \mathcal{K}^{S}$ is nonempty if and only if

$$
\max \left(a_{q-1}, a_{q-1}^{\prime}\right)<\min \left(a_{q}, a_{q}^{\prime}\right) \forall q=2, \ldots, s .
$$

Assume that the intersection $\mathcal{K}^{T} \cap \mathcal{K}^{S}$ is nonempty. Then, from [18, we have the following formula:

$$
\operatorname{codim}_{\mathcal{K}^{T}} \mathcal{K}^{T} \cap \mathcal{K}^{S}=\sum_{q=2}^{s}\left|a_{q}-a_{q}^{\prime}\right|
$$

and we can state:

Theorem 36. Let $u \in \operatorname{End}(V)$ be nilpotent of hook type. The intersection $\mathcal{K}^{T} \cap \mathcal{K}^{S}$ is nonempty of codimension one if and only if $T, S$ are obtained one from the other by switching two entries $i, i+1$ for some $i=2, \ldots, n-1$.

Let $\mathbf{V}_{\mathrm{hk}}$ denote the subset of pairs $(T, S) \in \mathbf{V}$ which are of hook type (cf. 7.2). It is easy to see that $\mathbf{V}_{\mathrm{hk}}$ is the set of pairs $\left(T, \mathcal{T}_{i}(T)\right)$ for $T \in \mathcal{D}_{i}$ of hook type and $i=2, \ldots, n-1$. Then, we get:

Corollary 37. Let $u \in \operatorname{End}(V)$ be nilpotent of hook type. We have $\operatorname{codim}_{\mathcal{K}^{T}} \mathcal{K}^{T} \cap$ $\mathcal{K}^{S}=1$ if and only if $(T, S) \in \mathbf{V}_{\mathrm{hk}}$.

Intersections in codimension one are closely connected in the two-row and twocolumn cases. Let $u \in \operatorname{End}(V)$ be nilpotent of two-column type. Let $\mathcal{K}^{T}, \mathcal{K}^{S} \subset \mathcal{B}_{u}$ be the components associated to the standard tableaux $T, S$. Let $T^{t}, S^{t}$ be the corresponding tableaux with two rows, obtained by transposition (see section 5.4). By [10, §5.4], we have:

Theorem 38. Let $u \in \operatorname{End}(V)$ be nilpotent of two-column type. Then, we have $\operatorname{codim}_{\mathcal{K}^{T}} \mathcal{K}^{T} \cap \mathcal{K}^{S}=1$ if and only if $\operatorname{codim}_{\mathcal{K}^{S^{t}}} \mathcal{K}^{T^{t}} \cap \mathcal{K}^{S^{t}}=1$.

As in section [7.2, let $\alpha, \beta \in\left\{\alpha_{1}, \ldots, \alpha_{n-1}\right\}$ be two consecutive simple roots. Then it is easy to see that, if $T$ is a standard tableau of two-column type, then $T \in \mathcal{D}_{\alpha, \beta}$ if and only if $T^{t} \in \mathcal{D}_{\beta, \alpha}$, and moreover in this case $\mathcal{T}_{\beta, \alpha}\left(T^{t}\right)=S^{t}$, where $S=\mathcal{T}_{\alpha, \beta}(T)$. Likewise, for $i \in\{1, \ldots, n-1\}$ we have $T \in \mathcal{D}_{i}$ if and only if $T^{t} \in \mathcal{D}_{i}$, and in this case $\mathcal{T}_{i}\left(T^{t}\right)=\left(\mathcal{T}_{i}(T)\right)^{t}$. Let $\mathbf{V}_{2 \text {-c }}$ denote the subset of pairs $(T, S) \in \mathbf{V}$ 
which have two columns. It follows that $(T, S) \in \mathbf{V}_{2-\mathrm{c}}$ if and only if $\left(T^{t}, S^{t}\right) \in \mathbf{V}_{2-\mathrm{r}}$. Combining this observation with Theorems 32 and 38 , we get:

Corollary 39. Let $u \in \operatorname{End}(V)$ be nilpotent of two-column type. Then, we have $\operatorname{codim}_{\mathcal{K}^{T}} \mathcal{K}^{T} \cap \mathcal{K}^{S}=1$ if and only if $(T, S) \in \mathbf{V}_{2-\mathrm{c}}$.

Thus it holds that, for $T, S$ a pair of standard tableaux of same shape of hook, two-row or two-column type, the components $\mathcal{K}^{T}$ and $\mathcal{K}^{S}$ intersect in codimension one if and only if $(T, S) \in \mathbf{V}$.

Finally, let us recall that the intersections in codimension one are irreducible in the three cases we consider here.

Theorem 40. Let $u \in \operatorname{End}(V)$ be nilpotent of hook, two-row or two-column type. Let $\mathcal{K}^{T}, \mathcal{K}^{S} \subset \mathcal{B}_{u}$ be the components associated to the standard tableaux $T, S$. If $\operatorname{codim}_{\mathcal{K}^{T}} \mathcal{K}^{T} \cap \mathcal{K}^{S}=1$, then $\mathcal{K}^{T} \cap \mathcal{K}^{S}$ is irreducible.

This result is proved in 18 for the hook case, in 5 for the two-row case and in 12 for the two-column case. Notice that the intersection $\mathcal{K}^{T} \cap \mathcal{K}^{S}$ can be reducible when $\operatorname{codim}_{\mathcal{K}^{T}} \mathcal{K}^{T} \cap \mathcal{K}^{S}>1$ (see [12]).

8.2. A necessary condition for an intersection in codimension one. The main result of this section is the following

Theorem 41. Let $u \in \operatorname{End}(V)$ be a nilpotent endomorphism of hook, two-row or two-column type. Let $\mathcal{K}^{T}, \mathcal{K}^{S} \subset \mathcal{B}_{u}$ be the components associated to the standard tableaux $T, S$. We have:

$$
\operatorname{codim}_{\mathcal{K}^{T}} \mathcal{K}^{T} \cap \mathcal{K}^{S}=1 \Rightarrow(S \in T \text { or } T \in S) .
$$

Proof. The result holds in the two-row case, by Theorem 29. Then, we deduce that the statement holds in the two-column case, using Proposition 18 and Theorem 38 It remains to show the result in the hook case. Suppose that $T, S$ are of hook type, and assume that $\operatorname{codim}_{\mathcal{K}^{T}} \mathcal{K}^{T} \cap \mathcal{K}^{S}=1$. By Theorem 36, the tableaux $T$ and $S$ are obtained one from the other by switching $i, i+1$ for some $i=2, \ldots, n-1$. Since both tableaux are standard, $i, i+1$ do not lie both in the first column or in the first row of $T$ (resp. of $S$ ). Thus $i$ is either in the first row of $T$ or in the first row of $S$. Say that it is in the first row of $T$. Then, applying Theorem 14 we easily obtain that $S \in T$.

8.3. Topological properties of the intersections in codimension one in the hook, two-row and two-column cases. We now establish some corollaries of Theorem 41. We consider two components $\mathcal{K}^{T}, \mathcal{K}^{S} \subset \mathcal{B}_{u}$ associated to standard tableaux $T, S$. Recall that they are obtained as the closures of the subsets $\mathcal{B}_{u}^{T}, \mathcal{B}_{u}^{S} \subset$ $\mathcal{B}_{u}$ (see 1.1).

Corollary 42. Let $u \in \operatorname{End}(V)$ be of hook, two-row or two-column type. If $\mathcal{K}^{T} \cap \mathcal{K}^{S}$ has codimension 1 in $\mathcal{K}^{T}$, then one of its open subsets $\mathcal{K}^{T} \cap \mathcal{B}_{u}^{S}$ and $\mathcal{B}_{u}^{T} \cap \mathcal{K}^{S}$ is nonempty and dense.

Proof. Using Lemma 3 and applying Theorem 41, we get that $\mathcal{B}_{u}^{T} \cap \mathcal{K}^{S}$ or $\mathcal{K}^{T} \cap \mathcal{B}_{u}^{S}$ is nonempty. Then it is dense because, by Theorem 40, the intersection $\mathcal{K}^{T} \cap \mathcal{K}^{S}$ is irreducible.

Let $\mathcal{K}^{R}$ be a third component associated to another standard tableau $R$. 
Proposition 43. Let $u \in \operatorname{End}(V)$ be of hook, two-row or two-column type. Suppose that $\operatorname{codim}_{\mathcal{K}^{T}} \mathcal{K}^{T} \cap \mathcal{K}^{S} \cap \mathcal{K}^{R}=1$, then two tableaux among $T, S, R$ coincide.

Proof. We define a total order on standard tableaux: let $i \in\{1, \ldots, n\}$ be minimal which has not the same place in $T, S$ and let $Y_{i}^{T}$ (resp. $Y_{i}^{S}$ ) be the shape of the subtableau of $T$ (resp. of $S$ ) of entries $1, \ldots, i$. We have either $Y_{i}^{T} \prec Y_{i}^{S}$ or $Y_{i}^{S} \prec Y_{i}^{T}$. Then, write $S<T$ if $Y_{i}^{S} \prec Y_{i}^{T}$. By Theorem 11, $S<T$ implies $T \notin S$.

Assume by contradiction that $T, S, R$ are pairwise distinct. We may assume $R<S<T$. Then, by Theorem 41, we have $R \in S, R \in T$ and $S \in T$. By Corollary 42, $\operatorname{codim}_{\mathcal{K}^{T}} \mathcal{K}^{T} \cap \mathcal{B}_{u}^{S}=1$. As $\mathcal{K}^{T} \cap \mathcal{K}^{S} \cap \mathcal{B}_{u}^{R}$ is a nonempty open subset of $\mathcal{K}^{T} \cap \mathcal{K}^{S} \cap \mathcal{K}^{R}$, we have $\operatorname{codim}_{\mathcal{K}^{T}} \mathcal{K}^{T} \cap \mathcal{K}^{S} \cap \mathcal{B}_{u}^{R}=1$. The sets $\mathcal{K}^{T} \cap \mathcal{B}_{u}^{S}$ and $\mathcal{K}^{T} \cap \mathcal{K}^{S} \cap \mathcal{B}_{u}^{R}$ are disjoint, locally closed subsets of $\mathcal{K}^{T} \cap \mathcal{K}^{S}$ and they have the same dimension as $\mathcal{K}^{T} \cap \mathcal{K}^{S}$. It contradicts the irreducibility of $\mathcal{K}^{T} \cap \mathcal{K}^{S}$.

8.4. A subset $\hat{\mathcal{K}}^{T}$ associated to generalized Spaltenstein's partitions. The subset $\mathcal{B}_{u}^{T} \subset \mathcal{B}_{u}$ is the set of $u$-stable flags $\left(V_{0}, \ldots, V_{n}\right) \in \mathcal{B}_{u}$ such that the Jordan shapes of the restrictions $u_{\mid V_{i}}$ of $u$ coincide with the shapes of the subtableaux $T[1, \ldots, i]$. The set $\mathcal{B}_{u}^{T}$ is thus defined by simple relations. Nevertheless, the difference between $\mathcal{B}_{u}^{T}$ and its closure, the component $\mathcal{K}^{T} \subset \mathcal{B}_{u}$, is big in general: one can see that, whenever $\mathcal{B}_{u}$ is reducible, it admits a component $\mathcal{K}^{T}$ with $\operatorname{codim}_{\mathcal{K}^{T}} \mathcal{K}^{T} \backslash \mathcal{B}_{u}^{T}=1$. We aim to construct a subset $\hat{\mathcal{K}}^{T} \subset \mathcal{K}^{T}$ bigger than $\mathcal{B}_{u}^{T}$, which is also defined by simple relations. To do this we rely on a generalization of the sets $\mathcal{B}_{u}^{T}$. Then we show that, in the hook, two-row and two-column cases, we have $\operatorname{codim}_{\mathcal{K}^{T}} \mathcal{K}^{T} \backslash \hat{\mathcal{K}}^{T} \geq 2$.

Recall a construction from 11. Let $\mathcal{R}_{n}$ denote the set of double sequences of integers $\left(i_{k}, j_{k}\right)_{k=0, \ldots, n}$ with $\left(i_{k}\right)_{k}$ weakly decreasing, $\left(j_{k}\right)_{k}$ weakly increasing, $0 \leq$ $i_{k} \leq j_{k} \leq n$ and $j_{k}-i_{k}=k$ for every $k$. Let $\rho=\left(i_{k}, j_{k}\right)_{k} \in \mathcal{R}_{n}$. Instead of considering the restrictions of $u$ to the subspaces of the flag, we consider the maximal chain of subquotients

$$
0=V_{j_{0}} / V_{i_{0}} \hookrightarrow V_{j_{1}} / V_{i_{1}} \hookrightarrow \ldots \hookrightarrow V_{j_{n}} / V_{i_{n}}=V .
$$

For $k=1, \ldots, n$ we consider the Young diagram $Y\left(u_{\mid V_{j_{k}} / V_{i_{k}}}\right)$ associated to the nilpotent $u_{\mid V_{j_{k}} / V_{i_{k}}} \in \operatorname{End}\left(V_{j_{k}} / V_{i_{k}}\right)$ induced by $u$. In addition, recall that $Y_{j_{k} / i_{k}}^{T}$ is defined in 4.2. We denote by $\mathcal{B}_{u, \rho}^{T}$ the set of $u$-stable flags $\left(V_{0}, \ldots, V_{n}\right)$ such that $Y\left(u_{\mid V_{j_{k}} / V_{i_{k}}}\right)=Y_{j_{k} / i_{k}}^{T}$ for every $k$. We get a partition $\mathcal{B}_{u}=\bigsqcup_{T} \mathcal{B}_{u, \rho}^{T}$ parameterized by standard tableaux of shape $Y(u)$. By 4.2 , the set $\mathcal{B}_{u, \rho}^{T}$ is a dense open subset of the component $\mathcal{K}^{T}$.

Proposition 44. Let $u \in \operatorname{End}(V)$ be of hook, two-row or two-column type. If $\mathcal{K}^{T} \cap \mathcal{K}^{S}$ has codimension 1 in $\mathcal{K}^{T}$, then there is $\rho \in \mathcal{R}_{n}$ such that one of the open subsets $\mathcal{B}_{u, \rho}^{T} \cap \mathcal{B}_{u}^{S}$ and $\mathcal{B}_{u}^{T} \cap \mathcal{B}_{u, \rho}^{S}$ of $\mathcal{K}^{T} \cap \mathcal{K}^{S}$ is nonempty and dense.

We need the following technical

Lemma 45. Let $T, S$ be two standard tableaux of common shape of hook, two-row or two-column type. Suppose $T \neq S$. Then there are $i<j$ such that $Y_{j / i}^{T} \prec Y_{j / i}^{S}$.

Proof of Lemma 45. Let $n=|T|=|S|$. Consider first the hook case. Let $L_{T}$ be the set of elements $i \in\{1, \ldots, n-1\}$ such that $i$ is situated on the left of $i+1$ in the tableau $T$. Observe that $i \in L_{T}$ if and only if $i+1$ is in the first row of $T$. Hence $\# L_{T}+1$ is the length of the first row of $T$. Suppose that we have $Y_{j / i}^{S} \preceq Y_{j / i}^{T}$ for 
any $i<j$. Then in particular $Y_{i+1 / i-1}^{S} \preceq Y_{i+1 / i-1}^{T}$ for $i \in L_{S}$ implies $L_{S} \subset L_{T}$. Thus $L_{S}=L_{T}$. It follows that the first rows of $T$ and $S$ coincide, hence $T=S$.

Suppose that $T, S$ have two rows of lengths $r \geq s$. Argue by induction on $n$, with immediate initialization for $n=1$. Assume that the property holds until in $n-1$. Suppose $Y_{j / i}^{S} \preceq Y_{j / i}^{T}$ for any $i<j$. We distinguish three cases.

(a) Suppose that there is $i<n$ such that the subtableau $T[1, \ldots, i]$ has a rectangular shape with two rows of length $i / 2$. As $Y_{i / 0}^{S} \preceq Y_{i / 0}^{T}$, the subtableaux $T[1, \ldots, i]$ and $S[1, \ldots, i]$ have the same shape, and the skew subtableaux $T[i+1, \ldots, n]$ and $S[i+$ $1, \ldots, n]$ have the same shape, which is a Young diagram. By induction hypothesis, we get $T[1, \ldots, i]=S[1, \ldots, i]$ and $T[i+1, \ldots, n]=S[i+1, \ldots, n]$, hence $T=S$.

(b) Suppose that $r=s=n / 2$. Then $n$ has the same place in $T, S$. Let $T^{\prime}=$ $T[1, \ldots, n-1]$ and $S^{\prime}=S[1, \ldots, n-1]$. By induction hypothesis, we get $T^{\prime}=S^{\prime}$. It follows $T=S$.

(c) Suppose that there is no $i \leq n$ for which $T[1, \ldots, i]$ is rectangular. Let $T^{\prime}$ (resp. $S^{\prime}$ ) be the rectification by jeu de taquin of the skew subtableau $T[2, \ldots, n]$ (resp. $S[2, \ldots, n])$. Then, as observed in $5.2(\mathrm{c}), T^{\prime}$ has two rows of lengths $(r-1, s)$. Since $Y_{n / 1}^{S} \preceq Y_{n / 1}^{T}$, the tableaux $T^{\prime}, S^{\prime}$ have the same shape. By induction hypothesis, we get $T^{\prime}=S^{\prime}$. It follows $T=S$. The argument in the two-row case is now complete.

Finally, suppose that $T, S$ have a common shape of two-column type and assume $T \neq S$. Let $T^{t}, S^{t}$ be the transposed tableaux with two rows (see 5.4). Then there are $i<j$ such that $Y_{j / i}^{S^{t}} \prec Y_{j / i}^{T^{t}}$. It follows $Y_{j / i}^{T} \prec Y_{j / i}^{S}$.

Proof of Proposition 44. By Corollary 42, we may assume $\mathcal{K}^{T} \cap \mathcal{B}_{u}^{S} \neq \emptyset$. By Lemma 45. there are $i<j$ such that $Y_{j / i}^{T} \prec Y_{j / i}^{S}$. We can find $\rho=\left(i_{k}, j_{k}\right)_{k=1, \ldots, n} \in \mathcal{R}_{n}$ such that $i_{k}=i$ and $j_{k}=j$ for $k=j-i$. Then, by Lemma 10 and the definition of $\mathcal{B}_{u, \rho}^{S}$, we have $\mathcal{K}^{T} \cap \mathcal{B}_{u, \rho}^{S}=\emptyset$. On the other hand, as the $\mathcal{B}_{u, \rho}^{R}$ 's form a partition of $\mathcal{B}_{u}$ into locally closed subsets, there is $R$ standard such that $\mathcal{K}^{T} \cap \mathcal{B}_{u}^{S} \cap \mathcal{B}_{u, \rho}^{R}$ is dense in $\mathcal{K}^{T} \cap \mathcal{B}_{u}^{S}$. It implies $\operatorname{codim}_{\mathcal{K}^{T}} \mathcal{K}^{T} \cap \mathcal{K}^{S} \cap \mathcal{K}^{R}=1$, hence by Proposition 43 we have $R \in\{T, S\}$. Moreover, as $\mathcal{K}^{T} \cap \mathcal{B}_{u, \rho}^{S}=\emptyset$, necessarily $R=T$. Therefore, $\mathcal{B}_{u, \rho}^{T} \cap \mathcal{B}_{u}^{S}$ is nonempty. By Theorem 40, it is dense in $\mathcal{K}^{T} \cap \mathcal{K}^{S}$.

Remark 4. Observe that the lemma is not necessarily true, when $T, S$ are not supposed of hook, two-row or two-column type. Indeed, consider

$$
T=\begin{array}{|l|l|l}
\hline 1 & 2 & 5 \\
\hline 3 & 4 &
\end{array} \quad S=\begin{array}{|l|l|l|}
\hline 1 & 2 & 5 \\
\hline 3 & & 6 \\
\hline 4 &
\end{array}
$$

Then we have $Y_{j / i}^{S} \preceq Y_{j / i}^{T}$ for any $i<j$, although $T \neq S$. One can also see that for any $\rho=\left(i_{k}, j_{k}\right)_{k=1, \ldots, 6} \in \mathcal{R}_{6}$ we have $Y_{j_{5} / i_{5}}^{S} \prec Y_{j_{5} / i_{5}}^{T}$. Then, by Lemma 10] and the definition of $\mathcal{B}_{u, \rho}^{T}$, we have $\mathcal{B}_{u, \rho}^{T} \cap \mathcal{K}^{S}=\emptyset$. Hence $\mathcal{B}_{u, \rho}^{T} \cap \mathcal{B}_{u}^{S}=\emptyset$. However, we can compute that the components $\mathcal{K}^{T}$ and $\mathcal{K}^{S}$ have a nonempty intersection which has codimension one. Therefore, the proposition does not hold in this case. Adding boxes to $T$ and $S$, we can show likewise that the proposition does not hold whenever the Jordan shape of $u$ contains $\stackrel{\square}{\square} \square$ as a subdiagram.

Finally, we define

$$
\hat{\mathcal{K}}^{T}=\bigcup_{\rho \in \mathcal{R}_{n}} \mathcal{B}_{u, \rho}^{T}
$$


This is an open subset of the component $\mathcal{K}^{T}$. We show:

Theorem 46. Assume that $T$ is of hook, two-row or two-column type. Then, we have $\operatorname{codim}_{\mathcal{K}^{T}} \mathcal{K}^{T} \backslash \hat{\mathcal{K}}^{T} \geq 2$.

Proof. Arguing by contradiction, we assume $\operatorname{codim}_{\mathcal{K}^{T}} \mathcal{K}^{T} \backslash \hat{\mathcal{K}}^{T}=1$. Then there is $S$ standard such that $\operatorname{codim}_{\mathcal{K}^{T}}\left(\mathcal{K}^{T} \backslash \hat{\mathcal{K}}^{T}\right) \cap \mathcal{B}_{u}^{S}=1$. In particular $\operatorname{codim}_{\mathcal{K}^{T}} \mathcal{K}^{T} \cap \mathcal{B}_{u}^{S}=$ 1. As $\mathcal{K}^{T} \cap \mathcal{K}^{S}$ is irreducible and $\mathcal{B}_{u}^{S} \cap \mathcal{B}_{u}^{T}=\emptyset$, we have $\mathcal{K}^{S} \cap \mathcal{B}_{u}^{T}=\emptyset$. By Proposition 44, there is $\rho \in \mathcal{R}_{n}$ such that $\operatorname{codim}_{\mathcal{K}^{T}} \mathcal{B}_{u, \rho}^{T} \cap \mathcal{B}_{u}^{S}=1$. We have thus $\operatorname{codim}_{\mathcal{K}^{T}}\left(\mathcal{K}^{T} \backslash \hat{\mathcal{K}}^{T}\right) \cap \mathcal{B}_{u}^{S}=1$ and $\operatorname{codim}_{\mathcal{K}^{T}} \hat{\mathcal{K}}^{T} \cap \mathcal{B}_{u}^{S}=1$. However, the set $\mathcal{K}^{T} \cap \mathcal{B}_{u}^{S}$ is irreducible, since it is open in $\mathcal{K}^{T} \cap \mathcal{K}^{S}$. This brings a contradiction.

Remark 5. Let T, $S$ be as in Remark 4 . Then we have $\mathcal{B}_{u, \rho}^{T} \cap \mathcal{K}^{S}=\emptyset$ for any $\rho \in \mathcal{R}_{n}$, hence $\mathcal{K}^{T} \cap \mathcal{K}^{S} \subset \mathcal{K}^{T} \backslash \hat{\mathcal{K}}^{T}$. Since the components $\mathcal{K}^{T}, \mathcal{K}^{S}$ have an intersection in codimension one, it follows codim $\mathcal{K}^{T} \mathcal{K}^{T} \backslash \hat{\mathcal{K}}^{T}=1$. The theorem does not hold in this case. Adding boxes to $T, S$, we show likewise that the theorem does not hold whenever the Jordan shape of $u$ contains

\section{INDEX OF THE NOTATION}

$1 \mathcal{B}, \mathcal{B}_{u}$

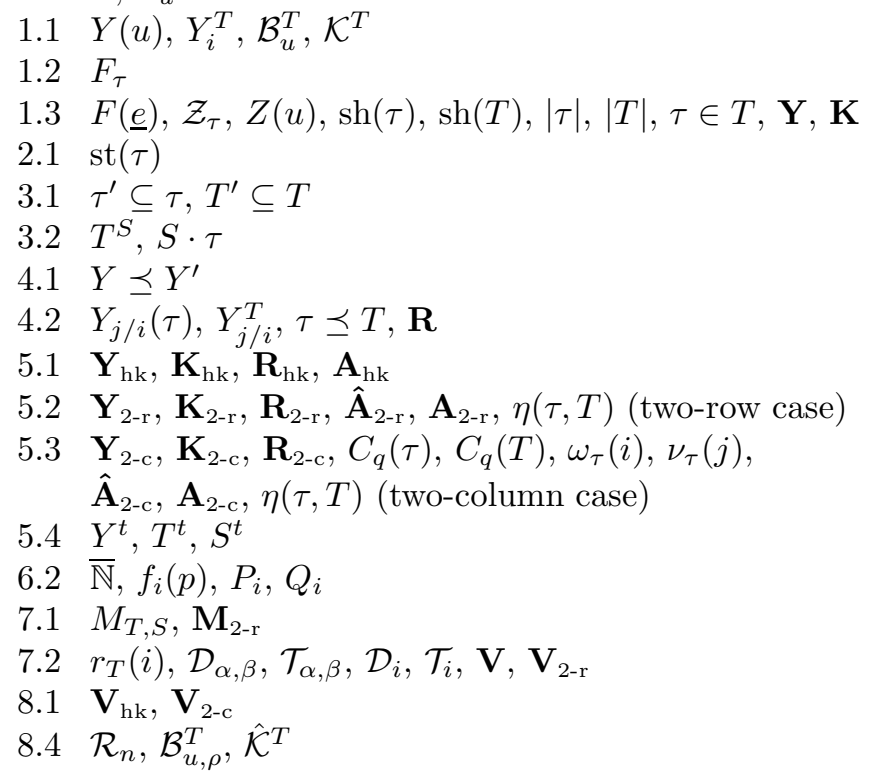

\section{REFERENCES}

[1] L. Fresse, Betti numbers of Springer fibers in type A, J. Algebra 322 (2009) 2566-2579.

[2] L. Fresse, Singular components of the Springer fiber in the two-column case, Ann. Inst. Fourier (Grenoble) 59 (2009) 2429-2444.

[3] L. Fresse, A. Melnikov, Some characterizations of singular components of Springer fibers in the two-column case, arXiv:0909.4008

[4] W. Fulton, Young Tableaux, with applications to representation theory and geometry, Cambridge University Press, 1997.

[5] F.Y.C. Fung, On the topology of components of some Springer fibers and their relation to Kazhdan-Lusztig theory, Adv. Math. 178 (2003) 244-276. 
[6] D. Kazhdan, G. Lusztig, Representations of Coxeter groups and Hecke algebras, Invent. Math. 53 (1979) 165-184.

[7] D. Kazhdan, G. Lusztig, A topological approach to Springer's representations, Adv. Math. 38 (1980) 222-228.

[8] M.A.A. van Leeuwen, Flag varieties and interpretations of Young tableau algorithms, J. Algebra 224 (2000) 397-426.

[9] A. Melnikov, On orbital variety closures in $s_{n}$ III. Geometric properties, J. Algebra 305 (2006) 68-97.

[10] A. Melnikov, B-orbits of nilpotent order 2 and link patterns, arXiv:math/07 03371v2.

[11] A. Melnikov, N.G.J. Pagnon, On intersections of orbital varieties and components of Springer fiber, J. Algebra 298 (2006) 1-14.

[12] A. Melnikov, N.G.J. Pagnon, Codimension one intersections of the components of a Springer fiber for the two-column case, Indag. Math., N.S., 20 (2009).

[13] N. Spaltenstein, The fixed point set of a unipotent transformation on the flag manifold, Indag. Math. 38 (1976) 452-456.

[14] N. Spaltenstein, Classes Unipotentes et Sous-Groupes de Borel, Lecture Notes in Mathematics 946, Springer-Verlag, Berlin-New York, 1982.

[15] T.A. Springer, The unipotent variety of a semisimple group, Proc. of the Bombay Colloqu. in Algebraic Geometry, ed. S. Abhyankar, London, Oxford Univ. Press (1969) 373-391.

[16] T.A. Springer, Trigonometric sums, Green functions of finite groups, and representations of Weyl groups, Invent. Math. 36 (1976) 173-207.

[17] R. Steinberg, On the desingularization of the unipotent variety, Invent. Math. 36 (1976) 209-224.

[18] J.A. Vargas, Fixed points under the action of unipotent elements of $S L(n)$ in the flag variety, Bol. Soc. Mat. Mexicana 24 (1979) 1-14.

[19] D.A. Vogan, A generalized $\tau$-invariant for the primitive spectrum of a semisimple Lie algebra, Math. Ann. 242 (1979) 209-224.

[20] B.W. Westbury, The representation theory of the Temperley-Lieb algebras, Math. Z. 219 (1995) 539-565.

[21] J. Wolper, Some intersection properties of the fibres of Springer's resolution, Proc. Am. Math. Soc. 91 (1984) 182-188.

Department of Mathematics, the Weizmann Institute of Science, Rehovot 76100, ISRAEL

E-mail address: lucas.fresse@weizmann.ac.il 